San Jose State University

SJSU ScholarWorks

Master's Projects

Master's Theses and Graduate Research

Fall 2021

\title{
Diversity Management Programs: California Local Governments' Approach to Achieving Representative Bureaucracy
}

Adrian Cudal

San Jose State University

Follow this and additional works at: https://scholarworks.sjsu.edu/etd_projects

Part of the Policy Design, Analysis, and Evaluation Commons, Policy History, Theory, and Methods Commons, and the Public Administration Commons

\section{Recommended Citation}

Cudal, Adrian, "Diversity Management Programs: California Local Governments' Approach to Achieving Representative Bureaucracy" (2021). Master's Projects. 1036.

DOI: https://doi.org/10.31979/etd.pd4v-sq9t

https://scholarworks.sjsu.edu/etd_projects/1036

This Master's Project is brought to you for free and open access by the Master's Theses and Graduate Research at SJSU ScholarWorks. It has been accepted for inclusion in Master's Projects by an authorized administrator of SJSU ScholarWorks. For more information, please contact scholarworks@sjsu.edu. 


\section{Diversity Management Programs:}

California Local Governments' Approach to Achieving Representative Bureaucracy

by

Adrian Cudal

A Thesis Quality Research Paper

Submitted in Partial Fulfillment

of the

Requirements for the

Master's Degree

in

PUBLIC ADMINISTRATION

Professor Frances Edwards. Ph.D.

Adviser

The Graduate School

San Jose State University

December, 2021 


\section{Table of Contents}

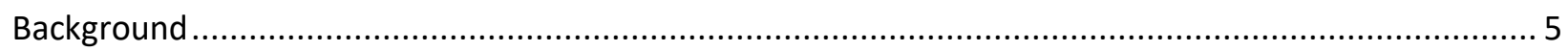

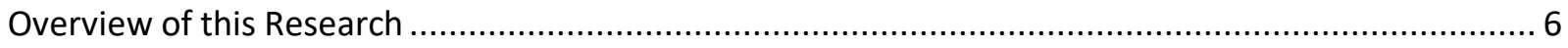

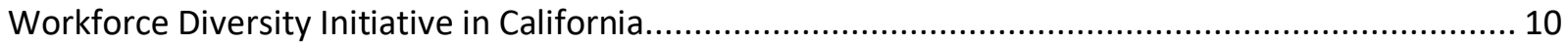

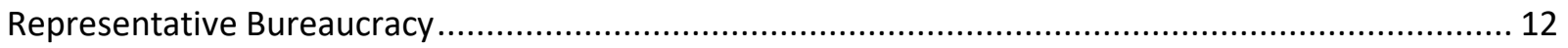

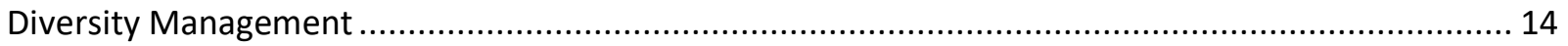

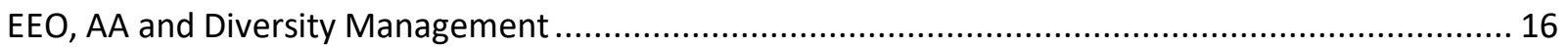

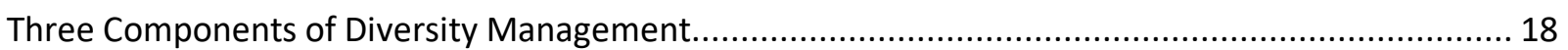

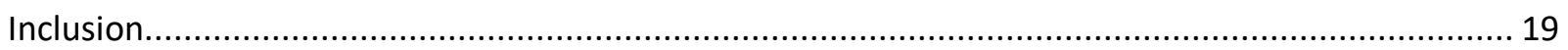

Current State of Diversity Management Initiatives................................................................ 20

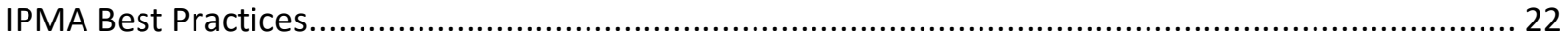

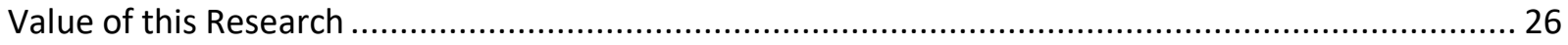

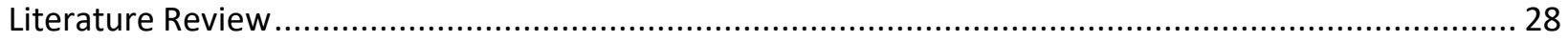

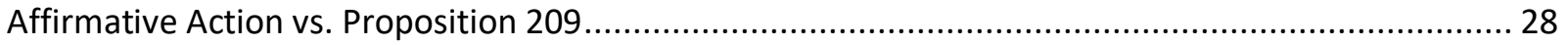

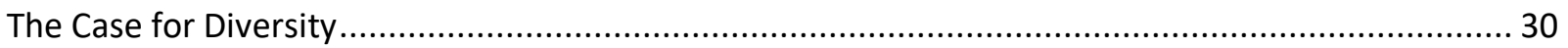

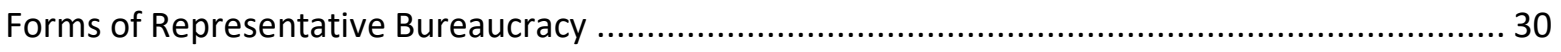

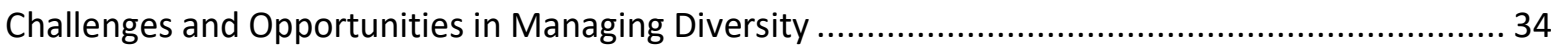

The Lack of Diversity in Bureaucratic Leadership Roles ............................................................ 38

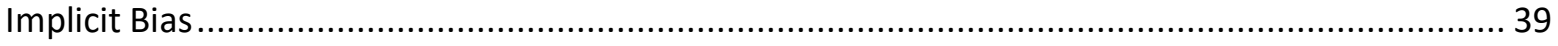

Hiring for Merit vs. Promoting Equity in the Workforce …................................................... 40

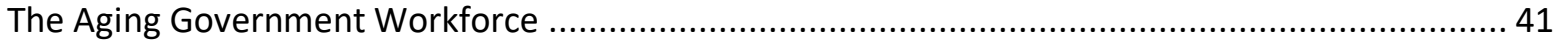

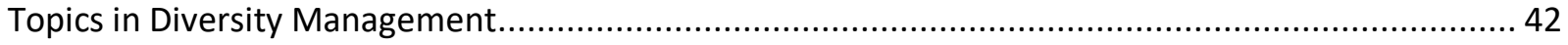

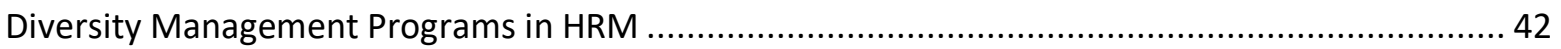

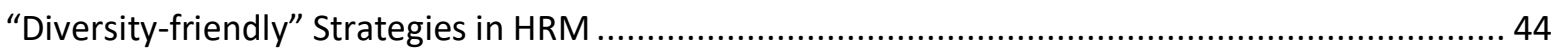

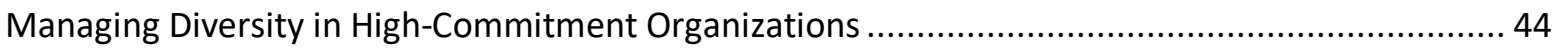

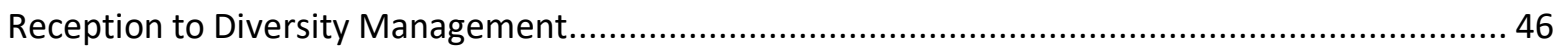

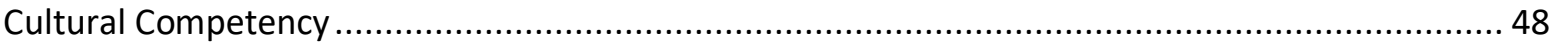

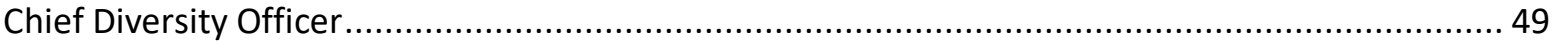

Restorative Justice Framework and Black Lives Matter ......................................................... 50

Intersectionality in Affinity Groups and Privilege Work ......................................................... 51

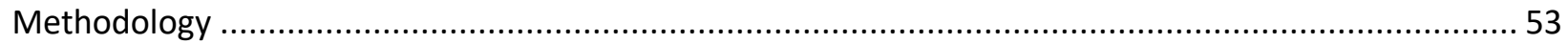

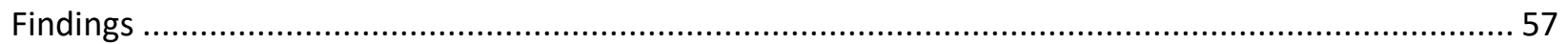

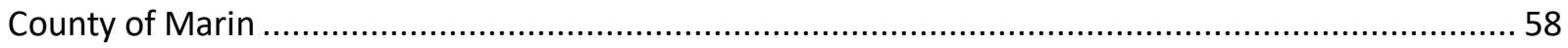




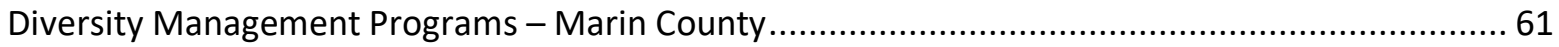

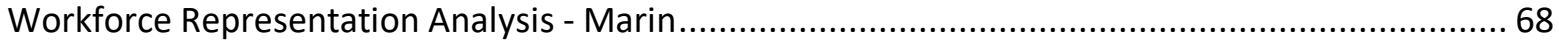

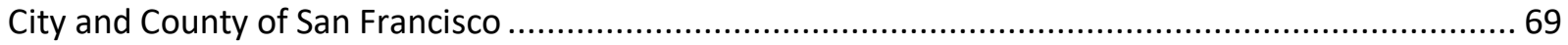

Diversity Management Programs - City and County of San Francisco........................................ 73

Workforce Representation Analysis - San Francisco ................................................................... 84

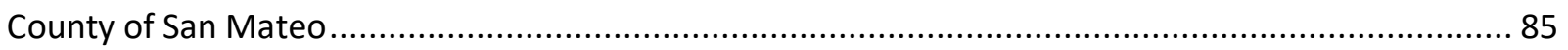

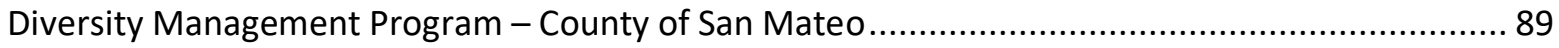

Workforce Representation Analysis - San Mateo................................................................. 95

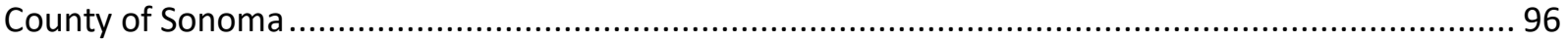

Diversity Management Programs - County of Sonoma …........................................................... 99

Workforce Representation Analysis - Sonoma …................................................................ 103

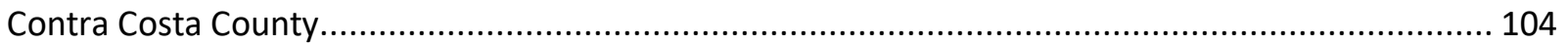

Diversity Management Programs - Contra Costa County ........................................................... 106

Workforce Representation Analysis - Contra Costa ............................................................. 111

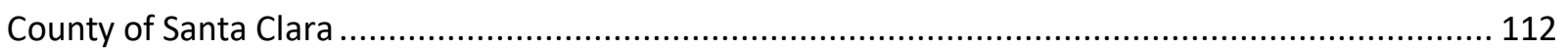

Diversity Management Programs - County of Santa Clara....................................................... 115

Workforce Representation Analysis - Santa Clara .................................................................. 120

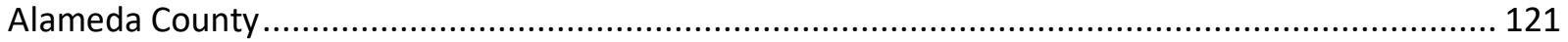

Diversity Management Programs - Alameda County ............................................................ 123

Workforce Representation Analysis - Alameda County....................................................... 125

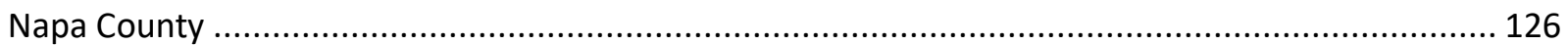

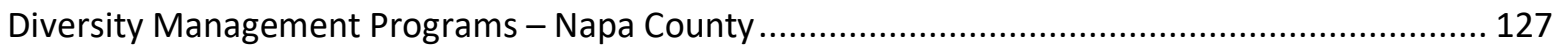

Workforce Representation Analysis - Napa County .......................................................... 128

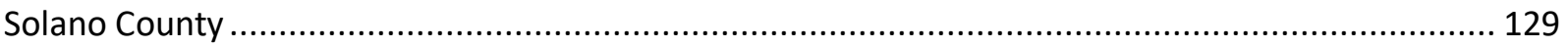

Diversity Management Program - Solano County …............................................................ 129

Workforce Representation Analysis - Solano County ........................................................... 130

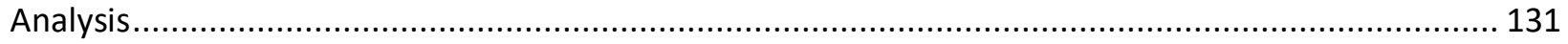

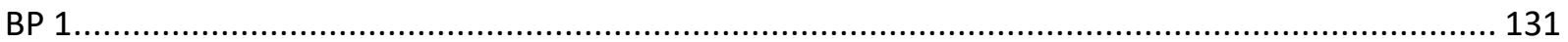

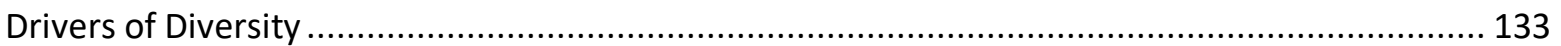

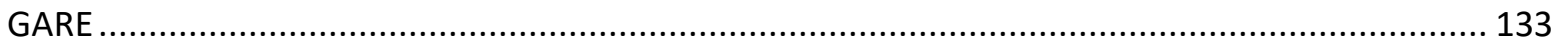

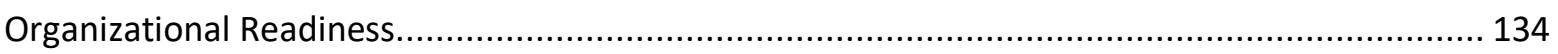

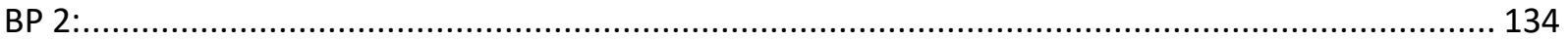

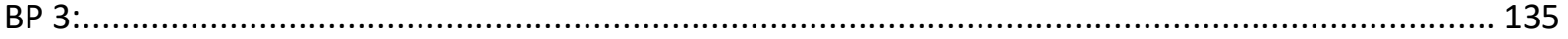




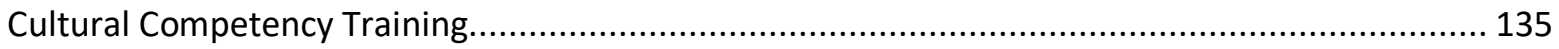

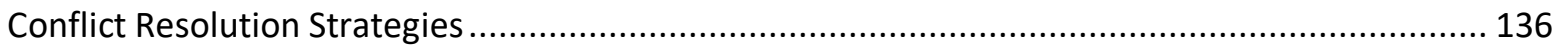

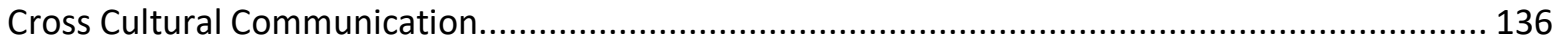

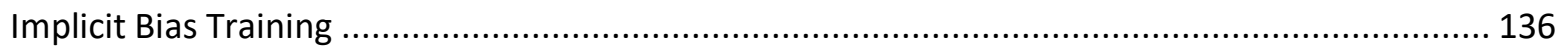

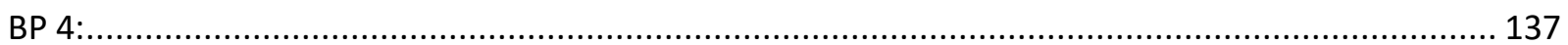

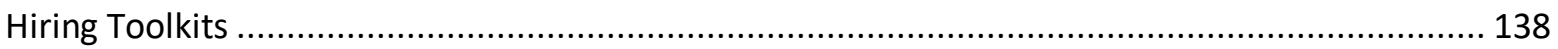

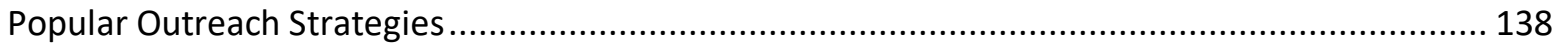

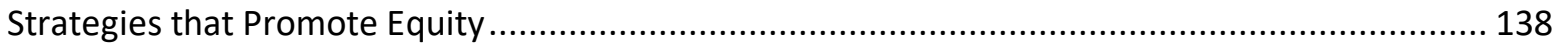

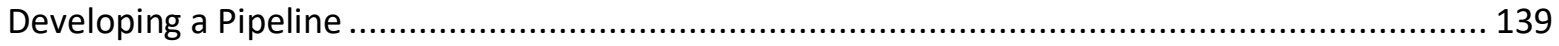

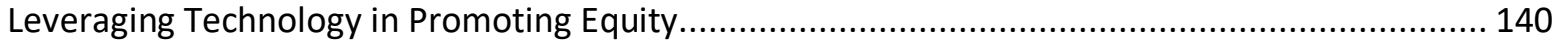

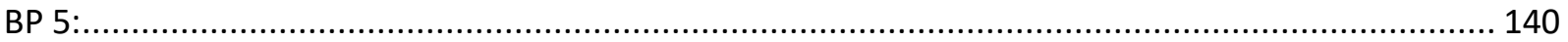

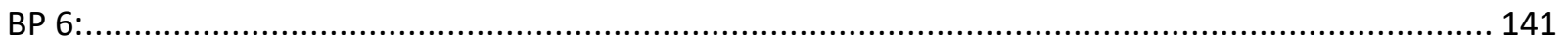

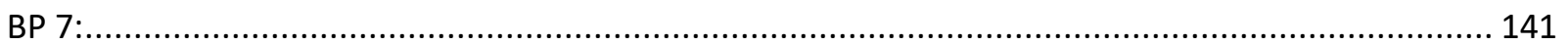

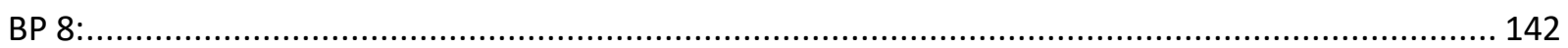

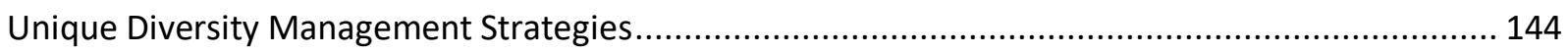

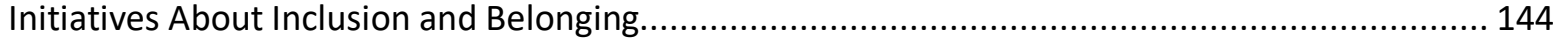

Initiatives About Equity of Opportunities and Pay ................................................................ 144

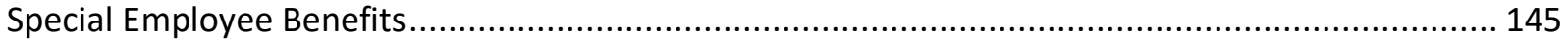

Unique Benefits (e.g., leaves, child and elder care support, flexible work locations, EAP)............ 145

Table 1: Summary of Best Practices in Bay Area Counties ....................................................... 147

Bay Area Population Breakdown ................................................................................................... 148

Table 2: Nine Bay Area Counties' Workforce By County By Race ................................................ 149

Table 3: Nine Bay Area Counties' Workforce By County By Race for Officials and Administrators..... 150

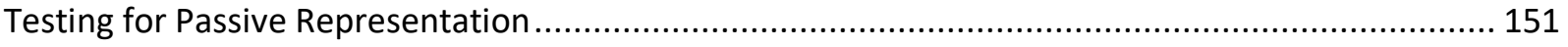

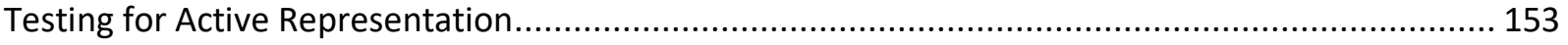

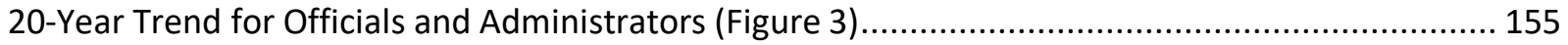

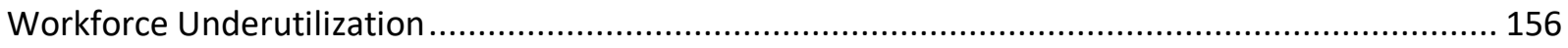

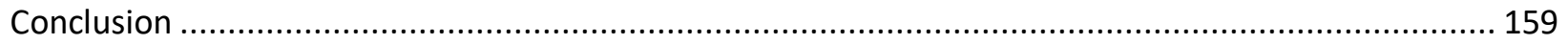

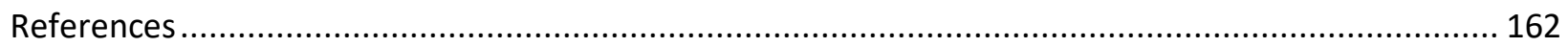




\section{BACKGROUND}

Due to its economic attractiveness and friendly immigration policies, the United States (U.S.) has continuously attracted immigrants from different parts of the globe. As a result, the U.S. is now considered one of the most ethnically diverse nations globally (Passel \& Cohn, 2008; Klingner, et al., 2010). By the year 2050, the country is predicted to further increase its racial and ethnic diversity, with the portion of non-Hispanic whites expected to decrease to roughly less than 50 percent of the entire population, while minorities and people of color, predominantly Latinos, will collectively become the new majority in the U.S. (Riccucci, 2016; Passel \& Cohn, 2008).

Researchers attribute the continually increasing population of minorities and people of color to two leading causes, immigration and differing birth rates. In 1960, immigrants in the U.S. were mostly from European countries (Klingner, et al., 2010). Nowadays most foreign-born residents are from Latin American and Asian countries, such as Mexico, the Philippines, China, Cuba, Vietnam and El Salvador (Klingner, et al., 2010, p. 167). This influx of migrants is said to be attributed to globalization, social unrest in developing countries, and the desire of immigrants to build a better life for themselves and their dependents (Klingner, et al., 2010; Rice, 2010). Immigration is said to contribute to "about half of the 2 million annual increase in American population" (Rice, 2010, p. 45). The second leading cause of the rapid population growth of minorities in the U.S. has to do with differing birth rates (Rice, 2010). Studies show that white women have a lower birth rate compared to Asian-American, African-American and Latina women (Rice, 2010). 
The rapid change in the United States' demography is expected to impact the landscape of its civilian workforce (Selden \& Selden, 2001; Riccucci, 2016). Similar to other industrialized nations, the United States' workforce is now composed of a growing number of immigrants who neither identify English as their first language nor are accustomed to traditional American culture (Klingner, et al., 2010). A Bureau of Labor Statistics projection by 2024 stated that Hispanics, Asians, and the "all other groups" category are the employee groups that are expected to have the most rapid growth, with Hispanics being the fastest growing among all race and ethnicity groups, occupying nearly one-fifth of the entire labor force (U.S. Bureau of Labor Statistics, 2015). This trend of workforce diversification is also fueled by the legal protections in employment created by laws and initiatives that espouse the virtue of social equity (Klingner, et al., 2010).

It has also been said that the public sector tends to be more diverse, and is more intentional in creating a heterogeneous organization compared to the private sector (Rice, 2010; Riccucci \& Van Ryzin, 2017; Feeney \& Camarena, 2021). Therefore, it is no surprise that underrepresented groups see career opportunities in the public sector as "a ladder of opportunity and a symbol of career mobility” (Riccucci \& Van Ryzin, 2017, p. 22).

\section{Overview of this Research}

Given these demographic trends and legislative pressures, public organizations are faced with the challenge to create a culturally competent workforce that is inclusive (Rice, 2010); who understand and value people's differences (Rice, 2010); who can promote fairness and equity, not just with the treatment of its workforce, but also with the population that it serves (Klingner, et al., 2010); and who can ensure that the organization is running efficiently and effectively as it continues to grow heterogeneously (Riccucci, 2016). Scholars in public management deemed 
that implementing diversity management programs is vital and necessary to address these challenges (Riccucci, 2016).

This research examines the existing and developing diversity management policies and programs intended to manage and promote diversity in California's nine San Francisco Bay Area counties. These diversity management programs are benchmarked to the list of diversity management best practices generated from research that was conducted by the International Personnel Management Association (IPMA) in 2001. This research seeks to answer the question, what diversity management programs and policies are currently implemented or planned in the nine Bay Area county governments, and how do they compare to the standards set by IPMA? The local government organizations that are being examined in this research include the counties of Santa Clara, San Mateo, Alameda, Contra Costa, Solano, Marin, Napa, Sonoma, and the City and County of San Francisco.

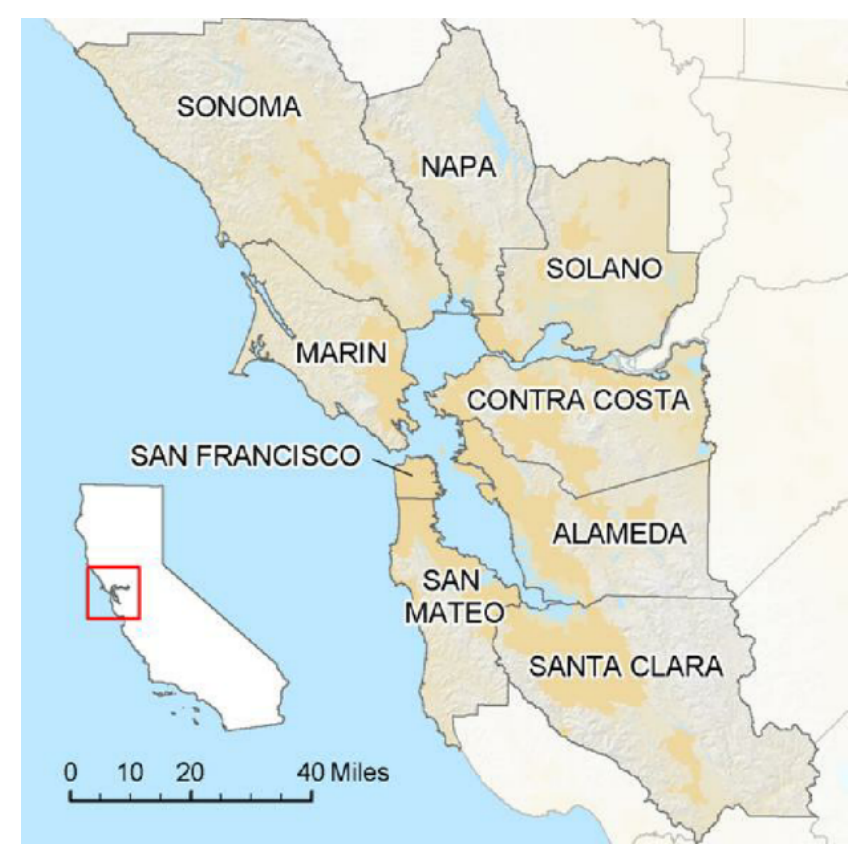

Figure 1. The Nine-County San Francisco Bay Area (2008) 
In a 2009 report titled Dominant Minority, California was identified as one of four states, including Hawaii, New Mexico and Texas, to have a "majority-minority" population (Rice, 2010). These are states where the minority population is above $50 \%$ of their entire population. In addition, the nine-county Bay Area region is considered to be the "nation's second most diverse metropolitan area out of the United States' 150 largest regions," with 59\% people of color in 2014, and 33\% predicted to be of Latino descent by the year 2050 (Bay Area Equity Atlas, 2017, p. 15).

In addition to examining the diversity management efforts of each of these county governments, this research also measures the level of representativeness of each of the nine Bay Area county governments' personnel, and examines whether the historic underrepresentation of minorities, particularly in the leadership level (Klingner, et al., 2010; Riccucci \& Van Ryzin, 2017; Feeney \& Camarena, 2021), exists within this region. It is argued that a robust diversity management program is also needed to address the prevalent lack of diversity, particularly among the "upper, higher-paying, policy making positions" which continues to be white male dominated (Riccucci, 2016, p. 51). This measurement of representativeness is done by comparing the percentage of the four major racial or ethnic employee groups (Asian, Black or African American, Hispanic or Latino and White) within the organization against the percentage of the racial or ethnic group within its jurisdiction (Riccucci \& Van Ryzin, 2017). This data provides insight to the second research question: how well are minorities represented in the Bay Area county governments compared to the constituents they serve. According to the theory of representative bureaucracy, a public organization must mirror the diversity of its constituents in order for it to effectively serve its community (Riccucci \& Van Ryzin, 2017). 
Although race is not the only consideration in the theory of representative bureaucracy, its collective capacity to influence the government makes it the most important characteristic to measure in determining the level of representativeness of a bureaucracy (Feeney \& Camarena, 2021). The four major racial groups in America that are highlighted in this research are White, African-American, Hispanic or Latino and Asian (U.S. Census, 2021).

Each of these races is defined as follows:

White - "A person having origins in any of the original peoples of Europe, the Middle East, or North Africa" (U.S. Census, 2020a).

Black or African American - "A person having origins in any of the Black racial groups of Africa” (U.S. Census, 2020a).

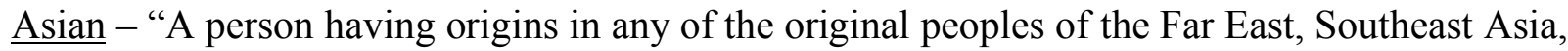
or the Indian subcontinent including, for example, Cambodia, China, India, Japan, Korea, Malaysia, Pakistan, the Philippine Islands, Thailand, and Vietnam” (U.S. Census, 2020a). Hispanics or Latino - "A person of Cuban, Mexican, Puerto Rican, South or Central American, or other Spanish culture or origin regardless of race" (U.S. Census, 2020b).

Another way to measure representation, which is commonly used particularly when developing affirmative action plans, is by comparing the employment representation of a protected group within the organization against its available labor market in the relevant community (Klingner, et al., 2010, p. 160). The third question of this research seeks to determine whether underrepresentation, or the underutilization of its available supply of labor force, is occurring within these jurisdictions. Underrepresentation (underutilization) is generally said to exist "when a particular group's employment levels within an organization are not at par with the group's presence in the relevant labor force" (Klingner, et al., 2010, p. 160). This is also a 
standard of measure used by the U.S. government in ensuring that every demographic is provided equal employment opportunity (Klingner, et al., 2010). This research measures underutilization by comparing the racial percentage of the most recent civilian workforce against the percentage of each employee racial group including its "Officials and Administrators" subgroups.

\section{Workforce Diversity Initiative in California}

Since the early 1950s, the State of California, along with the federal government, have strongly advocated for diversity and equality in the workforce through a series of laws. The state's Fair Employment Practices Act of 1959, followed by the national Civil Rights Act signed by thenPresident Lyndon Johnson in 1964, both outlawed discriminations based on race, color, religion, sex, and national origin when granting employment opportunities. The Civil Rights Act prompted the Equal Employment Opportunity Commission's creation to enforce Title VII, or the Equal Employment Opportunity (EEO) clause of this act. The provisions of this act did not apply to the public sector until its amendment in 1972 (Klingner, et al., 2010).

During the early stages of the implementation this law, it was apparent that these progressive efforts were insufficient in achieving its goal of mitigating the underrepresentation of minorities, particularly in the leadership level (Selden \& Selden, 2001). Due to this ongoing concern, compounded by the pressure caused by the threat of losing EEO oversight, the Civil Service Commission (CSC) has required the use of more aggressive affirmative action strategies such as using "goals and timetables for minority hiring and promotion" (Selden \& Selden, 2001, p. 307). This strategy of setting goals and timetables for the achievement of equal representation of minority groups in government employment was replicated across different states, including 
California, requiring organizations to consider "race, ethnicity, and gender be taken into account in employment, college admissions, and contract awards" (Selden \& Selden, 2001, p. 307).

It did not take long before equal rights advocates started contesting this practice, charging that its implementation created reverse discrimination (Klingner, et al., 2010). In 1996, 54\% of California voters approved ballot measure Proposition 209, which stated that "the state cannot discriminate against or grant preferential treatment on the basis of race, sex, color, ethnicity, or national origin in the operation of public employment, public education, and public contracting" (Ballotpedia, 2020). The passage of Proposition 209 put an end to this affirmative action practice that arguably allowed preferential treatment of minorities in California.

Even with the passage of Proposition 209 the State of California remained relentless in its goal to promote diversity in its workforce, placing emphasis on the virtue of social equity. To reaffirm this effort, Senate Bill (SB) 1045 - Public Employment and Contracting was passed by the State of California legislature, providing additional clarification on Proposition 209 provisions, particularly on how governments conduct recruitment and outreach efforts (California Legislative Information, 2002). SB 1045 allows agencies and local jurisdictions to conduct targeted outreach to attract minorities and "yield diverse results in public employment and contracting" (California Legislative Information, 2002, p. 2). Supporters of SB 1045 recognize that "California is the most diverse state in the nation, and it is important that the state not only recruit a diverse workforce, but retain it as well" (California Legislative Information, 2002, p. 5). 


\section{Representative Bureaucracy}

The desire of the public sector to attain workforce representativeness, or the equitable distribution of employment opportunities among women and minorities within the community, is founded in the theory of representative bureaucracy (Klingner, et al., 2010). This theory is premised on the idea that in order for a bureaucracy to be efficient and democratic, it is critical that the composition and origin of its workforce collectively reflect the "pluralism that exists in the society" (Rice, 2010, p. 5). A public service that is truly representative must be "inclusive of diverse races, nationalities and religions" (Rice, 2010, p. 5), and more importantly, this virtue of representativeness should also be reflected in the leadership and policy-making level (Rice, 2010).

Unlike in the private sector, where workforce diversity is solely motivated by the pressure of economic competitiveness or increasing one's bottom line, public organizations are

forced to diversify for political and practical reasons (Klingner, et al., 2010). Politically, public sector jobs are believed to be "public sector resources," as they are sustained by taxpayers' money. Therefore, they must be distributed "equitably across gender and ethnic groups so that public bureaucracies will be representative of the citizens that they serve" (Klingner, et al., 2010, p. 168). Practically, public organizations must aspire for workforce diversity so they can effectively attend to the unique service needs of their constituents (e.g. hiring Spanish-speaking staff to meet the needs of monolingual Spanish-speaking-residents) (Klingner, et al., 2010). This is why the increasing trend in workplace diversity is lauded and encouraged across different levels of the government since they all contribute to the overall goal of achieving a representative bureaucracy (The White House, 2011; California Legislative Information, 2002). 
Among the many diversity factors, such as gender, cultural differences, and age of the workforce (Rice, 2010, p. 15), scholars in public administration agree that race and ethnicity are perhaps "the most important demographic characteristic for comparing bureaucratic and public representation in the United States" (Feeney \& Camarena, 2021, p. 112). Scholars realized that different races have their own sets of values and political power that impact their decisions about certain governmental issues, such as "political alienation, childbearing, school choice, and the environment" (Rice, 2010, p. 6), and how they will receive benefits from the government (Feeney \& Camarena, 2021). Though this is what is expected, significant underrepresentation among minorities in the public workforce is still evident (Rice, 2010). According to a 1994 study, "Asian Americans are significantly underrepresented in both state and local governments" (Rice, 2010, p. 6). African Americans are also underrepresented across all levels of the government, particularly in middle and upper management (Rice, 2010).

A subset of race that is also significant when discussing representativeness is ethnicity. Asian, for instance, though treated as one category in U.S. statistics, also has different subgroups that carry with them their own values, languages, religious beliefs and even conflicts (e.g., Filipino, Chinese, Indian) (Rice, 2010). Based on a 2006 report, the United States has 300 reported ethnic groups (Rice, 2010). Among the many different facets of diversity, race and ethnicity are said to be the most challenging for many workplaces (Rice, 2010).

In today's workforce, the push for social equity is no longer just driven by "legislation, litigation and conscience;" instead, it is through the "demographic reality" and the need for a bureaucracy that is representative, that can address the needs of its multicultural population (Klingner, et al., 2010, p. 175). 


\section{Diversity Management}

The success of civil rights legislation that paved the way for inclusive labor laws, which consequently increased the "social participation, political power, and legal protection" of minorities and people of color (Klingner, et al., 2010, p. 169), along with the continuous diversification of the U.S. population due to immigration, globalization and differing birth rates (Rice, 2010), public and private organizations are faced with new sets of challenges attributed to having a workforce that will inevitably become more diverse. As a response to this trend, diversity management has been developed to effectively manage the challenges and opportunities that diversity brings (Pitts et al., 2010).

Diversity management is said to be the next phase in the series of workplace equity initiatives that go beyond the focus on hiring practices, also ensuring that "individuals of all backgrounds work harmoniously" and efficiently (Klingner, et al., 2010, p. 160). Diversity management is defined as "systematic and planned programs and procedures that are designed to improve interaction among diverse people, especially people of different ethnicities, gender and cultures" (Rice, 2010, p. 214). It achieves organizational efficiency by maximizing the potential of a diverse working environment through strategies that capitalize on diverse viewpoints (Klingner, et al., 2010). Diversity management also results in increased workforce productivity (Klingner, et al., 2010), "heightened group commitment and individual satisfaction” (Rice, 2010, p. 49). Managing diversity creates a workforce that is motivated (Klingner, et al., 2010) and more "tolerant and understanding of everyone's differences" (Rice, 2010, p. 49). It is said that diversity management would mitigate the challenges, such as "communication breakdowns, misunderstandings, and even hostilities that invariably result from working in an environment with persons from highly diverse backgrounds, age cohorts, and lifestyles" (Klingner, et al., 
2010, p. 167). Diversity management also enhances workplace performance by "breaking down the social barriers between employees and by promoting a culture of inclusion" (Riccucci \& Van Ryzin, 2017, p 23).

The definition of diversity extends beyond differences in race, ethnicity, and gender. The term also includes characteristics such as "national origin, language, religion, age, education, intelligence, and disabilities" (Klingner, et al., 2010, p. 167). Additionally, this can also include variations in socio-economic background (Klingner, et al., 2010), cultures, and sexual orientations (Rice, 2010, p. 96).

The programs, policies and initiatives that organizations implement to help promote and manage diversity are often referred to as diversity management programs. These programs are typically aimed at:

Increasing sensitivity to cultural differences; developing the ability to recognize, accept, and value diversity; minimizing patterns of inequality experienced by women and minorities; improving cross-cultural interactions and interpersonal relationships among different gender and ethnic groups; and modifying organizational culture and leadership practices. (Rice, 2010, p. 49)

The attention and urgency to address the challenges brought by diversity are believed to have started in the United States, particularly after the release of the 1987 labor market report, entitled Workforce 2000: Work and Workers for the 21st Century, which forecasted the rapidly changing demography of its population (Bassett-Jones, 2005; Ohemeng \& McGrandle, 2021). Presently, the United States' labor workforce is considered diverse and well represented; however, significant strides are still needed to be made at the leadership level, particularly for 
women and minorities (Feeney \& Camarena, 2021). In a recent 2020 U.S. Bureau of Labor Statistics report, " $84 \%$ of those in management positions in structured organizations overall identify as white, while $88 \%$ of those in chief executive positions also identify as white" (Adejumo, 2021). It is also argued that "African Americans have been at the bottom of nearly every measurable category," which is why this group is generally the focus of most diversity management initiatives (Adejumo, 2021, p. 63). However, it is also interesting to note that African Americans have a somewhat different experience in the public sector. African Americans have always given local government jobs high regard and view them as a "symbol of statute, economic power, and social mobility" (Riccucci \& Van Ryzin, 2017, p 22). African Americans, along with women, are generally well represented across different levels of the government, from federal to the local level, except for leadership (Klingner, et al., 2010). Further scrutiny on different levels of the government also reveals that, compared to women and African Americans who are both considerably well represented in the overall civilian workforce, Hispanics are the least represented among the group (Klingner, et al., 2010). Considering the importance of representativeness, this can potentially become an issue since, as stated earlier, Latinos are the fastest-growing minority group in the US.

\section{EEO, AA and Diversity Management}

As mentioned earlier, diversity management is said to be the next phase after Equal Employment Opportunity (EEO) and Affirmative Action (AA) (Klingner, et al., 2010). Equal Employment Opportunity generally refers to the policy of "treating all individuals in the employment process fair and equitably, regardless of race or gender" (Klingner, et al., 2010, p. 160). Whereas Affirmative Action refers to proactive programs meant to "improve the ability of underrepresented groups to access opportunities" and measures that grant "preferential treatment 
to individuals in target groups" (Foley \& Williamson, 2019, p. 36). Given their commonality in history and agency oversight, these two policies have caused confusion to some practitioners.

Due to its similarity to EEO and AA objectives, some see diversity management as "old wine in a new bottle" (Klingner, et al., 2010, p. 170). For compliance, some organizations are simply relabeling their AA policies and calling it diversity management (Riccucci, 2016). Some also see diversity management as an attempt to rename AA policies to make it politically palatable (Rice, 2010; Bassett-Jones, 2005) since it is centered on "American individualism" as opposed to a "state intervention" (Bassett-Jones, 2005). To better appreciate the purpose of diversity management, it would help to compare and sort out the key differences from its predecessors.

First, while EEO focuses on initiatives that prevent the violation of legal and constitutional rights of employees, and AA on the "efforts to achieve proportional representation of underrepresented groups," diversity management programs focus on the organizational objective of "increasing productivity and effectiveness" (Klingner, et al., 2010, p. 170).

Second, AA laws safeguard protected groups in the United States, such as "blacks, Hispanics, Native Americans, Asian Americans, workers over forty years old, women, and Americans with disabilities" (Klingner, et al., 2010, p. 170) to ensure that they are provided opportunities to compete for available jobs. Diversity management, on the other hand, not only focuses on the protection of these groups but, more importantly, "the economic benefit that is assumed to be inherent in a diverse workforce being 'unharmed' by the practice of segregating hierarchies within the different dimensions of workforce diversity" (Kollen, 2019, p. 259). 
Third, AA programs emphasize recruitment, selection, and promotion since these heavily influence the representation of a workforce. On the one hand, diversity management not only factors in recruitment and promotion but also "employee retention, job design, pay and benefits, education and training, and performance measurement and improvement" (Klingner, et al., 2010, p. 170).

Fourth, AA and EEO programs are created to comply with laws imposed by external agencies, which often put them in a negative light. In contrast, diversity management programs are initiatives prompted by the organizations themselves with the intent to "enhance their productivity and effectiveness" (Klingner, et al., 2010, p. 170), which can be seen as an effort that would benefit the organization's mission and culture as a whole (Klingner, et al., 2010).

In summary, EEO is meant to address workforce discrimination, AA addresses systemic racism or gender discrimination issues, and diversity management is developed to help the American workforce become more tolerant and effective in a multicultural environment (Klingner, et al., 2010).

\section{Three Components of Diversity Management}

Pitts et al. (2010) have identified three significant areas of focus of diversity management initiatives. The first is with recruitment and outreach, which concerns the recruiting efforts and strategies organizations use to seek out underrepresented applicants to increase diversity. This initiative may include conducting specialized outreach to seek talent that cannot be found in traditional outlets. The benefit of increasing diversity within an organization is based on the premise that the more diverse the staff is, the more innovative and creative the service delivery will be (Pitts et al., 2010). 
The second area of focus is the commitment to value differences. These programs are typically in diversity training and cultural awareness that promotes an appreciation of cultural differences, particularly between managers and employees. These programs emphasize that varying opinions and biases due to different cultural experiences are assets in the workforce (Pitts et al., 2010).

Lastly are pragmatic programs and policies that intend to promote workforce efficiency by enhancing employee satisfaction and productivity. These programs intend to emphasize the value of inclusivity within the organization and make "everyone" feel accepted and supported by providing "mentoring opportunities for those outside the majority organizational culture" (Pitts et al., 2010, p. 870).

\section{Inclusion}

One of the components of diversity management that has not received much attention until recently is the value of inclusion. Inclusion is considered to be the next frontier in human resource management, and has been generally identified as initiatives that focus on "emphasizing individual belongingness while at the same time encouraging individual uniqueness" (Ohemerg et al., 2021, p. 26). It also seeks to know "how employees feel about their participation and involvement in organizational processes" (Ohemeng \& McGrandle, 2021, p. 31). Inclusion is said to be the continuation of diversity management, and that it is even more effective than diversity management itself in terms of enhancing individual and organizational performance (Ohemeng \& McGrandle, 2021, p. 27). While many organizations "are better about creating diversity, many have not yet figured out how to make the environment inclusive - that is, create an atmosphere in which all people feel valued and respected and have access to the same opportunities" (Ohemeng \& McGrandle, 2021, p. 28). In spite of the success of diversity 
management in achieving representation, diverse groups still feel excluded and devalued, especially in critical conversations and decisions made within the organizations (Ohemeng \& McGrandle, 2021). Proponents say that inclusionary components are needed to ensure the success of diversity management initiatives (Ohemeng \& McGrandle, 2021). Ohemeng and McGrandle highlighted several indicators of inclusivity in the workplace, such as aempoyees' perceptions that they are vital members of a work group; that they have the capacity to influence and contribute to critical decisions and processes in the organization; and the sense of job security felt by employees which reaffirms the feeling that they are valued by the organization (Ohemeng \& McGrandle, 2021). Research in public agencies in Texas found that employees are more productive when working in environments where they can safely express their opinions and know that their opinions are valued (Ohemeng \& McGrandle, 2021).

\section{Current State of Diversity Management Initiatives}

As the workforce in both the public and private sectors becomes more diverse, industries have begun to spend significant amounts of "financial resources, time and energy into diversity missions, programs, and strategies" (Rice, 2010, p. 49). Various titles and acronyms are used to refer to these programs and oftentimes can be used interchangeably. Some call it Diversity and Inclusion (D\&I), emphasizing the value of inclusion (Boden, 2020). Others would even highlight the concept of belonging, a benefit that can be drawn from the importance of inclusion -

Diversity, Inclusion and Belonging (DIB) (Boden, 2020). Belonging is the feeling of being a part of a community. It has been said that "you can have diversity of representation without inclusion and inclusion without creating an environment in which everyone feels they actually belong" (Boden, 2020). And some also emphasize the term equity to promote fairness in the distribution 
of benefits and opportunities while ensuring that inequitable barriers do not permeate - Diversity, Equity and Inclusion (DEI) (Boden, 2020).

Though there is currently no written statute requiring all government entities to develop diversity management strategies, several public organizations have realized its importance early on and have already begun implementing diversity programs. In 2011, President Barack Obama signed Executive Order 13583 "to promote equal employment opportunity, diversity and inclusion in the federal workforce, making federal workplaces models that tap talents from all segments of the society" (U.S. EEO, 2011, p. 1). This executive order seeks to identify and adopt best practices in promoting diversity, removing barriers in employment that are inequitable (Exec Order 13583). However, some of these efforts, such as the diversity and training programs, were temporarily halted during the Trump administration, as they were deemed "divisive, unAmerican propaganda training sessions" (Adejumo, 2021).

Within the Bay Area region, several public organizations have implemented diversity management strategies. For example, Santa Clara Valley Water District (Valley Water) has created a budget specifically for a Diversity and Inclusion Master Plan in FY 2018. Valley Water believes that "critical to delivering quality products and services is a workforce that finds strength and creativity in its diversity" (Valley Water, 2021).

Diversity management is said to be one of the greatest challenges in the public sector today, and choosing to ignore this challenge could put bureaucracies at risk of losing not only their competitiveness but, most importantly, their legitimacy (Soni, 2000). 


\section{IPMA Best Practices}

As the interest in diversity management in both the public and private sectors grew, several organizations took an interest in finding out the best strategies and practices to successfully promote and sustain diversity in a workplace (Rice, 2010).

In 2001, the International Personnel Management Association (IPMA), together with the National Association of State Personnel Executives (NASPE), conducted a "human resource benchmarking project" of several public organizations nationwide (Reichenberg, 2001). This study surveyed 350 organizations from 1998 to 2000. This project aimed to identify best practices in diversity management that other organizations can replicate and use as a blueprint (Reichenberg, 2001). This survey assessed specific areas such as "recruitment and selection, recruitment and retention of staff, and training" (Rice, 2010, p 109). The project committee used the following criteria to measure the success of each program:

Successful over a period of time; produced quantitative and/or qualitative results; developed recognizable positive outcomes including customer satisfaction or some type of definitive positive impact; innovative; replicable, portable and/or transferable to other organizations with modifications. The best practice should also add value by improving service, quality, and/or productivity; and meaningful to users of the benchmarking project data. (Reichenberg, 2001, p. 1)

The states of Oklahoma, Washington, Wisconsin, and the City of Petersburg, Florida, were identified as best practice agencies (Reichenberg, 2001).

The following are the list of best practices (BP) that were identified:

BP 1: "The development of a formal process that is contained in laws, rules, or procedures. Both 
human and financial time and resources are devoted to the program. In best-practice organizations, diversity is a process that is an integrated, ongoing, and measurable strategy" (Reichenberg, 2001, p. 2). In order for diversity management efforts to be successful, "top-level commitment and organizational visibility" are needed to ascertain its "long-term impact on organization's mission or culture" (Klingner, et al., 2010, p. 172). In addition to creating laws and procedures, some of the best practice organizations have established contracts among their leadership team or have budgeted the creation of a dedicated unit or division that would push diversity initiatives and goals (Reichenberg, 2001).

BP 2: "Diversity efforts are primarily decentralized, with a central governing body outlining the requirements of the plans, with individual agencies and departments developing their own plans that are tailored to their specific needs. This reinforces a sense of ownership and ensures that managing diversity has both top-level support and is a reality throughout the organization" (Reichenberg, 2001, p. 2).

BP 3: "In best-practice organizations diversity training is provided to the workforce. The training is not limited to managers but is extended throughout the workforce. Successful organizations incorporate diversity into mentoring efforts, leadership training, and management-by-results programs" (Reichenberg, 2001, p. 2).

BP 4: "Best practice organizations utilize workforce data and demographics to compare statistics reported for the civilian labor force. Occupations with under-utilization are identified, and goals are established to reduce the under-utilization. All of the best-practice organizations use affirmative action models, but each adds creative innovations that get results and set their programs apart" (Reichenberg, 2001, p. 2). As an example, the State of Washington's Department of Personnel has a Workforce Diversity Unit that assists state agencies in 
implementing diversity enhancing strategies, such as "outreach to organizations serving groups who are underrepresented in state government; orientations that provide information about the State's recruitment procedures in general and the Workforce Diversity program in particular and special testing for individuals with language barriers" (Reichenberg, 2001, p. 5). The State of Wisconsin has hired a "full-time equal employment recruiter who helps to keep diversity in the forefront of staffing concerns" (Reichenberg, 2001, p. 6). The state also makes it a standard to announce leadership recruitment in an open competitive format to widen the possibility of getting diverse applicants (Reichenberg, 2001). Using stricter affirmative action plans, the City of St. Petersburg, Florida applies its "1-for-1" policy whenever underutilization is documented. "The policy requires that at least one member of the protected class must be hired or promoted [for] every hiring or promotion of a non-protected class member" (Reichenberg, 2001, p. 6). BP 5: "Best practice agencies have found that requiring affirmative action efforts through the law, executive order, or other mandates compels agencies to establish serious goals and to make earnest efforts toward meeting those goals" (Reichenberg, 2001, p. 3). As an example, the State Office of Personnel Management in Oklahoma is given the authority to "hold agencies accountable for implementing meaningful affirmative action efforts" (Reichenberg, 2001, p. 3). The law will compel agencies who fail to show significant progress in their diversity initiatives to take corrective actions such as "participating in special recruiting programs, developing training on equal employment, affirmative action and managing workplace diversity; or submitting all hiring and promotion decisions for mandatory review and approval" (Reichenberg, 2001, p. 3). At the time of this research, affirmative action efforts, particularly the practice of factoring race in hiring decisions in order to meet a quota, were widely accepted in several states. Presently, the State of Wisconsin is the only remaining jurisdiction among the four that has 
retained this requirement. The others have banned goals and preferential treatment in employment, like California (Ballotpedia, 2020).

BP 6: "Best practice organizations have established a review committee responsible for establishing policies, providing technical assistance, reviewing/approving plans, and monitoring progress toward achieving goals" (Reichenberg, 2001, p. 3). As an example, the State of Washington has formed a Council on Affirmative Action, composed of the Governor and legislative leader appointees responsible for advising the Secretary of the Department of Employment Relations on the progress of the affirmative action efforts. The Council meets twice a month to review the development of its programs and recommend improvements if needed (Reichenberg, 2001).

BP 7: "Effective diversity programs also link recruitment, development, and retention strategies to organizational performance. They integrate employee development processes and map career paths to see what critical skills are necessary to advance, then communicate these skills to employees and provide training" (Reichenberg, 2001, p. 3). As mentioned earlier, pragmatic efforts, such as mentoring minorities in the workplace, emphasize the organization's value of inclusivity and would make everyone feel that they are supported (Pitts et al., 2010). Research shows that having supportive leaders and training programs can contribute to the success of diversity management initiatives (Riccucci, 2016).

BP 8: "Accountability for the results of diversity programs is another attribute of best-practice organizations. Accountability is determined through metrics, surveys, focus groups, customer surveys, management, employee evaluations, and training and education evaluations. Diversity competencies may be incorporated into management systems. In this way, organizations can determine how employees deal with people of different cultures and styles, support workplace 
diversity, include diverse people in work teams, and understand the impact of diversity on business relationships. While valuing and integrating diversity are lofty goals, to be effective, organizations must use more measurable criteria to evaluate success in managing diversity" (Reichenberg, 2001, p. 3).

Additional strategies that were mentioned in Reichenberg's IPMA report include inclusivity efforts, such as the assistance that the State of Washington provides to its workforce population who are susceptible to employment discrimination, especially "racial and ethnic minorities, persons with disabilities, and persons over 40 years of age" (Reichenberg, 2001, p. 3). The state also facilitates events that demonstrate how it values cultural differences, such as "Day-On Rather than a Day-Off" in commemoration of Martin Luther King Day, which is a statewide effort that allows workers to do volunteer work during this day, instead of taking a holiday off (Reichenberg, 2001).

For the purposes of this research analysis, the term "affirmative action efforts" will only refer to initiatives and remedies that do not explicitly factor race and ethnicity in hiring decisions, since this is currently illegal in the State of California due to Proposition 209 (Ballotpedia, 2020).

\section{Value of this Research}

Despite the decades-long interest in diversity management strategies, there is relatively little research that focuses on the efforts being made by local governments. The research presented in this project can be added to this limited resource of diversity management initiatives, particularly in county government. It has also been said that research on the representativeness of employees of state and local governments is dated (Lewis \& Yoon, 2011). This research also presents more 
recent data on whether the county workforce reflects the demographics of the people that it is serving.

Interpersonal relationships can be affected by disagreements, especially when a workplace is comprised of people with varying perceptions, thinking styles, and generations (Saxena, 2014). With these demographic shifts occurring in both the public workforce and the population, this research showcases several strategies and programs that can promote a healthy coexistence between diverse individuals and take advantage of their diverse perspectives to improve the effectiveness and efficiency of an organization. California is a uniquely diverse state, and the Bay Area region can be considered as its beacon of globalization. This research can be used as a template not just for local organizations that are experiencing rapid workforce diversification, but also for nations around the world as they enter the era of globalization. It is said that "the workforce in modern industrialized nations is becoming socially more diverse" (Klingner, et al., 2010, p. 167).

This research is also a timely resource as America enters an era of reckoning, with calls for racial justice and equity moving forward. The year 2020 was a challenging year for America, politically and socially, particularly with incidents of police brutality (Schwartz, 2020), Asian hate crimes (Gover, et al., 2020), Jewish hate crimes (Nakamura, 2021), and violent incidents in the workplace, such as the Santa Clara Valley Transportation Authority massacre (Siddiqui, et al., 2021). This research carries valuable information that can help create a culturally competent workforce that values and celebrates people's differences. 


\section{LITERATURE REVIEW}

\section{Affirmative Action vs. Proposition 209}

California's intent in achieving workforce diversity is emphasized in its statutes (California Legislative Information, 2002); however, some argue that its current efforts and policies are still insufficient and sometimes inequitable in advancing diversity in the workforce, particularly in the public sector. Selden and Selden (2001) stated that the "original intent of affirmative action in the public organization was to establish a bureaucracy representative of the general population, that is, groups would be represented in government in the same proportion as their composition in the general population" (p. 308). They also argued that a "bureaucracy would be more responsive to the public interest (and will therefore better serve democratic principles) if its personnel reflect the public served in characteristics such as race, ethnicity, and gender" (p. 308). Also, having a bureaucracy that reflects its jurisdiction gives everyone in the community a sense of inclusivity and belongingness that they too play a critical part in its governing activities (Selden \& Selden, 2001). Before the approval of Proposition 209, California state agencies were required to develop affirmative action plans to address underutilization in jobs where women and minorities were underrepresented (Sumner, 2011). In addition, the State Personnel Board had what it called a "supplemental certification policy" that allowed women and minorities to be included in the employment eligible list, even if they did not rank high enough in the testing process to be in it (Sumner, 2011).

The Thelton E. Henderson Center for Social Justice (HCSJ) at the University of California, Berkeley, conducted a study covering the years 1990 to 2007, monitoring the impact of the dissolution of affirmative action on women and minorities in the State of California civil 
service. This study showed that non-Hispanic white men still stand as the majority in the California state workforce, even more so when this study was conducted. This denotes that the underrepresentation of women and minorities at the state level still exists and has worsened after Proposition 209. It was also mentioned that among minorities, Hispanics or Latinos are the ones most affected by this disparity (Sumner, 2011).

A similar study conducted by Geshekter (2008) tracking the impact of Proposition 209 on minorities, spanning from 1997 - immediately after its passage - up to 2006, showed a contrasting result. The data gathered for this research included state agencies such as the State Personnel Board and the Department of Finance. This research showed that the representation of "white employees dropped from 57\% to 50\%" (p. 315), and "blacks maintained an 11\% share of the California public labor workforce while Hispanics increased from $18 \%$ to $21 \%$ " (p. 314). Geshekter also added that this improvement could be attributed to the public sector's significant strides in improving its recruitment and screening, training, and evaluation process of its employees by making it more equitable for everyone.

The argument with affirmative action is that it is not meant to be permanent and can cease "once they have transformed employee attitude" (Myers, 2007, p. 379). However, proponents of affirmative action say that it is still needed since "labor market discrimination continues to be prevalent, despite EEO laws" and that the apparent "gaps between different groups in relative qualifications measured by educational attainment, experience, and so on likely reflect past or current discrimination" (Holzer \& Neumark, 1999, p. 535). Critics of affirmative action argue that it creates "labor market inefficiencies and/or inequities" (Holzer \& Neumark,1999, p. 535). It has also been said that affirmative action and diversity management initiatives were beneficial for white women, but not as much for minorities (Soni, 2000). 


\section{The Case for Diversity}

\section{Forms of Representative Bureaucracy}

Diversity is a "requisite for both democracy and representative bureaucracy" (Rice, 2010, p. 5). Organizations that capitalize on "diverse perspectives, knowledge, values, and abilities" can help in creating more robust policies and decision-making (Rice, 2010, p. 5). Representative bureaucracy is said to "lead to more democratic decision-making, resulting in better decisions, because it would expand the number of diversity views brought to bear on policy-making" and it will "improve bureaucratic operations and outputs by ensuring that the decisions and services are responsive to the needs of agency clientele and potential consumers, particularly members of the minority groups" (Rice, 2010, p. 6). It has also been said that decision-makers benefit from views from minorities, as it allows them to view things and situations from an unfamiliar perspective, which in the long run will provide organizational flexibility and resilience (Rice, 2010).

There are three different ways that bureaucracies can manifest their organization's representativeness. The first is through passive representation, where a bureaucratic organization strives to mirror the demographic makeup of the community that it is serving (Riccucci \& Van Ryzin, 2017). Passive representation can be measured in several ways, but the most popular method is known as representative ratio. This method compares "the percentage of women or racial and ethnic groups in a government bureaucracy with the percentage of those groups in the general population" (Riccucci \& Van Ryzin, 2017, p. 22). A perfect representation is a ratio of 1.0 wherein the proportion of an employee group matches the demographic proportion that is found in the general population. Anything higher or lower than 1.0 is considered either overrepresented or underrepresented (Riccucci \& Van Ryzin, 2017). Passive representation is 
said to satisfy the third pillar of public administration, which is social equity - along with efficiency and economy (Riccucci \& Van Ryzin, 2017). Social equity is defined as "the active commitment to fairness, justice, and equality, in the formulation of public policy, distribution of public services, and implementation of public policy" to all members of the society (ICMA, n.d.). Achieving passive representation is a testament to the effectiveness of equitable workplace policies and initiatives that intends to combat workplace discrimination (Riccucci \& Van Ryzin, 2017). Moreover, bureaucracies can serve as role models for equitable workplace practices in the community (Riccucci \& Van Ryzin, 2017).

Even though various initiatives and programs that promote passive representation have been successfully implemented across all levels of the government, its progress is argued to be rather slow, particularly with women and people of color (Riccucci \& Van Ryzin, 2017). In addition to lack of representation at leadership levels, these groups have continued to "lag in pay, and often remain segregated in traditional female or minority departments or agencies" (Riccucci \& Van Ryzin, 2017, p. 23).

Increased productivity and job satisfaction have been attributed to having a diverse or passively represented workforce, though this would only occur when "agencies practice effective diversity management strategies" (Riccucci \& Van Ryzin, 2017, p. 23) and when these efforts are backed with sufficient managerial support. Research also shows that having a diverse decision-making body in a bureaucracy could influence the equitable employment and diversification of employment for minorities (Feeney \& Camarena, 2021).

The second form of bureaucratic representativeness is through active representation. This form of representation manifests when the passively represented workforce actively advocates 
for the "needs and interest of their sociological counterparts in the general population" (Riccucci \& Van Ryzin, 2017, p. 1) using their discretionary authority to influence decisions and policy making (Riccucci \& Van Ryzin, 2017). Several studies have demonstrated the application of this theory, particularly in law enforcement. A study of a Welsh police force showed that increasing the diverse ethnic representation in the police force not only lowered the number of complaints against the police force, but also lead the officers themselves to show accountability for their own actions. This research claims that passive representation may have a "transformative effect on all police officers, thereby producing fairer, less biased, and more objective behaviors and actions in the community that they serve" (Riccucci \& Van Ryzin, 2017, p. 24). Another study in law enforcement, particularly about the reporting of sex crimes against females, showed that female victims are more cooperative in disclosing incidents to female law enforcers, who, in turn, are more aggressive in filing the case and enforcing sexual assault laws (Riccucci \& Van Ryzin, 2017). Minorities can also manifest active representation by influencing the behavior or decisions made by the majority group in decisions that concern their social counterparts, which may include decisions in employment (Riccucci \& Van Ryzin, 2017).

Active representation is said to be critical in supporting Lincoln's "government of the people, by the people, for the people" perspective, since government "could not by itself be fully effective in the community because meetings could not be assembled on the hour every day to handle continuing problems of government" (Riccucci \& Van Ryzin, 2017, p. 24). Moreover, elected or appointed officials may oftentimes fail to advocate the needs of everyone, which is why career bureaucrats are needed to step in and to use their administrative discretion to advocate for the needs, values and interests of every single member of the community (Riccucci \& Van Ryzin, 2017). According to the New Public Administration movement, "a socially 
representative bureaucracy will ensure that underserved and disenfranchised populations are not omitted from the political process of those government agencies that ultimately allocate public service in society" (Riccucci \& Van Ryzin, 2017, p. 24).

One of the concerns of representative bureaucracy is the issue of the redistribution of resources. It asks the question of whether "the interests of all groups are really served, or whether gains to one group come with an expense to others" (Riccucci \& Van Ryzin, 2017, p. 25). More specifically, will a more representative bureaucracy be "detrimental to the established majority" (Riccucci \& Van Ryzin, 2017, p. 25)? Findings show that the majority group still has the upper hand due to existing "institutionalized biases and stereotypes in the society" that favors this group (Riccucci \& Van Ryzin, 2017, p. 25). A more representative institution will help mitigate this systemic disproportion (Riccucci \& Van Ryzin, 2017).

The third and most recent form of bureaucratic representativeness is symbolic representation. Symbolic representation states that even without active representation, passive representation alone benefits society (Riccucci \& Van Ryzin, 2017). It is argued that "the social origins of a bureaucrat can produce a sense of trust and legitimacy among citizens who share those social origins, thereby resulting in cooperation from the citizens and ultimately the production of more effective policy outcomes" (Riccucci \& Van Ryzin, 2017, p. 25). Another study in law enforcement that investigates the impact of increasing the number of African American police officers showed an increase in cooperation and compliance with the law among African Americans because of enhanced trust and perception of the legitimacy of law enforcement (Riccucci \& Van Ryzin, 2017). An antithesis of this scenario could potentially lead to incidents such as police brutality that occurred in Ferguson, Missouri, in 2014 (Riccucci \& Van Ryzin, 2017). It has been said that passive representation is "indicative of shared values and 
experiences," which helps build trust (Riccucci \& Van Ryzin, 2017, p. 26). A few other benefits of symbolic representation include "psychological satisfaction with the government and its services," especially when a member from the minority group holds a very important position in the government or when a front line worker is identified as a member of that specific community (Riccucci \& Van Ryzin, 2017, p. 26).

The effect of symbolic representation can be very important in the study of public administration, particularly in policy outcomes. The success of the government's policies and initiatives can be best achieved when there are constituencies who cooperate (e.g. helping law enforcement to resolve crimes), and who are engaged in assisting the government (e.g. collaborating with the employees in service delivery) (Riccucci \& Van Ryzin, 2017). This concept of coproduction in the delivery of services can result in "cost savings for governments" and the assurance that "democracy is guaranteed to all citizens, including the disenfranchised and underprivileged" (Riccucci \& Van Ryzin, 2017).

In order to maintain and cultivate a bureaucratic workforce that actively serves in the best interest of their constituencies, effective diversity management and leadership that values representativeness are needed (Riccucci, 2016).

\section{Challenges and Opportunities in Managing Diversity}

Numerous studies have shown that having a diverse workforce comes with many advantages.

These include an "increase in the quality of group performance, creativity of ideas, cooperation, and the number of perspectives and alternatives considered" (Rice, 2010, p. 50). "Increased diversity leads to lower levels of risk aversion and better decision-making and problem-solving capability. This arises because diversity promotes a more robust critical evaluation of the first 
solution to receive substantial support" (Bassett-Jones, 2005, p. 172). Conflicts, if channeled properly, can produce diverse perspectives and alternatives with greater critical evaluation (Bassett-Jones, 2005, p. 172). Therefore, "the likelihood of adopting a sub-optimal trajectory is reduced, especially if the team's approach to systematizing creativity and problem solving is highly developed" (Bassett-Jones, 2005, p. 173). Organizations that manage their diversity well "reduce turnover, increase productivity, gain an edge in attracting talented women and minorities, increase successful marketing to underserved populations" (Rice, 2010, p. 210).

However, diversity also comes with a variety of challenges if managed poorly. In addition to the expected issues of stereotyping and prejudice against observable forms of diversity, such as race and gender (Rice, 2010), a poorly managed diverse workforce can lead to "low organizational attachment for people who perceive themselves to be different from their coworkers" (Bassett-Jones, 2005, p. 172), “decreased group integration, dissatisfaction, and turnover" (Rice, 2010, p. 50). "Miscommunication and disabling conflict" among diverse workgroups is also one of the documented issues (Rice, 2010, p. 50). Diversity can also negatively impact a group's cohesiveness; however, too much cohesion may also lead to groupthink (Bassett-Jones, 2005).

Managers are advised to consider the unique work dynamics that exist within culturally diverse or heterogeneous workgroups. Studies have shown that humans feel more comfortable interacting among peers that they perceive to have similarities to themselves (Rice, 2010). Conversely, workers tend to be more reserved and less flexible when interacting with peers that they are unfamiliar with (Rice, 2010). It has also been said that individuals who perceive themselves to be culturally dominant would often assert their dominance by controlling 
discussions and decisions, leaving minorities mum and ultimately impeding the healthy exchange of diverse perspectives (Rice, 2010).

Race is critical in the conversation of diversity management, since marginalization of racial and ethnic minorities is a significant part of the United States' history (Rice, 2010). The rapid rise of their population, intertwined with the issue of resource-sharing with the majority white European group, could lead to conflict (Rice, 2010). Moreover, one's association to a certain race or ethnic group can influence their "empowerment or marginalization, access to or exclusion from power, and the development of individual and collective perceptions, stereotypes, and identities" (Feeney \& Camarena, 2021). It has been said that "people of color face substantial structural and institutional barriers to advancement in the workforce, including lower pay, micro aggressions, barriers to powerful networks, overt racism, discrimination, stereotyping, and prejudice" (Feeney \& Camarena, 2021, p 110).

Social Identity Theory (SIT) has been very influential in the field of diversity management. This theory states that "people classify themselves and others into social categories defined by organizational membership, religious affiliation, gender, race, ethnicity, age cohort" (Feeney \& Camarena, 2021, p. 108). This tendency is attributed to pervasive structural barriers faced by women and people of color related to "subtle assumptions, attitudes, and stereotypes" (Feeney \& Camarena, 2021, p 108). This self-identification inadvertently creates in-groups and out-groups, resulting in intergroup stereotyping and rivalry (Feeney \& Camarena, 2021).

Scholars have suggested practical theories and strategies that can help manage the challenges in a multicultural environment. The first is to take advantage of the Social Identity phenomenon by using the social category of "organizational affiliation" and reframing it as a 
form of a workgroup. By making the identity of being a workgroup member more salient, individuals will be motivated to accomplish the task as part of a collective workgroup effort instead of individualized motivation driven by their social category (Rice, 2010). The second strategy has to do with one of the group diversity perspectives called Integration and Learning perspectives. This perspective emphasizes the benefits of celebrating cultural individuality and the importance of learning beliefs and behaviors to appreciate diverse perspectives that come from varying cultural heritages and experiences (Rice, 2010). The Integration and Learning perspective "represents inclusion because it satisfies employees' striving for a balance between their subjective needs for belongingness and uniqueness" and that it "moves beyond merely tolerating various groups to fostering inclusion of those groups in the organization" (Rabl, et al., 2020, p. 532). This approach to diversity "emphasizes uniqueness by valuing the expertise and contributions of individuals with different backgrounds, thereby regarding diversity as a valuable resource for learning, change and renewal" (Rabl, et al., 2020, p. 533). A research by Rabl, et al. (2020) shows that organizations that incorporate an Integration and Learning inclusion effort are seen by its employees as ethical and inspires them to perform organizational citizenship behavior and reduce their interpersonal workplace deviance (Rabl, et al., 2020). Organization citizenship is expressed by "encouraging active participation in finding better, faster, or more efficient ways of compliance beyond those legally mandated" (Rabl, et al., 2020, p. 534).

A study that analyzed the impact of workforce diversity on productivity - productivity being defined in economic terms as "the relation between input and output" (e.g. number of hours it takes to produce a good or revenue generated by an employee divided by his/her salary) (Saxena, 2014, p. 79) - suggested adopting organizational policies, such as: 
Encouraging the use of common language in the organization among the employees; conducting various motivational and mentorship programs; keeping the channels of communication open among the employees and employers; encouraging employee participation; and accepting the fact it is not the matter of culture, it is in fact a matter of quality. Therefore, for improving productivity, quality has to be maintained and not thrown out. (Saxena, 2014, p. 84)

\section{The Lack of Diversity in Bureaucratic Leadership Roles}

Even though U.S. labor laws have successfully diversified every organization's mid and lower echelons, the lack of diversity in leadership roles is still apparent. Currently, executive and policy-making positions are still dominated by white males, which is not reflective of the present demographic of the country (Riccucci, 2016).

A 2004 federal workforce data comparing a 20-year hiring record from 1984 to 2004 shows that white and male executives are still overrepresented compared to their African American and minority counterparts (Riccucci, 2009). Lack of diversity in leadership leads to poorly vetted policies and inequitable administration of programs, particularly to diverse communities, due to the lack of cultural insight (Riccucci, 2009; Feeney \& Camarena, 2021). Conversely, a leadership pool representative of the community is more apt to advocate for the needs and interests of the population that they serve (Riccucci, 2009) and could also lead to more "progressive policies aimed at diversity and inclusion through the organization" (Feeney \& Camarena, 2021, p. 106). It is also believed that having "women and people of color in key posts in government will push for the needs and interests of their counterparts in the general population" (Riccucci, 2016, p. 55), and can serve as inspiration for their counterparts who are aspiring for leadership positions (Feeney \& Camarena, 2021). Even if diversity is achieved in 
lower-level positions, the equal ability to influence a government is still undermined when the leadership is primarily composed of white men (Feeney \& Camarena, 2021). It has been argued that representative leadership is critical, especially in local government, since it is the level of government hierarchy that interacts most closely with the public (Feeney \& Camarena, 2021). A study of the representativeness of U.S. cities suggests that women and people of color are generally underrepresented in leadership levels for small and medium-size jurisdictions. However, it has been observed that minorities are more represented in larger cities that have populations between 150,000 to 250,000 (Feeney \& Camarena, 2021).

\section{Implicit Bias}

To explain why a significant disproportion in gender and race still persist in the workforce, particularly in leadership positions, experts have deferred to the study of neuroscience and social psychology and how it influences hiring practices (Segal, 2017). One of the leading causes of inequitable hiring practices, particularly towards women and minority groups, is due to implicit bias (Segal, 2017). Implicit bias, also known as unconscious bias, refers to the human tendency to make snap or unconscious decisions based on personal biases influenced by life experiences (positive or negative), culture, and messages that are received from external sources, such as media and other influences (Segal, 2017). Numerous research studies have proven that hiring decisions can implicitly be swayed by prejudicial attitudes and stereotypes against a certain race, triggered by cues such as a person's accent or a person's ethnic-sounding name (Segrest-Purkiss, et al., 2006). That is why it is also considered modern racism at the workplace since it is a less detectable type of discrimination (Segrest-Purkiss, et al., 2006). Similarly, women can also be denied career opportunities since, traditionally, they are not perceived as capable leaders, or certain roles can be too masculine for their type (Rice \& Barth, 2017). Articles in public 
administration have singled out implicit bias as "the most significant barrier for women seeking merit-based leadership positions" (Foley \& Williamson, 2019, p. 37).

The field of human resource management is fully aware of this phenomenon, and strategies have been developed to mitigate the negative effect of implicit bias in hiring practices. Techniques, such as blind application reviews, and modifying job descriptions to make them more inclusive, have been used to promote a more equitable recruitment process (Sandoval, 2018).

\section{Hiring for Merit vs. Promoting Equity in the Workforce}

Many organizations have incorporated affirmative action strategies in their recruitments to address the persistence of racial and gender inequities in employment. However, some have been reluctant, as it violates the principles of merit-based hiring (Foley \& Williamson, 2019). Meritbased hiring practice is believed to promote organizational efficiency through vetting processes that seek top talent based on skill set, which is blind to race, age, gender, or other protected characteristics (Foley \& Williamson, 2019). This standard is believed to safeguard the recruitment process from "patronage, bias and any other undue influence" (Foley \& Williamson, 2019, p. 35). However, in light of recent arguments regarding the influence of implicit bias in employment decisions, experts have begun to question the efficiency of merit-based standards.

Merit argues that the cause of underrepresentation in the labor market is not the fault of the system but rather a product of individual career choices, lack of motivation, and incompetence (Foley \& Williamson, 2019). Furthermore, merit asserts that an individual should be responsible for their successes and believes that "your problems are all your fault; and similarly, your privileges are all your achievements" (Foley \& Williamson, 2018, p. 36). 
Conversely, critics of merit-based employment practices argue that even though a person's efforts influence their success, employment advancements and opportunities within an organization are also driven by external influences, such as how leaders decide who they should mentor (Foley \& Williamson, 2019). Research has shown that men are favored to receive mentoring opportunities more than women, giving men the advantage in earning the credentials needed to become more competitive as they compete for higher roles (Foley \& Williamson, 2019). It is argued that "inequality is not the result of individual-level differences in merit" but rather is "produced and reproduced through nonconscious attitudes and stereotypes" (Foley \& Williamson, 2019).

Though merit works under the assumption that decision-makers are capable of being impartial, studies proving the influence of implicit bias in the hiring process have proven otherwise (Foley \& Williamson, 2019).

A 1999 study, entitled Are Affirmative Action Hires Less Qualified? Evidence from Employer-Employee Data on New Hires by Holzer and Neumark investigated whether affirmative action results in hiring less qualified women and people of color. The outcome of this study suggests that even though affirmative action does lead to hiring minorities who have lower educational attainment, their productivity level is still generally comparable to their white counterparts (Holzer \& Neumark,1999). This result, however, is the reverse when it came to Hispanic men, who showed weaker work performance. The authors contend that, ultimately, job performance is what is critical in assessing workforce efficiency (Holzer \& Neumark,1999).

\section{The Aging Government Workforce}

Another added factor to the diversification of the public sector workforce has to do with its aging 
workforce that is anticipated to be a part of a massive wave of retirements (Lewis \& Yoon, 2011). Although this phenomenon can be detrimental to the retention of knowledge or institutional memory, the opportunity of promoting a new breed of diverse workforce, particularly at the leadership level, can be beneficial in creating a more representative bureaucracy (Lewis \& Yoon, 2011). According to Lewis and Yoon's report, comparing state, federal and private sectors, recent trends show that "cohort replacement will make the workforce strikingly more Hispanic, but Hispanic representation in state and federal government is likely to lag behind the general economy for years to come" (Lewis \& Yoon, 2011, p 57). The Asian cohort is also expected to grow, especially in state government, but not as fast as Latinos (Lewis \& Yoon, 2011). And African Americans are expected to retain their number, slightly growing in state government (Lewis \& Yoon, 2011).

\section{Topics in Diversity Management}

\section{Diversity Management Programs in HRM}

Managing diversity is one of the biggest challenges in human resource management (HRM) (Saxena, 2014). Organizations that manage a diversifying workforce need to recognize that a change in organizational culture must occur for diversity management policies to work - this refers to the "values, assumptions, and communication patterns that characterize interaction among employees" (Klingner, et al., 2010, p. 170). This culture is molded through time and passed on through generations within the organization (Klingner, et al., 2010). To accomplish this, policies and practices need to be implemented, particularly by human resource management (Klingner, et al., 2010). 
Diversity management affects five specific areas in human resource management.

Recruitment and Retention: Similarly to some AA strategies, these policies and programs have the purpose of "increasing the applicant pool of underrepresented groups, increasing their selection rate by developing valid alternatives for tests that have disparate impact, and developing mentoring systems that encourage retention" (Klingner, et al., 2010, p. 171). Unlike AA, which focuses on meeting quotas of specific characteristics such as race and gender, diversity management factors in a broader spectrum of employee characteristics for the purposes of productivity enhancement (Klingner, et al., 2010). Recruiting is central to all diversity management program initiatives. It is considered the most controversial due to complaints of reverse discrimination, particularly from white male employees who feel threatened by public organizations' concerted efforts in hiring diverse staff (Feeney \& Camarena, 2021).

Job Design: This area includes strategies like integrating unique job features and options, such as "flexibility of work locations and schedules to attract and retain women with child and elder care responsibilities" (Klingner, et al., 2010, p. 171).

Education and Training Programs: This area includes the training of supervisors on "policies and compliance procedures, as well as identifying harassment" (Klingner, et al., 2010, p. 171), and job training for staff (Klingner, et al., 2010).

Pay and Benefit Policies: This area includes "flexible benefits, benefits for part time positions, parental leave, child and elder care support programs, and phased retirement. Employee assistance programs (EAPs) are an effective response to diversity in the workplace" (Klingner, et al., 2010, p. 171). 
Performance measurement and productivity programs: Managers and supervisors are expected to be more attuned to their employees' diverse values and motivational perspectives. Varying management styles should be considered when defining productivity (Klingner, et al., 2010).

The common goal of these HRM programs and policies is to promote organizational effectiveness by creating a culture that is inclusive, comfortable, and productive (Klingner, et al., 2010).

\section{"Diversity-friendly" Strategies in HRM}

Along with current trends in diversity management, existing organizational strategies, such as non-adversarial dispute resolution and employee involvement and participation, have proven beneficial in developing diversity in the workforce (Klingner, et al., 2010). Formal dispute resolution that incorporates "quasi-legal adversarial procedures" has been counterproductive and divisive in a diverse working environment (Klingner, et al., 2010,p 172). As an alternative, conflict resolution techniques such as "win-win negotiations and group problem solving" are not only effective but could also demonstrate the organization's commitment to "respect, tolerance, and dignity" (Klingner, et al., 2010, p 172).

Additionally, employees who "have the necessary skills, see their work as meaningful,

feel personally responsible for productivity, and have firsthand knowledge of the actual results of this labor" tend to work happily and effectively (Klingner, et al., 2010, p 172).

\section{Managing Diversity in High-Commitment Organizations}

High-commitment organizations are a type of organization where "employees are managed by outcome-driven rather than process-driven approach. This approach, in turn, is founded on 
reliance on employee skills and knowledge, rather than high levels of supervision to ensure required levels of quality and output” (Bassett-Jones, 2005, p. 169). Organizations that embrace diversity would generally opt for a high-commitment HRM due to its output orientation approach. Managing diversity directly through supervision may be too cumbersome since the "systems and processes through which they add value are too complex for managers to control" (Bassett-Jones, 2005, p. 173). Incorporating an output-oriented approach allows managers to delegate the decision-making to different individuals and teams, as they each use their skills and creativity, which is beneficial to continuous innovation and organizational improvement (Bassett-Jones, 2005). Bassett-Jones (2005) suggests that the more diverse an organization is, the more pronounced the collectivist orientation should be.

High-commitment organizations rely on creativity and innovation to stay competitive and promoting diversity in their workforce is the best way to leverage this. Japanese organizations recognize the power of diversity in innovation and organizational competitiveness and have developed diversity management strategies that would create a collectivist environment (BassettJones, 2005). These strategies encourage its workforce to think more broadly by enhancing interlevel involvement and interaction (Bassett-Jones, 2005). They also manage to maintain their industry leadership by forging "strategic alliances to infuse new ideas and know-how into their culturally homogenous corporations" (Bassett-Jones, 2005, p. 171).

To cultivate creativity and innovation in a diverse context, high-commitment organizations are prescribed to focus on "managing work routines and the creation of appropriate teams" (Bassett-Jones, 2005, p. 172). Creating teams to address increasing workload is more ideal than assigning tasks individually (Bassett-Jones, 2005). 
When recruiting for talent, a candidate's work style and work interest pertaining to their job roles should also be considered in addition to technical skills (Bassett-Jones, 2005). Selecting someone with a similar style may appeal to the organization's trust and liking at first, but this can also lead to potential homogeneity. In a high-commitment organization where lesser supervision is employed, "there is not only a need to match functional competence and personality against the requirements of the job, there is also a need to produce a balance of work preferences, attitude to risk and inclusive orientation to social identity" (Bassett-Jones, 2005, p. 173). Using these criteria when selecting a candidate through the use of "personality and work preference measures" may also help in diversifying the selections since the criteria do not factor in age, ethnicity or gender (Bassett-Jones, 2005, p. 173).

In order to gain competitive advantage, organizations that choose the high-commitment route should demonstrate their commitment to "deploying HRM strategies that are designed to foster trust and inclusiveness through effective leadership, on the one hand, with creativity and innovation techniques that capitalize on diversity to deliver an innovation advantage on the other" (Bassett-Jones, 2005, p 164). HRM systems that were recommended to help foster a collectivist environment include "Kaplan and Norton's balance scorecard (1996) and 360-degree feedback" combined with a "reward system that empowers managers to implement employee ideas" (Bassett-Jones, 2005, p. 173).

\section{Reception to Diversity Management}

When designing diversity management programs, organizations should consider how the workforce will take these initiatives at all levels. To ensure the success of diversity management initiatives, employees at every level of the organization must understand its importance and learn how to value differences (Soni, 2000). According to Vidu (2000), organizations can improve 
employee receptivity to diversity and diversity climate in several ways; "(1) Improving perception of discrimination, exclusion, and inhospitable organizational climate on the part of women and minorities; (2) making systematic and concerted efforts to communicate diversity goals to employees; and (3) training managers and supervisors to build their diversitymanagement competence" (p. 405).

Instead of highlighting diversity as a problem, organizations must emphasize its values such as "creative energy born of learning, personal and organizational growth, and innovation" or even the opportunity to get "new perspectives to old problems" (Soni, 2000, p. 405).

To promote organizational readiness and encourage program participation, organizations must first introduce to the workforce the goals and objectives behind the diversity initiatives prior to implementing the intervention. Team-building and goal-setting exercises preceding formal interventions are also recommended to promote a deeper understanding of the problem and "encourage experimentation with solutions" (Soni, 2000, p. 405).

Diversity training programs should also take into consideration the "culture shock" experienced by the white population, particularly white males, "as they encounter a drastically changed work environment, both in its demographic composition and its value system" (Rice, 2010, p. 50). These diversity training programs should help this population adjust through this difficult transition and help them cope with the challenges that diversity brings to prevent potential conflicts that may arise due to cultural differences (Rice, 2010). When designing these programs, such as diversity management leadership workshops, the format should be sensitive to topics that may be offensive or examples that are not considered meaningful or relevant to certain participants. Otherwise, this intervention may fail (Rice, 2010). Diversity-training 
programs must promote "understanding and acceptance" of differences as opposed to "criticism and blame," which could lead to further "resistance and resentment" from white males (Soni, 2000, p. 405).

\section{Cultural Competency}

According to Riccucci (2016), one critical component that continues to be absent in diversity management is cultural competency. Cultural competency requires "respect for, and understanding of, diverse ethnic and cultural groups, their histories, traditions, beliefs, and value systems in the provision and delivery of services" (Riccucci, 2016, p. 50). In addition, cultural competency should lead to "having skills and being able to use them effectively in cross-cultural situations" (Riccucci, 2016, p. 54). Riccucci (2016) states that diversity management is the key for an organization to achieve cultural competency.

Decades worth of research has made it clear that the diversification of the United States' workforce, particularly in the public sector, is inevitable (Rice, 2010). And the need to have a culturally competent workforce is greater now than ever (Riccucci, 2016). Cultural competency is defined as "a set of harmonious behaviors, attitudes, and policies that enable the organization or agency to work effectively with various racial, ethnic, religious, and linguistic groups" (Rice, 2010, p 214-215). They are "a set of cultural behaviors and attitudes integrated into the practice methods and system, agency, or its professionals that enables them to work effectively in crosscultural situations and colleagues" (Rice, 2010, p. 215).

Rice (2010) believes that culture, in general, takes time to develop and that an organization must go through a series of six stages (from negative to positive) before it can attain cultural competency (Rice, 2010). These six stages are “(1) cultural destructiveness (2) cultural 
incapacity (3) cultural blindness (4) cultural pre competence (5) cultural competency, and (6) cultural proficiency" (Rice, 2010, p. 215). Researchers posit that for the "needs and interests of all workers" to be met," a culture change within the organization is necessary (Riccucci, 2016, p. $55)$.

Cultural competency is said to impact workforce productivity positively; therefore, managers and supervisors must be provided with the proper tools and training to engage with its multicultural workforce (Riccucci, 2016). Moreover, an organization that values the needs and interests of its diverse workforce will be more likely to consider their contributions in the upper levels of the organization, which could help in diversifying that level in the organization (Riccucci, 2016).

Attaining cultural competence is the public sector's democratic obligation, not only to its workforce but most importantly to the constituents that it is serving (Riccucci, 2016). Several discussions have arisen on the linkage between cultural competency and active representation. In order to achieve active representation, "more workforce members will need to become culturally competent before they can be expected to work efficiently and appropriately with clients and constituents" (Rice, 2010, p. 245-246). To attain this, a workforce should have a sufficient number of minorities to satisfy the condition for both passive and active representation (Rice, 2010). Riccucci (2016) stated that the EEO, AA and diversity management efforts have contributed to creating culturally competent agencies.

\section{Chief Diversity Officer}

One of the many ways that bureaucracies express active representation is by hiring their own dedicated Chief Diversity Officer or CDO (Cooper \& Gerlach, 2019). CDOs have existed in the 
private sector and higher education for over two decades, and this trend is starting to proliferate in local government (Cooper \& Gerlach, 2019). Having a CDO is not only a testament to a bureaucracy's intent to address the needs and desires of its constituents, but also an "outward sign of an organization that places a premium on managing diversity" (Cooper \& Gerlach, 2019, p. 114). A 2019 research project investigating the drivers for cities to create a CDO position found that constituency pressure, particularly on cities that are more liberal and have more diverse populations, is one of the most popular reasons for its adoption (Cooper \& Gerlach, 2019).

\section{Restorative Justice Framework and Black Lives Matter}

Still, there is overwhelming evidence that black lives have not and still do not matter in the American workplace (Opie \& Roberts, 2017). In addition to the prevalent underrepresentation in leadership and discrimination in hiring, Black workers are continuously underpaid compared to their white counterparts, or are forced to work overtime but do not get paid for it (Opie \& Roberts, 2017). Contemporary workplace racism against Black people is said to exist still. Some are pressured to conform with Eurocentric workplace norms by suppressing black cultural expression or "signaling their blackness" or are "assessed as less professional if they wear their naturally textured hair to work" (Opie \& Roberts, 2017, p. 709). Black men are also said to be more readily accepted in corporate America if they appear disarming (Opie \& Roberts, 2017). To improve the value of Black lives in the workplace, Opie \& Roberts (2017) suggest using a criminal justice system strategy known as the restorative justice framework. This strategy focuses on "repairing relationships damaged by harmful behavior," typically from criminal behavior (Opie \& Roberts, 2017, p. 708). The restorative justice approach “heeds victims' voices and allows victims and offenders to work together to heal relationships harmed when the offense 
violated values rather than relying on external authorities to determine consequences" (Opie \& Roberts, 2017, p. 708). To apply this strategy in the workplace, organizations can facilitate "perspective-taking" discussions supervised by rigorously trained researchers or facilitators. Perspective-taking is defined as "the active cognitive process of imagining the world from another's vantage point or imagining oneself in another's shoes to understand their visual viewpoint, thoughts, motivations, intentions, and/or emotions" (Opie \& Roberts, 2017, p. 711). Additional strategies that can help restore trust in the workplace include robust and thoughtful leadership that is explicit on their support for the concerns of Black people and structural HR, political and symbolic changes within organizations (Opie \& Roberts, 2017).

\section{Intersectionality in Affinity Groups and Privilege Work}

Diversity management practices have generally focused on "single identity categories (i.e., women, ethnic minorities, LGBTQs)" without acknowledging the intersectionality or the multiple identities and diverse traits that coexist within each identity (Dennissen, et al., 2020). Intersectionality recognizes that "a man is never only a man, and a woman is never only a woman; he or she also has a certain age, skin color, origin, mother tongue, sexual orientation, gender identity, and so on" (Koellen, 2021, p. 264). The effects of this intersectionality remain understudied in the field of diversity management (Koellen, 2021). In a recent 2020 study analyzing diversity networks or affinity groups, it has been found that these networks “marginalize members with multiple disadvantaged identities" (Dennissen, et al., 2020, p. 219). Affinity groups, also known as employee resource groups, are single-category voluntary support groups that cater to minority employees, providing them with a safe space that will empower them to become "visible within the organization and give them a voice" (Koellen, 2021, p. 261). Dennissen, et al., (2020) recommend single-category diversity networks to find collaborative 
opportunities among different groups to discuss concepts such as "privilege work". Privilege work entails an “ongoing reflection on one's privileged status as well as the relationship to the underprivileged. Such reflections may raise the awareness of privilege, the acceptance of being privileged, and, moreover, the process of owning up to privilege" (Dennissen, et al., 2020, p. 237). 


\section{METHODOLOGY}

This research examines the diversity management programs and policies in each of the nine Bay Area county organizations by comparing them to IPMA's diversity management best practices list. This research will incorporate Sylvia \& Sylvia’s (2012) standards-based benchmarking methodology to provide structure to this comparison. The best practices used for measurements are as follows:

BP 1: The development of a formal diversity process that is contained in laws, rules, or procedures. Both human and financial time and resources are devoted to the program. In best practice organizations, diversity is a process that is an integrated, ongoing, and measurable strategy.

BP 2: Diversity efforts are primarily decentralized, with a central governing body outlining the requirements of the plans, with individual agencies and departments developing their own plans that are tailored to their specific needs. This reinforces a sense of ownership and ensures that managing diversity has both top-level support and is a reality throughout the organization.

BP 3: In best-practice organizations, diversity training is provided to the workforce. The training is not limited to managers but is extended throughout the workforce. Successful organizations incorporate diversity into mentoring efforts, leadership training, and managementby-results programs.

BP 4: Best practice organizations utilize workforce data and demographics to compare statistics reported for the civilian labor force. Occupations with under-utilization are identified, 
and goals are established to reduce the underutilization. All of the best-practice organizations add creative and innovative recruitment strategies that get results and set their programs apart.

BP 5: Best practice agencies have found that requiring diversity programs through the law, executive order, or other mandates compels agencies to establish serious goals and to make earnest efforts toward meeting those goals.

BP 6: Best practice organizations have established a review committee responsible for establishing policies, providing technical assistance, reviewing/approving plans, and monitoring progress toward achieving goals.

BP 7: Effective diversity programs also link recruitment, development, and retention strategies to organizational performance. They integrate employee development processes and map career paths to see what critical skills are necessary to advance, then communicate these skills to employees and provide training.

BP 8: Accountability for the results of diversity programs is another attribute of bestpractice organizations. Accountability is determined through metrics, surveys, focus groups, customer surveys, management, employee evaluations, and training and education evaluations.

For the purposes of this paper, the term affirmative action (AA), referenced in the original IPMA research, was not used in this research, such as in questionnaires, or in the benchmark used in the Findings section. This was intended to avoid the association of the term affirmative action to specific strategies that factor in race and gender in hiring decisions. This practice, in accordance with Proposition 209 (1997), is illegal in the State of California (Ballotpedia, 2020). 
The data on diversity management programs of each jurisdiction was retrieved by going through publicly available sources, such as county websites, recordings of County Board of Supervisors' meetings, meeting minutes, personal communication via email sent to county personnel, and by submitting Public Records Requests (California Public Records Act). A questionnaire was included in these communications and requests. All the nine Bay Area county organizations have responded to the public information requests submitted.

To measure the level of representativeness of each jurisdiction, this research applies the principle used to gauge passive representation. This is done by getting each county's representative ratio. The representative ratio compares each of the counties' workforce data to the population's demographic composition that it is serving. As mentioned earlier in the introduction, this research will focus on the level of representativeness based on race, since this is the most important consideration in the theory of representative bureaucracy. The four major races being examined are White, Black or African-American, Asian, and Hispanic or Latino. The county workforce data within this research were either taken from online workforce dashboards or were requested from county personnel departments or via Public Records Request. The county population data were taken from the U.S. Census Bureau Quick Facts portal.

In order to measure workforce underutilization for each of the counties' relevant labor market supply - particularly for historically underrepresented racial groups - this research compares each of the counties' workforce data to its countywide labor market data retrieved from the U.S. Census Bureau. The county labor market data was taken from California's 5-Year EEO Tabulation Report by county and state from 2014-2018, which is used as a benchmark for comparing gender and racial makeup of an organization's workforce. 
This research relies on simple subtraction to analyze the representativeness on these two fronts; the percentage of county employees classified by a specific race minus the percentage of the county's population or the county's labor market also classified by that race. For example, a white employee group in Santa Clara County is $17.32 \%$ and the county's labor market or overall population for white is $35.6 \% .17 .32 \%$ (minus) $35.6 \%=-18.3 \%$. This means that the White employee group or demographic is underutilized or underrepresented by $18.3 \%$. Since representation in leadership is critical in achieving a representative bureaucracy, this research also compares the racial percentage of officials and administrators within the county's workforce with the percentage of that racial group in the community that it is serving. This data will also be compared with the availability of qualified officials and administrators who reside in the local labor market. The Officials and Administrators subgroup is defined as "occupation in which employees set broad policies, exercise overall responsibility for execution of these policies, or direct individual departments or special phases of the agency operations, or provide specialized consultation on a regional, district or area basis such as Department heads" (Contra Costa, 2018).

The race data for all employees from each county is based on acquired employee selfidentification records. Jurisdictions such as Santa Clara County, where $29.41 \%$ of its officials and administrators did not disclose their race, will not be included in this comparison.

An Institutional Research Board exclusion evaluation was conducted, which determined that human subjects were not used to fulfill the needs of this research. 


\section{FINDINGS}

This section presents the diversity management programs and policies implemented or developed within the nine Bay Area counties' government organizations. Each of these plans is categorized using the eight diversity management best practices of IPMA. Additional diversity management strategies that were not highlighted in IPMA's best practices, but were cited in the previous sections of this research, are also covered. This section answers the research question: What diversity management programs and policies are used in the nine Bay Area counties' government organizations, and how do they compare with IPMA's best practices?

This research began with the seven counties with retrievable policies and programs: County of Marin, City and County of San Francisco, San Mateo County, Sonoma County, Contra Costa County, County of Santa Clara, and Alameda County. This information is followed by the counties of Solano and Napa, which currently have no diversity management programs in place. The employees of all the county organizations being examined are unionized.

Following the discussion of the different diversity management programs is a brief examination of the level of representativeness of each of the counties' workforces. This section answers the question regarding how representative these Bay Area county government organizations are with the people they are serving, and how represented each of the four largest racial and ethnic groups are compared to their available relevant labor market. 


\section{County of Marin}

The County of Marin is located in the northwestern part of the Bay Area region, with a population size of approximately 258,826 (US Census Bureau, 2021). As of 2020, Marin County has a workforce of 2,075 employees (Marin County, 2019a).

Marin County's Board of Supervisors has adopted two converging plans that contain elements that promote diversity, inclusion, and racial equity within its workforce. The first is its 5-Year Business Plan 2015-2020 which was adopted in 2015. Its vision is to make the County of Marin more responsive to the needs of its community, with a mission to make it an "adaptive organization that encourages engagement, learning and leadership at all levels" (Marin County, 2015a, p. 12). Overseen by the county's Administrative Office, this plan is a product of a collaborative effort of six employee workgroups, together with a steering committee composed of local community leaders, which assisted the employee workgroups in evaluating the employee responses collected prior to the development of the plan (Marin County, 2015a, p. 11). The foci of the county's 5-Year plan include diversity and inclusion; innovation and change; growth and development; and communication (Marin County, 2015a, p. 12).

Drivers of Creating the Plan: It was determined that their employees wanted a "more inclusive, innovative and engaged workplace" (Marin County, 2015a, p. 3). According to Board of Supervisorsmember Katie Rice, "this plan creates the foundation for excellent services and accountability that will benefit the residents for years to come” (Marin County, 2015b, p. 1).

In 2017, Marin County adopted its second plan, referred to as the Racial Equity Action Plan. The goal of this second initiative, as its name suggests, is to help institutionalize racial 
equity in both service delivery and employment practices. This action plan was drafted in 2016 in collaboration with the Government Alliance on Race and Equity (GARE), together with a cohort of 12 county employees (Marin County, 2017a). GARE is a national network of governments that assist various public entities in developing initiatives to drive racial equity in their organizations (GARE, 2021). One of the three goals of the Racial Equity Action Plan is to further the county's mission by becoming an "equitable employer where the workforce demographics reflect the demographics of the community that it serves across the breadth and depth of County positions" (Marin County, 2017a, p. 2). In doing so, the county commits to "refining personnel policies and practices to ensure that there are no artificial barriers to employment" and to teach "hiring managers about implicit bias and all county employees about structural racism and the importance of cultural intelligence” (Marin County, 2017, p. 2) The plan also recognizes the need to educate its employees on racial equity so they can understand its importance and become effective advocates; therefore, one of the goals of this plan is to provide countywide training on racial equity and inclusion (Marin County, 2017a). The 2017 Racial Equity Action Plan is a shared oversight between the Racial Equity Team composed of 2016 and 2017 GARE cohorts and the county leadership (Marin County, 2017b).

Finally, to further advance equity within its organization, the County of Marin created its first Office of Equity established within the County Administrator's Office. This office is managed under the leadership of its very first Equity Officer, who was hired in April 2019. One of the initial goals of this office is to create a new plan that would consolidate, revitalize and expand the equity efforts found in its two former plans. The development of this new plan will consider not only the input of its own workforce but also the voices of its constituency, particularly those who belong to underrepresented groups (Muse, 2020). According to Anyania 
Muse (2020), former Equity Officer, Marin County Office of Equity has found its “north star", which is to "establish County of Marin as an anti-racist, multi-cultural workforce that leads with diversity, equity, inclusion and belonging for all” (Muse, 2020).

Drivers of Creating the Plan: In its 2016 State of the County update, equity was identified as a contributing factor in income disparities and discrepancies in life expectancy in Marin County (Marin County, 2017c). According to Assistant County Administrator Angela Nicholson, the county acknowledges that "inequity has been baked into government structure through the years," and this plan gives the county a tool to address this issue. She adds that the county needs to work with the public and the employees to ensure that it represents the diversity and values of its community (Marin County, 2017c). In a recent county publication inviting community members to take part in the formation of the county's Race Equity Planning Committee, it was mentioned that the initiative to revitalize the 2017 Racial Equity Plan was due to "urgent calls for civil rights, social justice, inclusivity, diversity and equity within Marin County” (Marin County, 2020a, p. 1).

According to Richard Crawford (2020) in his 2020 Equal Employment Opportunity Plan Report, both the 5-Year Business Plan of 2015 and Racial Equity Plan of 2017 have helped increase the racial diversity of the county's workforce. It has contributed to the $20 \%$ increase in representation of people of color; $43 \%$ increase of people of color in upper management; $84 \%$ increase of representation of African Americans in upper management; and African Americans now have sufficient representation in all levels of management (Crawford, 2020). 


\section{Diversity Management Programs - Marin County}

BP 1: The development of a formal diversity process that is contained in laws, rules, or procedures. Both human and financial time and resources are devoted to the program. In best practice organizations, diversity is a process that is an integrated, ongoing, and measurable strategy.
The County of Marin has adopted, and funded, two different plans from 2015 to the present that integrate initiatives that help promote and nurture diversity within its workforce. These plans explicitly identify goals and timelines with corresponding action steps to achieve them. In addition to assigning roles and oversight to different members of its organization, the county has also funded the creation of its first Office of Equity within its County Administrator's Office. The County of Marin has also hired its first Equity Officer (Marin County, 2020b).

\section{BP 2: Diversity efforts are} primarily decentralized, with a central governing body outlining the requirements of the plans, with individual agencies and departments developing their own plans that are tailored to their specific needs. This reinforces a sense of ownership and ensures that managing diversity has both top-level support and is a reality throughout the organization.
For the 5-Year Business Plan, the County Administrative Office and the Board of Supervisors serve as the central governing body that approves the plan. Several individuals from each core department - referred to as Subject Matter Experts (SME) - are responsible for developing and delivering strategies within their respective departments. Employees referred to as Ambassadors will serve as the spokesperson to update the line staff about the plans (Nicholson, 2019).

For the Racial Equity Action Plan of 2017, the Racial Equity Team, with the support and participation of Marin County leadership, is responsible for the oversight and implementation of the organization-wide plan. The team is responsible for developing policies and procedures needed to build a strong foundation for the racial equity initiatives (Marin County, 2017b). Although there is no clear indication of a decentralized management format in the 2017 action plan, the accountability on the outcome and the fulfillment of each action step are assigned to different departments and workgroups, such as Human Resources and the 5-Year Business Plan SME's. This format seemed to have continued even after establishing the new Office of Equity and the appointment of the Equity Officer.
BP 3: In best-practice organizations, diversity training is provided to the workforce. The training is not limited to managers but is extended throughout the workforce. Successful organizations incorporate diversity into mentoring efforts, leadership
The county continues to offer cultural competency training to all employees. During this training, "participants learn how culture informs relationships, both professional and personal, and develop methods for mediating differences that may arise" (Porteshawver, 2019). Seven hundred fifty employees completed this training in year four, and as of December 2020, 97\% of its employees have already completed the program (Muse, 2020).

The Racial Equity Action Plan released in 2017 indicated that the county would provide introductory training on racial equity and inclusion followed by a continuous conversation about the topic among staff and decision-makers - elected and management. Training will include topics on the social construction of race, the history of race in government, implicit and explicit bias, and institutional and structural racism (Marin County, 2017b). These topics will be included in existing 
training, and management-byresults programs. training such as Management Academy and Leadership Academy. Additional countywide training includes Cultural Intelligence/Competency, Structural Racism (by GARE 2016 Cohort), Racial Equity Tool, Anti-bias, how to participate in hiring panel, and process-based decision making (Marin County, 2017b). Structural Racism courses would also address Marin-specific issues and relevant national issues regarding equity (Muse, 2020).

Revamped Cultural Competency Training: Cultural Intelligence Class, also known as Cultural Competency training, is now done virtually and is consolidated to just four hours (instead of 8). Participants also can select from bite-size courses if they are interested in taking a deeper dive, such as unconscious bias and emotional intelligence (Muse, 2020).

Implicit Bias Training: The 2017 Racial Equity Action Plan also mentions establishing "clear racial equity expectations for managers" (Marin County, 2017b, p. 7). This is done by "conducting training on equitable hiring practices for all hiring managers and establishing clear expectations and accountability for racially equitable workplaces. Managers are advised to use best practices to minimize bias and incorporate equity throughout all phases of the hiring process" (Marin County, 2017 b, p. 7). Also, since community engagement is one of the emphases in the 2017 Racial Equity Action Plan, the interview panel training includes Marin staff and community members participating as a panel (Marin County, 2017b).

\section{BP 4: Best practice} organizations utilize workforce data and demographics to compare statistics reported for the civilian labor force. Occupations with underutilization are identified, and goals are established to reduce the underutilization. All of the best-practice organizations add creative and innovative recruitment strategies that get results and set their programs apart.
As one of the action items in the 5-Year Business Plan, the County of Marin has created a Diversity Hiring Toolkit - a published procedure manual of Marin's overall recruitment process, available online at Marin's Human Resources website. This detailed and easy-to-use toolkit will be used by both hiring managers and human resource analysts, not just as a recruitment guide but also as a resource for strategies to recruit diverse pools of candidates. Marin's recruitment outreach efforts can be executed independently by human resources and the hiring department (Marin County, 2019b).

Here is a list of steps and unique strategies mentioned in this toolkit.

- $\quad$ Before recruitment begins, the HR analyst must identify and inform the hiring manager of any EEO underutilization occurring in the position. This information will define the targeted recruitment outreach plan that both the HR analyst and the hiring manager should execute.

- When describing the position characteristics and ideals, the recruitment team must be mindful of the word choices and descriptions that can become a potential barrier in hiring diverse candidates. An example includes describing ideal qualities about a specific individual instead of the true attributes needed to be successful in the role.

- The recruitment team will also partner with the County of Marin's affinity groups to tap into their diverse network of members. 
- The human resource analyst and hiring manager will also review the applicants' demographic data to see if there are any unintended impacts on protected genders or minority groups. This review is done before proceeding to the next step of the recruitment process.

- When interviewing, the recruitment team must send the participating panel members a demographic questionnaire to help track the panel's diversity. This strategy aligns with the 5-Year Action plan's intent to increase gender and ethnic diversity on every selection and oral board panel (Marin County, 2015a). Interview panels must have at least one man, one woman, and one person of color (Marin Public Records Request, personal communication, June 22, 2020). The diversification of the hiring panel intends "to help eliminate any potential bias from the hiring process" (Crawford, 2020). According to Equal Employment Opportunity Director Richard Crawford (2020), this strategy has increased the number of people of color in the county workforce.

- Lastly, panel members are advised to ask uniform questions across all candidates to mitigate the effect of implicit bias.

Streamlining the "talent attraction process to ensure the best quality candidate pool" is one of the strategies highlighted under the 5-Year Business Plan (Marin County, 2015a, p. 13). Here are the three action items related to this strategy (Marin County, 2015a).

- Revising minimum qualifications for county job classifications to eliminate unnecessary barriers to employment. According to County Administrator Matthew Hymel, the county has already gone through over $50 \%$ of its job classifications to eliminate artificial barriers. Minimum requirements have been revised to ensure that the county is not putting artificial or false barriers in front of people (Hymel, 2020).

- Recruitments at the Assistant Department Head and Department Head levels must ensure diversity in the candidate pool and interview diverse applicants. At least one woman and one person of color should be interviewed in each assistant department head and department head recruitment. If this does not occur, potential causes or barriers should be documented.

- The County of Marin intends to create a world-class internship program. The county's ongoing internship program is called Career Explorer Youth Program, which "helps young people gain vocational skills and experience as they think about starting a career. It is operated by the Marin County Probation Department with assistance from the county's Department of Human Resources and Department of Health and Human Services (HHS)" (Marin County, 2018). A key objective of the program is "to provide opportunities to youths who reside in communities and/or in circumstances that traditionally present barriers to employment. This may include personal experiences with the juvenile justice system, family members with experience in either the juvenile or adult justice systems, or residence in neighborhoods often impacted by crime" (Marin County, 2018). In addition to the three participating Departments mentioned above, internship opportunities are also available in the Fire Department, the Marin County Free Library, the County Counsel's Office, the County Administrator's Office, 
Marin County Parks, and the Department of Public Works” (Marin County, 2018). “The Career Explorer program creates a diverse, skilled, and local candidate pool for employment opportunities in a manner that promotes growth and development" (Marin County, 2018).

The majority of the action items mentioned above have also been included in the Racial Equity Action Plan of 2017. In a more recent Racial Equity Plan update presented to the Board in June 2020, the committee has requested a budget that would fund an initiative that will help "develop clear pathways into county employment for residents of Marin and people of color" (Marin Public Records Request, personal communication, June 22, 2020).

Career Tracks: One of the strategies mentioned in both the 5-Year Business Plan and the Racial Equity Action Plan is the partnership with local community colleges to create career paths or pipelines not only for its current employees but also for community members who would like to explore careers within the county (Marin County, 2015). Samples of these include; Partnership with Career Explorer Youth Program and creating certificate programs that would allow students to develop a career in early childhood education, emergency response services, and human services - areas within the county that needed more diverse staffing. Firefighter Pilot Program is a similar pipeline program being developed at the Fire Department to develop future diverse firefighters.

\section{Promoting Equity in Interviews}

The members of the Ambassador Group, one of the employee subgroups in the 5-Year Business plan, who have successfully completed the interview panel training, can now be invited to serve as an additional panel member for countywide interview processes. This will address the concern raised by minority panel members who feel they were only invited to comply with having a minority panel member (Nicholson, 2019).

BP 5: Best practice agencies have found that requiring diversity programs through the law, executive order, or other mandates compels agencies to establish serious goals and to make earnest efforts toward meeting those goals.
The County of Marin's Board of Supervisors has adopted the 5-Year Business Plan and Racial Equity Action Plan in 2015 and 2017, respectively. These plans emphasize the need for employees on all levels to be involved, especially the leadership. Both plans contain a series of goals, action items, measurable metrics, and a list of committees assigned for each duty to ensure accountability in the success of these plans. However, neither of the policies state any consequence for non-compliance or failure to meet a certain quota. 
BP 6: Best practice

organizations have established a review committee responsible for establishing policies, providing technical assistance, reviewing/approving plans, and monitoring progress toward achieving goals.
The 5-Year Business Plan has created a committee which they call the Employee Steering Group who will be responsible for the implementation, progress monitoring, and evaluation of the plans. The committee supports the SMEs to make sure that the initiatives are working. Employees from different levels of the county organization must apply to become a member of this group.

Based on the most recent equity strategy, the Office of Equity has created what they call Internal Racial Equity Planning Committee, which includes both the 2016 GARE core group and the newly developed Workforce Equity Champions, also known as WE Champions. The WE Champion initiative is a collaborative effort between the Office of Equity and the Organizational Development Division of Marin's Human Resources Department. Its goal is to train and develop employees to serve as ambassadors to drive the culture change towards workforce equity and belonging. Champions will learn to become thought partners for each of their Departments and the county as a whole. They will also be trained to develop their emotional intelligence and conflict resolution skills, which will support their function as peer circle/advisers to their coworkers (Muse, 2020). As of December 2020, the county has 30 WE Champions representing 19 departments (Muse, 2020).

Since the new strategy introduces the concept of community involvement, the Internal Racial Equity Planning Committee will work together with their external partners of 15 to 20 diverse community volunteers coming from traditionally underrepresented groups (Muse, 2020).

This newly created Racial Equity Planning Committee will update the 2017 Racial Equity plan and discuss solutions on current equity challenges in the organization.
BP 7: Effective diversity programs also link recruitment, development, and retention strategies to organizational performance. They integrate employee development processes and map career paths to see what critical skills are necessary to advance, then communicate these skills to employees and provide training.
The following are career development programs currently available to Marin County employees.

Leadership Academy: A partnership with the Dominican University of California for Marin employees' professional development training (Muse, 2020).

Supervisor Bootcamp: "Employees can refresh their management and supervisor skills in specific leadership areas without making a multi-day commitment" (Nicholson, 2019). "Seven bite-size sessions with updated content and a flexible delivery system, employees can sign up for the classes they need when their schedule allows" (Nicholson, 2019). Supervisor Bootcamp also includes "training on developing the coaching culture and the importance of feedback" (Nicholson, 2019).

To ensure retention of diverse leadership, the Racial Equity Action Plan also mentions its intent to promote inclusion in the workplace, particularly towards managers of color, by providing them with a supportive environment (Marin County, 2017b). 
The 5-Year Business Plan values the importance of feedback, and one of the required strategies is to get all employees trained on the county's performance evaluation process. Employees are expected to receive and provide a yearly performance evaluation (Marin, 2015) and get trained on how to write their self-evaluations (Nicholson, 2019).

Equipped with meaningful feedback from coaching and performance evaluations, employees can choose from an array of programs that can help them further develop the skills necessary to advance. Employees can choose to participate in leadership programs to learn skills such as how to manage projects. Or sign up for "stretch assignments," where employees can ask to be reassigned and trained to perform job duties outside their everyday work scope. Employees can also request to be part of mentoring programs (Nicholson, 2019). Additionally, the 5-Year Business Plan has implemented an integrated learning and performance management solution available to all county employees (TalentQuest) (Marin, 2015).

Career Ladders: the county's published a career map to illustrate progressive career paths from a single to multiple job classifications that they can use when planning their career growth within the organization (Marin, n.d.).

BP 8: Accountability for the results of diversity programs is another attribute of best-practice organizations. Accountability is determined through metrics, surveys, focus groups, customer surveys, management, employee evaluations, and training and education evaluations.
The metrics and measurable goals assigned for each action and strategy are included in both the 5-Year Business Plan and the Race Equity Action Plan. Samples include tracking the completion and delivery of various training with specific target dates, continued monitoring of the diversity of candidate pools, and the interview panel makeup (Marin, 2015). Employee feedback is collected from focus groups (e.g., Possibility Posse), surveying the outcome after taking diversity training and employee performance evaluations (Marin County, 2020).

\section{Data Monitoring}

The Equal Employment Opportunity Plan 2020 (EEOP) has joined forces with the 5-Year Business Plan and the Race Equity Action Plan to complete the diversity management plan triage. The EEOP is a detailed and proactive blueprint of how Marin's Equal Employment Opportunity office plans to address reported underutilization and significant turnover rates, particularly of women and people of color (Marin County, 2020c). Most importantly, EEOP's biggest function in this partnership is to serve as a reliable data resource for the two plans, which can help them measure the performance of their initiatives (Marin County, 2020c). This includes: workforce diversity monitoring and seeking trends on hiring, promotions, and separations; diversity in management level; collecting and analyzing recruitment data and so as the selection process to see if any potential barriers can be modified; and using qualitative data generated from exit interviews particularly from minorities to see where things can be improved (Marin County, 2020c). To further emphasize its transparency and accountability to its goals, the county has created an interactive and publicly accessible equity dashboard that includes all the workforce data disaggregated by age, gender, race, and employment level (Marin County, 2021).

Possibility Posse: The county has begun to collect qualitative information to determine the root cause of the county's challenges in retaining women and people of color. One way is by using focus groups such as "Possibility Posse" to facilitate group sessions that will help gather employee feedback. Its first session was entitled "Attention to Retention," 
which was done in cooperation with Marin County's Affinity Group. One of the recommendations drawn from this event was the need for the county "to intensify cultural competency and cultural intelligence training to be deeper and more frequent while focusing on real-life situations in department settings alongside implicit bias training" (Muse, 2020). This need aligns with the objective of Cultural Intelligence 2.0, which is the revamped version of Marin County's current cultural intelligence training program required of all of its employees (Muse, 2020).

\section{UNIQUE DIVERSITY MANAGEMENT STRATEGIES}

Initiatives Promoting Inclusion

Safe Space Brave Space Speaker Series: Live and interactive speaking engagements that both the workforce and community can participate in. These events will help educate and normalize the sometimes uncomfortable conversations about race and create a venue to allow employees to feel that they are heard, supported, and included. Instead of taking a generic cultural competency course online, these events will invite thought leaders at the forefront of equity to provide contemporary insight, leading to a more enriching and interactive learning experience. Topics include: Building an AntiRacist Society, The Intersection Between Work and Black Lives Matter, White Fragility and Othering and Belonging. Safe Space Brave Space was launched after the George Flloyd and Briana Taylor murder.

DREAM Collaborative: DREAM stands for Diversity, Respect, Encouragement, Acceptance, Marin. Its goal is to "positively influence and transform the County of Marin's organization and workforce by promoting and celebrating cross-cultural understanding and inclusivity" (Marin County, 2019c). Marin County's Affinity Group is under DREAM, and it currently has four groups.

\section{SPECIAL EMPLOYEE BENEFITS}

Employee Assistance Program (EAP)

Employee Housing Assistance Program
The county has an Employee Assistance Program that can provide counseling needs to employees and their family members on topics such as marriage, family and relationship problems, alcohol and drug abuse, emotional, personal, and stress-related concerns, child care, elder care, financial and credit problems, federal tax consultation, pre-retirement planning and personal records management (Marin County, 2019d).

The County of Marin is piloting its Employee Housing Assistance Program, which aims to enable employees to live closer to their workplace. Approximately 55\% of Marin employees live outside the county (Marin County, 2019e). The county provides up to $\$ 7500$ of financial assistance to all full-time and part-time employees, particularly those in vulnerable housing situations. This may include rent payment, security deposit, moving expenses, and short-term storage costs. 


\section{Workforce Representation Analysis - Marin}

The following data analyzes the ethnicity of the County of Marin's 2020 workforce compared to the county's overall population and its available workforce.

\section{Representation:}

- In 2020 White (60.60\%) employees had the highest representation among the four major ethnicities, followed by Hispanic or Latino $(18.90 \%)$, then by Asian (9.90\%), and Black of African American $(6.50 \%)$.

- The employee groups that exceeded their countywide population representation are Black or African American exceeding by 3.7\%, Asian exceeding by $3.3 \%$, and Hispanic or Latino exceeding by $2.6 \%$. The white employee group is the only group whose representation is below their population demographic, which is lower by $10.50 \%$.

- For the Officials and Administrators job category, both African American and Asian employee groups are overrepresented. Hispanic and White employee groups, on the other hand, are both underrepresented.

\section{Utilization:}

- The employee groups that exceeded its representation with its available workforce are Black or African American exceeding by $4.7 \%$, Asian, exceeding by $3.60 \%$, and Hispanic or Latino exceeding by $3.56 \%$. On the one hand, white employees in the County of Marin are underrepresented by $12.65 \%$.

- In terms of representation with the county's Officials and Administrators, the white employee group (63.80\%) has the highest representation among the four major ethnicities. However, they are underrepresented as compared to the available countywide supply of officials and administrators.

- All three non-white employee groups, Black or African American, Asian and Hispanic or Latino, are above the percentage of its available supply of officials and administrators in the county.

\section{MARIN COUNTY WORKFORCE}

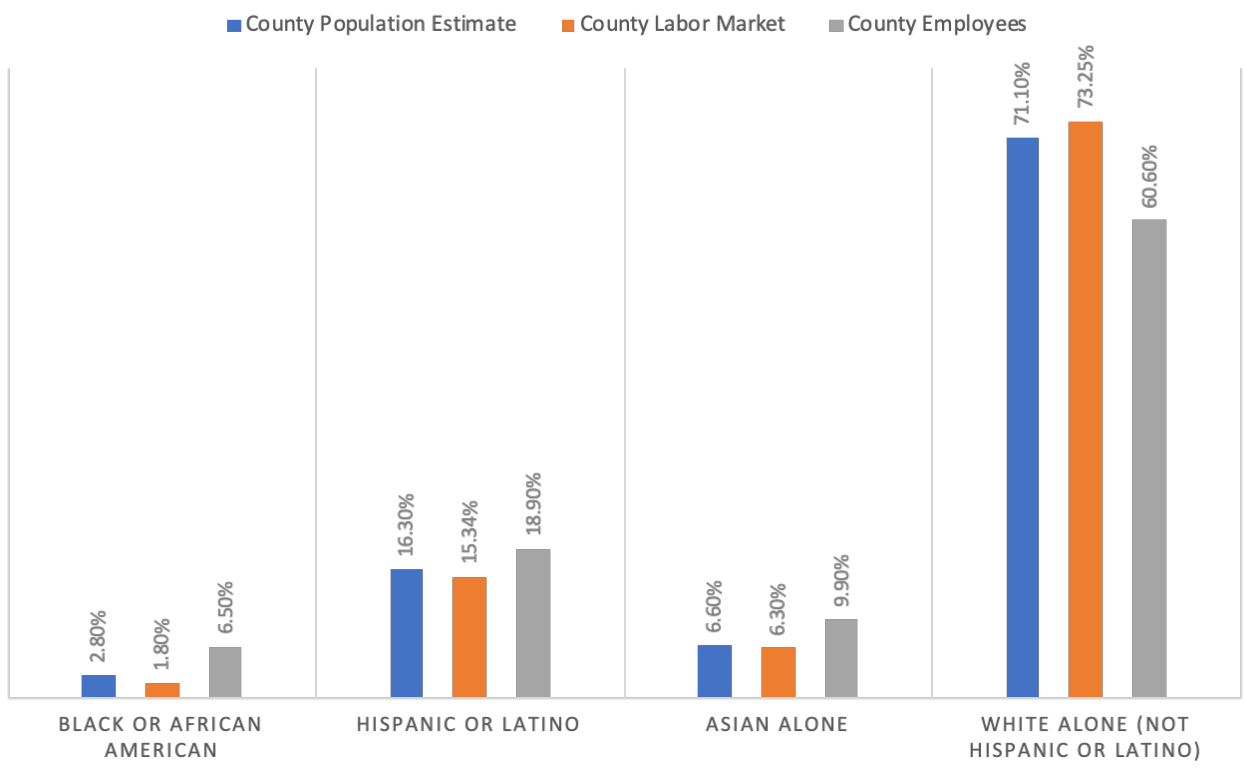

\section{OFFICIALS AND ADMINISTRATORS}

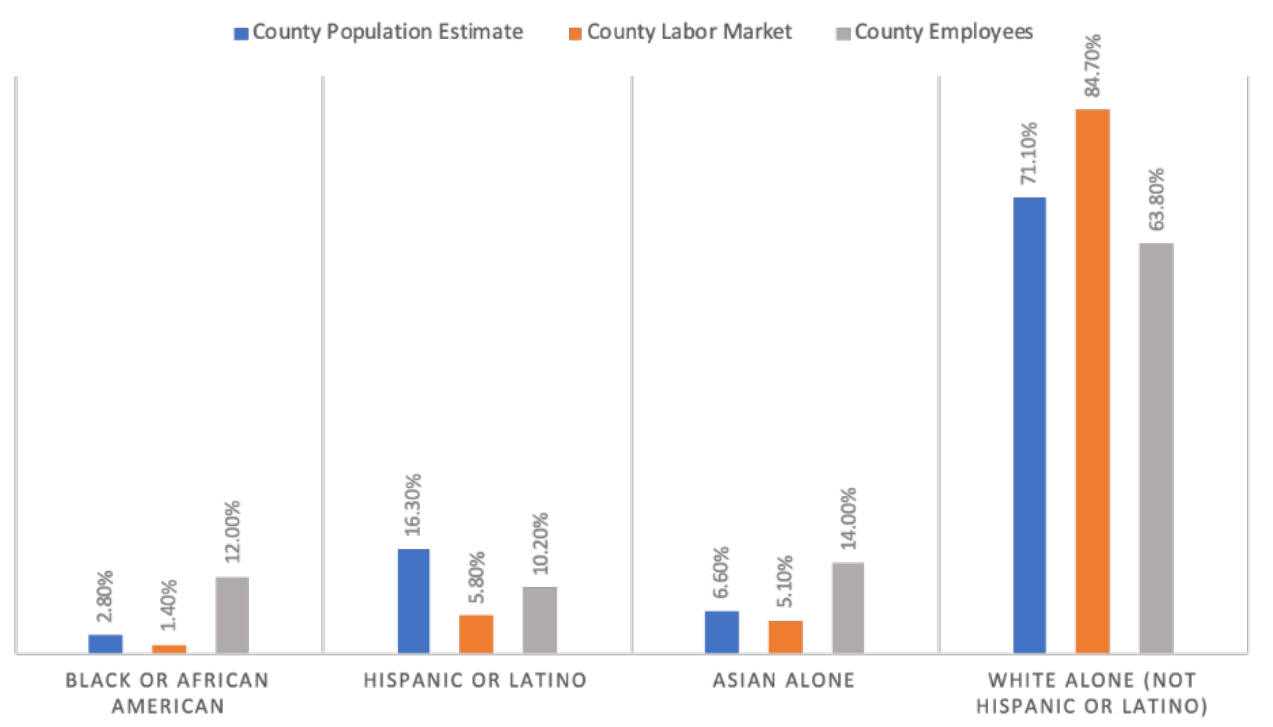




\section{City and County of San Francisco}

The City and County of San Francisco (CCSF) is considered the financial center of Northern California and is located at the north end of the San Francisco peninsula. This region has an overall population size of approximately 881,549 (US Census Bureau, 2021). As of 2020, the City and County organization has a total workforce of 35,557 , by far the largest of the nine counties in the Bay Area region (City and County, 2020).

The diversity management initiatives of the City and County of San Francisco is effectuated through the Mayor's 2018 Executive Directive 18-02, "Ensuring a Diverse, Fair and Inclusive City Workplace” (City and County, 2018), and by the 2019 Board of Supervisors Ordinance No. 188-19 - Office of Racial Equity (City and County, 2019). With the help of the Department of Human Resources, the Mayor's Executive Directive reinforces the city and county's commitment to a diverse and inclusive workplace, which ensures that all of its employees are treated with "fairness, dignity, and respect" through merit-based recruitment, free from discrimination and bias (City and County, 2018, p. 1). The Executive Directive calls to establish a cohesive and uniform citywide recruitment strategy that will actively attract a diverse pool of candidates, as opposed to the passive "post and pray" recruitment method that generally fails to attract "quality, diverse candidates the City needs to succeed in the 21 st century" (City and County, 2018, p. 1). In addition to the countywide efforts of attracting and creating a diverse workforce, the City must also be able to retain them by providing the respect and fairness that they deserve, particularly in the provision of employment opportunities and evaluations that are free from bias, harassment, and discrimination (City and County, 2018). To support this effort, the Executive Directive ordered the expansion of harassment prevention, implicit bias, and crosscultural communications training (City and County, 2018). Finally, the Mayor's Executive 
Directive values the importance of accountability and the need to have a centralized countywide data collection system that keeps track of the effectiveness of efforts to mitigate harassment, bias, and discrimination within the organization (City and County, 2018). This centralized data will consist of reports such as employee discipline and probationary releases, which will help the city, particularly the Department of Human Resources (DHR), quickly determine problem areas and act on them fast (City and County, 2018). According to the City and County of San Francisco’s DHR 2020 Annual Workforce Report, “Black and LatinX employees are disciplined at a greater rate than White or Asian employees" (City and County, 2020). In addition to data gathering, trust and accountability are also needed to make this program successful. This is demonstrated through continuous communication and collaboration of diversity, equity, and inclusion efforts among key stakeholders from different departments and parts of the organization; therefore, the executive directive also directs DHR to convene representatives from each of these sectors to discuss these efforts and how to improve them, if necessary (City and County, 2018).

Drivers of Creating the Plan: Though the city takes pride in having a workforce that surpasses the available local labor market thanks to existing programs such as anti-bias training, EEO programs, and its nationally-recognized Conviction History Program, that grants individuals equal access to job opportunities regardless of conviction history, the city admits that there is still room for improvement in certain job classifications (City and County, 2018).

In 2019, the Board of Supervisors approved Ordinance No. 188-19, which authorized the creation of the city's first Office of Racial Equity within its Human Rights Commission Department. The office is given the authority to: 
Create a citywide Racial Equity Framework, assist City departments with the development of Racial Equity Action Plans, analyze and report on the impact of the ordinance on racial equity, and carry out various other policy and reporting functions regarding racial equity; require City departments to create Racial Equity Action Plans and to provide annual updates on such Plans; require City departments to designate employees as racial equity leaders, and require the Department of Human Resources to produce an annual report concerning racial equity in the City workforce. (City and County, 2019)

In addition to its reporting function, DHR's Racial Equity Action Plan addresses its internal policies and procedures, guiding its role as the city's centralized personnel agency (City and County, 2020). DHR's Racial Equity Action Plan is a working document that aligns with the Mayor's 2018 Executive Directive, which serves as a foundation for transitioning the department from a heavily merit-system-rule driven agency to a "modern human resources business partner" (City and County, 2020, p. 2). Following the template prescribed by the Office of Racial Equity, DHR must address the following six focus areas: "hiring and recruitment; retention and promotion; discipline and separation; diverse and equitable leadership; mobility and professional development; and organizational culture of inclusion and belonging" (City and County, 2020, p. $3)$.

According to DHR's 2020 Annual Workforce Report, one of the salient challenges that the city is presently facing is how to provide equitable employment and workplace experience, particularly with its Black and Hispanic population (City and County, 2020). Given this challenge, DHR's Racial Equity Action Plan intends to concentrate on efforts that would help mitigate the inequities and improve the work-life experience of these work groups. Such 
strategies include minimizing barriers to their entry and promotion; creating a workplace climate that is more welcoming and inclusive; lessening the number of equal employment opportunity complaints, particularly from Black employees; understanding the root cause of disparities in discipline; and pay equity gaps, particularly with Black employees (City and County, 2020).

Drivers of Creating the Plan: The Office of Racial Equity (ORE) was created in response to "the City's growing racial disparities" and was a result of the combined advocacy by Black city workers, labor leaders, and community members (Office of Racial Equity, n.d., p. 1). ORE intends to address “the history of structural and institutional racism in San Francisco's delivery of services to the public and its own internal practices and systems," and to join the national movement to "address the government's role in resolving the inequitable outcomes it created" (Office of Racial Equity, n.d., p. 1). 
BP 1: The development of a formal diversity process that is contained in laws, rules, or procedures. Both human and financial time and resources are devoted to the program. In best practice organizations, diversity is a process that is an integrated, ongoing, and measurable strategy.

BP 2: Diversity efforts are primarily decentralized, with a central governing body outlining the requirements of the plans, with individual agencies and departments developing their own plans that are tailored to their specific needs. This reinforces a sense of ownership and ensures that managing diversity has both top-level support and is a reality throughout the organization.
The Mayor's 2018 Executive Directive on Ensuring a Diverse, Fair, and Inclusive City Workplace, and the Racial Equity Action Plan required by the Office of Racial Equity (ORE) from the Department of Human Resources (DHR) contain action plans, procedures and programs that both require human and financial resources. This includes creating two additional positions within DHR to form its Diversity Recruitment Team and the creation of the first Office of Racial Equity Director position. DHR's Racial Equity Action Plan has also requested a budget to help expand diversity training efforts across its workforce (City and County, 2018; City and County, 2020). ORE centers equity within the city's budget process and can make recommendations on funding of departments if certain racial equity metrics are not met (Office or Racial Equity, n.d.). Both mandates contain metrics and require DHS to provide updates and periodic reports of the progress, such as disciplinary actions, probationary releases, and performance improvement plans (City and County, 2018; City and County, 2020).
BP 3: In best-practice organizations, diversity training is provided to the workforce. The training is not limited to managers but is extended throughout the workforce. Successful organizations incorporate diversity into mentoring efforts, leadership training, and management-byresults programs.
Per Ordinance 188-19, the Office of Racial Equity will serve as the central agency to provide oversight and guidance to the citywide racial and equity efforts. Each department and agency must develop their own racial equity action plan following the framework provided by the central agency and will be responsible for their actions to improve diversity within their departments (City and County, 2019).

\section{The following is a list of required diversity training for CCSF employees.}

Michigan League for Public Policy 21-Day Racial Equity Challenge: An activity meant to promote racial equity within DHR. DHR is piloting this series that will eventually be launched on a larger scale citywide (City and County, 2020).

Fairness in Hiring: Per city policy, anyone who participates in a hiring or interview panel must take this electronic course annually. DHR has worked with the city's Controller's office to develop a dashboard to track which employee has completed the course before participating in the interview panel (City and County, 2020).

Implicit Bias Training: Per the Mayor's 2018 Executive Directive, all hiring managers and supervisors must take this course online starting January 1, 2019, and biannually after that (City and County, 2018). 
Managing Implicit Bias - Creating Awareness and Building Inclusion: In this training, "participants will understand

the effects of bias on decision-making, as they learn tools to continue creating inclusion in our daily lives" (City and

County, n.d.-a). As stated in Racial Equity Action Plan, the goal is for city department heads and commissioners to complete this course by December 31, 2019 (City and County, 2020). The names of the leadership attendees are posted on the website (City and County, n.d.-b).

\section{Optional Training}

Cross-Cultural Communications: "This one-day training helps employees explore strategies to enhance communication and build a culture of trust in their workplace. In this training, participants learn differences in communication styles and the role culture plays in communication and managing conflict" (City and County, n.d.-h).

\section{Planned Training}

CCSF is expanding the number of diversity and equity training in 2022 (San Francisco Public Records Request, personal communication, April 15, 2021).

For FY21-22: The city will "provide training and resources to deepen supervisors' and managers' learning about racism/anti-racism and other foundational DEI concepts. Establish ongoing education requirements for leaders" (City and County, 2020, p. 39).

For FY22-23: The city will "research and develop curriculum for new Equity Leadership program focused on developing leadership competencies that promote equitable, restorative, inclusive workplace environments for all employees" (City and County, 2020, p. 39).

Lastly, "The DHR Workforce Development Division is developing a learning program that will engage employees in important discussions on topics such as racism and the intergenerational trauma that racism causes" (City and County, 2020, p. 41).

BP 4: Best practice organizations utilize workforce data and demographics to compare statistics reported for the civilian labor force. Occupations with underutilization are identified, and goals are established to reduce the underutilization. All of the best-practice organizations add creative and innovative recruitment strategies that get
DHR's Diversity Recruitment Team has created a Diversity Recruitment Toolkit designed to serve as a resource to help city departments strategize their recruitment efforts towards building an inclusive and highly qualified diverse workforce. The following is information pulled from the Diversity Recruitment Toolkit provided by the city's DHR Department through the public records request received on April 16, 2021. This toolkit comes with various strategies and models that departments can use depending on their specific needs. Section one of this toolkit puts data at the forefront of the recruitment process. To measure workforce diversity, the city considers not only its relationship with the available labor pool but also migration patterns of people of color in and out of San Francisco and the effects of public policies. In doing so, this toolkit relies on a robust collection of data sources such as workforce utilization reports comparing the ethnic/racial and gender demographic of the city's workforce against the available labor pool in the eleven counties where $98 \%$ of CCSF's workforce are from. Departments also have access to DHR workforce demographic dashboards containing the demographic composition of the CCSF workforce disaggregated by department, job classification, or union information. Other sources include: 
results and set their programs

apart.
- Data SF: a public archive of city datasets that provides a comprehensive menu of city spending, geographic, funding, special projects, compensation, and other forms of data that could help inform the diversity recruitment strategies.

- 2020 Annual Workforce Report - Phase 1: The report includes demographic information on city workforce disparities across wages, corrective action, and promotional opportunities. DHR highlights measures being implemented to mitigate the effects of disproportionate trends and develop diversity, equity, and inclusion initiatives.

- JobAps Report: CCSF's application tracking system is helpful in analyzing an applicant pool's demographics and assessing how diversity decreases or increases along each stage of the hiring process. Studying where diversity decreases in the hiring process are essential to identify whether any phase of the application process may have unintended consequences in eliminating diverse candidates.

- People \& Pay Queries (eMerge): useful database for analyzing current and past employee demographics, citywide or per department.

- Metrics: to help gauge the success of the diversity efforts, different metrics are made readily available. E.g., Application Metrics, Hiring Metrics, Exit Metrics, Demographic Metrics, Internal Mobility Metrics, Learning, and Development Metrics.

Designing a Recruitment Strategy and Comprehensive Plan:

To help design a thoughtful recruitment outreach strategy, the Diversity Recruitment Toolkit asks the following questions:

- What does the current workforce look like for this particular classification and/or for the division or department as a whole? Where are there gaps in the diversity of the staff?

- What were the demographics of previous applicant pools? Which groups were missing?

- Which kinds of candidates or target audiences should we intentionally outreach to, in order to diversify our applicant pool?

- Where can we find the types of candidates we are seeking? What organizations can we work with to reach these diverse candidates?

- What are the most effective methods to communicate to the different types of candidates we are seeking? What outreach and communication strategies should we utilize?

- What is our implementation plan, including timelines and action steps for outreach and communication efforts?

- What metrics will we look at to measure the impact of our efforts? What data will we collect to assess our progress in diversifying our applicant pool along all steps of the hiring and selection process?

\section{Emphasizing Organizational Commitment to Diversity}

The toolkit also sees the importance of emphasizing the CCSF's dedication to diversity, equity, and inclusion in the community. The toolkit claims that this is a good strategy in attracting a diverse pool of candidates. Such strategies include: creating online literature on CCSF's commitment to diversity and inclusion; having senior leadership publicly express their support; organization or departmental engagement on community events attended by diverse populations, representing CCSF's diverse workforce on these community events. 


\section{Outreach Plans and Strategies}

Sourcing Diverse Candidates: DHR maintains a master source list of organizations that can be used for outreach and potential sources of diverse candidates. This includes community-based organizations and non-profit organizations; employment centers and neighborhood access centers; local universities, colleges, high schools, or vocational schools; minority-serving institutions such as Historically Black Colleges and Universities or Hispanic-Serving Institutions; and professional organizations and associations. The toolkit also recommends matching the organization to be used with the type of position that the department is recruiting for (e.g., Skilled Craft and Trades Apprenticeships - JVS, TradesWomen Inc., Neighborhood Access Center, Construction training program, Unions).

In-Person Outreach: Strategies include attending job fairs hosted by high schools, community colleges, universities, and community-based organizations that serve underrepresented populations. In-person outreach is beneficial in brand recognition and in tapping in candidate pools that have no access or prefer not to use technology. The toolkit also recommends that departments bring diverse team members to these events to help attract a diverse audience, anticipate which population will be present in the event, and bring the proper marketing materials, staffing, and culturally appropriate messaging visuals. The toolkit also recommends that the departments continuously engage with the job seekers even after the event, strategize on what information to collect, and think about follow-up mechanisms to keep diverse job seekers in the loop of future job opportunities.

Remote Outreach: The in-person restrictions imposed by the COVID-19 pandemic has opened its doors for CCSF to explore new strategies such as virtual recruiting. Benefits of remote outreach include reaching a much broader audience, cost-effectiveness, and can be integrated into one's daily tasks. Disadvantages of remote outreach include difficulty in reaching certain populations such as individuals with limited digital media literacy and individuals in fields like the skilled crafts and trades who may not have easy access to technology. In either case, outreach strategies should match the target population and be as comprehensive and inclusive as possible. Some of the available tools that DHR use include Social Media Recruiting (e.g., LinkedIn, Facebook, and Twitter), which is good in creating customized ads and targeting the right audience, especially those who are passive; promotional videos that can help applicants better understand the position, the department and their culture; and remote meeting and webinars that applicants can participate in to learn about the position and application process. After candidates are hired, webinars can also be used internally for activities such as training and onboarding. When applicants understand and have a positive experience with the city's hiring process, they are more likely to speak positively about the City and County of San Francisco as an employer of choice.

Collaboration Across Departments: DHR is interested in promoting a One City approach to recruitment, in which departments collaborate on recruitment activities, share best practices, leverage resources, and coordinate efforts. This is done in several ways: departments can share costs and tables on career fairs; participate in career fairs hosted by other departments (e.g., the Recreation and Parks job fairs, which historically attracts over 500 participants); take advantage of other agencies' networks and partnerships that can help yield diverse candidates.

Inclusive Language: In creating job announcements, recruitment materials, and when interacting with prospective candidates, the toolkit recommends the use of inclusive languages to attract a diverse audience. Such efforts include avoiding the use of gender pronouns such as "he or she" and should instead use "they" on published materials; being aware of a person's preferred gender pronoun during interactions; and the use of language software and resources such as Gender Decoder and TexTio to help in detecting gendered and less inclusive words in published literature. 
Interviews

Although not required, the HR personnel must ensure that the hiring committee is diverse and provided with the right tools and training (e.g., Fairness in Hiring training), which would equip them with the knowledge to make a sound, unbiased, and merit-based hiring decision. The city advises using a minimum of three hiring panels (e.g., culture, ethnicity, skill level, etc.) and recommends that DHR audit and make recommendations on the hiring panel if needed (City and County, 2020). The toolkit also recommends using standardized or uniform questions that are supposed to improve the quality of interviews. Hiring managers will submit their questions to HR before the interview for their approval, ensuring that they are clear and free from bias. Since CCSF is diverse, questions assessing a candidate's experience working in diverse organizations and how they value it are recommended in the toolkit. Some of the questions probe the candidate's understanding and acceptance of equity, inclusion, and diversity concepts; awareness of differences in work styles; strategies to communicate, collaborate and work effectively with diverse teams.

\section{Apprenticeships, Fellowships \& Internships}

DHR recognizes that due to lack of the required experience and skill set, not all interested applicants may qualify for its positions; therefore, career pathways were developed to help bridge this gap. Apprenticeships, fellowships, and internships allow recent graduates and people who may lack extensive employment history or employment opportunities to move from theoretical knowledge to gaining real work environment experience. These career pathway programs help develop talent, nurture commitment to public service, foster interest and early commitment to work within the City and County of San Francisco, and serve as valuable pipelines to move diverse individuals into permanent city employment. These pathway programs often attract diverse participants (City and County, 2020).

ApprenticeshipSF: "Earn and Learn" programs designed to move an apprentice from a low or no entry-level skill position to full journey level occupational proficiency. An ApprenticeshipSF program is sponsored by an individual department, city agency, or school district and is partnered with a labor organization. Upon finishing the paid training program, an apprentice earns a "Completion of Registered Apprenticeship" certificate, an industry-issued, nationally recognized credential that validates proficiency in an apprenticeable occupation. The City and County of San Francisco is the Employer of Record for over 15 registered apprenticeship programs (RAPs). Some of these jobs can be found in Automotive Technology, Landscape Maintenance, Machinery, and Parks Management (City and County, n.d.-c).

San Francisco Fellows Program: The mission of the San Francisco Fellows Program is to foster community stewardship by preparing recent college graduates and young professionals for roles in public service and administration" (City and County, n.d.-d). Fellowships are typically paid opportunities that usually lead to professional-level work.

Opportunities for All: In addition to a host of internship programs that the city provides, Opportunities for All (OFA) is specifically designed for youth and young adults affected by economic inequities in San Francisco. "The initiative includes paid internships, mentorship, and pathways to employment, including job readiness, career training, and apprenticeship for participants ages 13 to 24. OFA focuses on equitable access to these opportunities through workforce connection, support and job resources for both jobseekers and employers alike" (Opportunities for All, n.d.). 
De-identification: "DHR removes names, addresses, and schools attended from permanent civil service applications, to reduce implicit bias impacts in the interview selection process, thereby providing greater opportunity for those who may have been unfairly screened out in the past" (City and County, 2020, p. 75).

Conviction History Program: A nationally recognized program that ensures that all job applicants have a fair chance at city employment regardless of conviction history. "Conviction history information is analyzed centrally at DHR to determine whether a nexus between the specific attributes of the position and the selected candidates' conviction history exists. In addition, DHR does not disclose any non-nexus conviction history information to the department, thus mitigating opportunities for both conscious and unconscious bias" (City and County, 2020, p. 74).

With the intent to remove unintended barriers preventing diverse candidates from applying, the plan recommended DHR's Classification and Compensation team to review minimum qualifications at least once every three years (City and County, 2020).

For FY21-22: "DHR will study alternatives to the current testing formats and study whether these interventions result in a more diverse workforce while maintaining test validity and reliability" (City and County, 2020, p. 21).

"DHR currently outlines several religious observances as accepted reasons to request a make-up examination. Religious observances outlined do not require applicants to provide documentation as proof of observance" (City and County, 2020, p. 46).

"DHR human resource analysts are trained to develop job related questions with simplified language. DHR personnel ensure that only relevant questions or selection mechanisms are used" (City and County, 2020, p. 17).

BP 5: Best practice agencies have found that requiring diversity programs through the law, executive order, or other mandates compels agencies to establish serious goals and to make earnest efforts toward meeting those goals.
The Mayor's Directive on Ensuring a Diverse, Fair, and Inclusive City Workplace is an executive directive, while DHR's Racial Equity Action Plan is a product of the ORE ordinance requiring every department within the CCSF to create a racial equity action plan. Even though these directives and ordinances have resulted in creating robust action plans, neither plan states any consequence for a department's failure to follow through with its efforts to diversify.

Administrative Code (Sec. 16.9-28) requires department heads and members of city boards and commissions to complete implicit bias training.

\section{BP 6: Best practice}

organizations have established a review committee responsible for establishing policies, providing technical assistance,
Although the ORE ordinance does not establish the need to create a citywide racial equity committee, each city department is required to appoint one of their team members to become a "Racial Equity Leader" who will be responsible to coordinate the department's racial equity strategy and racial equity action plans and programs (City and County, 2019). For DHR, their racial equity action plan alluded to the Racial Equity Leader's authority to vet policies in coordination with the workgroups, e.g., Diversity Recruitment Working Group (City and County, 2020). 
BP 7: Effective diversity programs also link recruitment, development, and retention strategies to organizational performance. They integrate employee development processes and map career paths to see what critical skills are necessary to advance, then communicate these skills to employees and provide training.
As prescribed by the ORE, one of DHR's areas of focus is the execution of initiatives that promote employee mobility, professional development, retention, and promotion (City and County, 2020). Below are some of the strategies mentioned in the plan.

- HR Career Pathway: To promote equity and transparency in employee mobility, DHR-HR will develop formal pathways and standardized performance measures that departments should consider when giving employee raises and promotions. These standards will be made available to all city employees (City and County, 2020).

- DHR now recommends as best practice that "all qualified and interested employees can serve equal amounts of time in an acting role during long term absence or the period before filling a permanent vacancy. This presents an opportunity for these individuals to gain the experience of serving in an acting role and to prove their capabilities to the hiring manager. Assignments challenge employees by giving them the opportunity to work with support beyond the comfort zone of their normal work duties in order to learn new skills" (City and County, 2020, p. 28).

- DHR will "internally investigate key classifications with current drop-offs in employee diversity, such as Administrative Analyst Series (182X series). Set forth strategies and training opportunities to support employee development to achieve mobility" (City and County, 2020, p. 28).

- DHR will "revisit classifications that dead-end employees, to create a clear upward path for continued employment opportunities with the City. Publish career trajectories on the SFDHR website and include minimum qualifications for each career step. For FY 22-23: Analyze these classifications. Assess possible alternative career trajectories in other classifications" (City and County, 2020, p. 29).

- Career Development Advisor Program: “This training provides employees with an opportunity to reflect on their career goals, learn how to explore career opportunities within the City, and understand how to navigate the City's job application and exam processes. The Career Development Advisor would focus on entry level and temporary exempt employees and leading them to permanent positions within the City. Guidance would include career mapping, civil service exam preparation resources, and additional training or continuing education program references" (City and County, 2020, p. 30).

- Review Management Exam Program: review management examinations that disproportionately screen out qualified Black and LatinX candidates (City and County, 2020, p. 30).

- City University: "A partnership between the City, educational institutions, labor organizations, business, and philanthropic communities, to provide learning opportunities for City employees in an effort to produce high quality City services, improve performance, and satisfy employees. In addition, the City collaborates with these partners to provide customized training programs to address the specific needs of City departments" (City and County, n.d.-e). 
- SEIU Work Training Program: The SEIU Work Training Program is intended to "support SEIU members to pursue education that will lead to promotional opportunities. The program provides up to eight hours per week of release time for members to attend classes that (a) are only offered during the employee's regular work hours, and (b) are classes that are related to a degree, certification or other minimum qualification of a City job-class the employee aspires to" (City and County, n.d.-f).

- For FY22-23: DHR will “develop a departmental mentorship program between senior and junior level staff at DHR. Such a program would provide junior level staff with assistance, support, and feedback related to their entry or junior level positions, career development, career planning, or other growth areas" (City and County, 2020, p. 44).

- 24-Plus Training Program: This is required to be taken by new managers. This program teaches managers and supervisors how to motivate and communicate across a wide range of personalities and skill levels and be experts on city policy and procedure (City and County, n.d.-g). Supervisors and managers focus on self-management and basic models for supervision, including Situational Leadership II and coaching performance. In addition, participants learn to set expectations and provide feedback using the city's performance planning and appraisal process, addressing performance and conduct issues while managing interpersonal relations honestly and respectfully (City and County, 2020, p. 34). Additionally, DHR updated the 24-Plus training program for supervisors and managers to include content on fairness in supervision and discipline (City and County, 2020).

BP 8: Accountability for the results of diversity programs is another attribute of best-practice organizations. Accountability is determined through metrics, surveys, focus groups, customer surveys, management, employee evaluations, and training and education evaluations.
DHR will be conducting various evaluations and surveys to measure and gauge the satisfaction of its employees with the overall work environment, including diversity, equity, inclusion, and belonging (San Francisco Public Records Request, personal communication, April 15, 2021). Below are some of the strategies mentioned in the plan.

- Standardizing exit interviews to support data analysis to determine trends on why city employees leave city employment (City and County, 2020, p. 22).

- "Incorporate workforce and senior leadership demographic data into DHR's Annual report and/or other publicfacing reporting" (City and County, 2020, p. 39).

- "Implement a simple process to submit anonymous input to senior leadership. Develop a plan to respond to such input. By showing employees the feedback is acted upon, DHR hopes to encourage participation in the feedback process and assure employees there will not be subject to retribution for filing concerns" (City and County, 2020, p. 39).

- 'DHR conducted an internal staff survey focused on employees' experiences with the City's onboarding experiences, experiences that are related to retention (e.g. experiences with Professional Development opportunities; sense of belonging, engagement, and job satisfaction, etc.), and racial equity and diversity and inclusion within DHR" (City and County, 2020, p. 50).

- "The Diversity Recruitment Team works with community and nonprofit agencies who assist job seekers to identify barriers to applications. The Workforce Development Diversity Recruitment Team will also continue to seek feedback regarding outreach and recruitment efforts from over 40 partnering departments involved in its citywide Diversity Recruitment Workgroup, including OEWD who is a key partner. Ongoing meetings and workshops with employee groups may lead to more effective program implementation" (City and County, 2020, p. 54). 
- "The new Applicant Tracking System (ATS) will include job seeker and applicant feedback surveys throughout the application and hiring process. This feedback will inform updates to the system" (City and County, 2020, p. 55).

- Per Mayor Breed's Executive Order 18-02, city departments will report discipline and separations to DHR so it can analyze and early detect potential problematic areas and divisions, particularly the ones associated with biases against staff of color, so they can quickly be addressed (City and County, 2020).

- ATS will monitor the effect of recruitment outreach plans to see where applicants are engaging and track and evaluate data on race/ethnicity on the application process to address any fallout due to bias (City and County, 2020).

\section{UNIQUE DIVERSITY MANAGEMENT STRATEGIES}

\section{Initiatives Promoting Inclusion}

- The city will "support and provide spaces for affinity groups, prioritizing historically marginalized peoples" and "promote the benefits of employee affinity groups" (City and County, 2020, p. 49).

- The city will "ensure that all art, decor, and design where staff work daily reflect racial and social diversity. DHR will create a representative art committee to review the current inventory of existing art by theme and artist and identify gaps or needs. Planned for FY23-24, with additional support in the form of a workorder with the Arts Commission, change art as necessary to ensure representation of all cultures" (City and County, 2020, p. 50).

- "Review the Paid Time Off (PTO) policy annually and enhance it to value all religious and cultural holidays Additional legal holidays would need to be declared by the Mayor or Board of Supervisors. Employees currently have broad discretion to use their discretionary leave for religious and cultural holidays" (City and County, 2020, p. 27).

- "Include opportunities to expand collective knowledge regarding diversity, equity, and inclusion. DHR will provide training opportunities or speaker's series to participants in the fellows program to engage with the topic of diversity, equity, and inclusion" (City and County, 2020, p. 16).

\section{Initiatives Promoting Equit}

\section{$\underline{\text { Initiative on Retention }}$}

"Ensure salaries and benefits allow for a dignified livelihood, especially for People of Color and women. Conduct annual internal reviews of salary standards against industry standards to ensure parity. DHR continues to implement provisions of the Fair Pay Act to ensure the City provides equal pay for work of equal value regardless of gender or race. This included reiterating to departments the importance of assessing internal equity when making new appointments with current incumbents" (City and County, 2020, p. 26).

\section{Equitable Learning and Development}

"DHR's Instructional Design team collaborates with subject matter experts in DHR and other City departments to create eLearning modules that help City employees understand important citywide policies and expectations such as the City's telecommuting policies, workplace ergonomic standards, performance management processes, and COVID-19 health and 
Hiring and Recruitment Plans under the Racial Equity Action Plan

- The Diversity Recruitment Team will survey its community partners to identify the pain points faced by individuals seeking city employment and use this data to inform future policy and process changes (City and County, 2020, p. 11).

- DHR will develop questions that "can evaluate candidate skills and experience working and managing a diverse and inclusive workplace" (City and County, 2020, p. 17).

\section{Policies and Trainings}

- Workplace Bullying: "In this training, participants learn how to identify workplace bullying, as well as skills, strategies, and actions to take to stop workplace bullying" (City and County, n.d.-h).

- Policy on Language Diversity: “The City's Policy on Language Diversity recognizes that a workforce that speaks languages other than English enhances the services provided to the City's culturally diverse public by providing efficient and accessible public services to its non-English speaking communities. An employee's use of a language other than English is not only an asset in the provision of public services but, with few exceptions, is a legally protected right" (City and County, n.d.-h).

- Policy Regarding the Treatment of Co-workers and Members of the Public: "City policy requires employees to treat co-workers and members of the public with courtesy and respect. City employees and managers are responsible for maintaining a safe and productive workplace which is free from inappropriate workplace behavior" (City and County, n.d.-h).

\section{Conflict Resolution}

\section{Improving Work Experience of Black and LatinX}

Since improving the work-life experience of the Black and LatinX CCSF workforce was identified as an area of concentration for DHR's Racial Equity Action Plan, several initiatives have either been started or are being planned to help in creating a more inclusive working environment for this population.

- Improving response time and intervention strategies when allegations of mistreatment are brought to DHR's attention (City and County, 2020, p. 3).

- Citywide Peer Mediation Program: "Interpersonal conflict between employees that does not constitute an Equal Employment Opportunity complaint will be eligible for the Peer Mediation Program. The program is voluntary and aims to promote positive, healthy work. environment through a facilitated problem-solving process. The participants (with support of mediators) will develop and agree upon resolutions to conflict. Peer Mediators will be City employees selected through a volunteer application process and trained on workplace mediation, prior to hosting any mediation sessions" (City and County, 2020, p. 50).

- In November 2020, the Mayor announced that "DHR had commissioned an independent and comprehensive review of the City's workplace policies and practices with a focus on claims of bias, harassment, discrimination, and retaliation, led by Stanford Law School Professor Emeritus William B. Gould IV" (City and County, 2020, p. 4).

- DHR proposes to establish a "dedicated function for review and oversight of proposed discipline, with the primary focus on the initial due process procedures and on ensuring consistency in the imposition of major 
discipline (defined as suspensions greater than 10 days and terminations, including "last chance" agreements)"

(City and County, 2020, p. 4).

- Lead by DHR's Workforce Development Team, DHR will continue to improve its 24 Plus Performance

Management Program for its supervisors, including training for equitable and compassionate discipline and separation (City and County, 2020). On FY21-22, DHR will augment this training, emphasizing expectation setting, coaching, performance management, conflict management, and equitable discipline and corrective action (City and County, 2020, p. 34).

- For FY22-23: DHR will "expand the curriculum of training and resources for Skelly officers. Enhance training curriculum to provide an emphasis identification of race-based bias in proposed disciplinary actions" (City and County, 2020, p. 35).

\section{SPECIAL EMPLOYEE BENEFITS}

Employee Assistance Program (EAP)
The county has its Employee Assistance Program "offering a variety of confidential counseling, consultation, coaching \& education services. EAP counselors are licensed therapists with many years of diverse experience in mental health, business and as City employees" (San Francisco Health, 2021).
Additional Employee Leaves

\section{Child Bonding Leave, Family Care Leave, and Parental Leave (City and County, n.d.-i).}

One of the action items in the Racial Equity Action plan is to "set up processes and open communication channels, so management is available to respond to employees' non-work-related needs that contribute to the overall work quality. Center the most vulnerable individuals. e.g., transportation stipends, exercise stipends, childcare, etc. FY21-22: Study the benefits and feasibility of a citywide program for alternative work schedules and flexible time. Research current City departments' flexible time arrangements to better understand which employees are using flexible schedules and how they are using them. FY22-23: Create guidance for supervisors and human resource professionals on best practices in providing flexible and alternative work schedules consistently and transparently" (City and County, 2020, p. 45). 


\section{Workforce Representation Analysis - San Francisco}

The following data is an analysis of the ethnicity of the City and County of San Francisco's 2020 workforce compared to the City's overall population and the current labor market available citywide.

\section{$\underline{\text { Representation: }}$}

- In 2020 Asian (39.80\%) employees have the highest representation among the four major ethnicities, followed by White (28.57\%), then by Black or African American (15.13\%), and Hispanic or Latino $(15 \%)$ close together.

- The employee groups that exceeded their citywide demographic representation are Black or African American, exceeding by $9.53 \%$, and Asian, exceeding by $3.8 \%$. The employee representation below their population demographic is white, which is lower by $11.63 \%$, and Hispanic or Latino, which is lower by .20\%.

- For the Officials and Administrators job category, both African American and White employee groups are overrepresented. On the one hand, Hispanic and Asian employee groups are both underrepresented.

\section{Utilization:}

- The City's employee representation that is roughly equal to or exceeds the available workforce are the following employee groups: Black or African American exceeding by $11.3 \%$, Asian exceeding by $8.02 \%$ and Hispanic or Latino exceeding by .44\%. White employees in the City and County of San Francisco are underrepresented by $16.97 \%$.

- In terms of representation in leadership positions, specifically for the City's Officials and Administrators, the white employees have the highest representation among the four major ethnicities though they are slightly underrepresented as compared to the available supply of officials and administrators in the City.

- Among the three non-white employee groups, Black or African American is the only group that exceeded its available supply of officials and administrators.

\section{CITY AND COUNTY OF SAN FRANCISCO WORKFORCE}

County Population Estimate County Labor Market County Employees
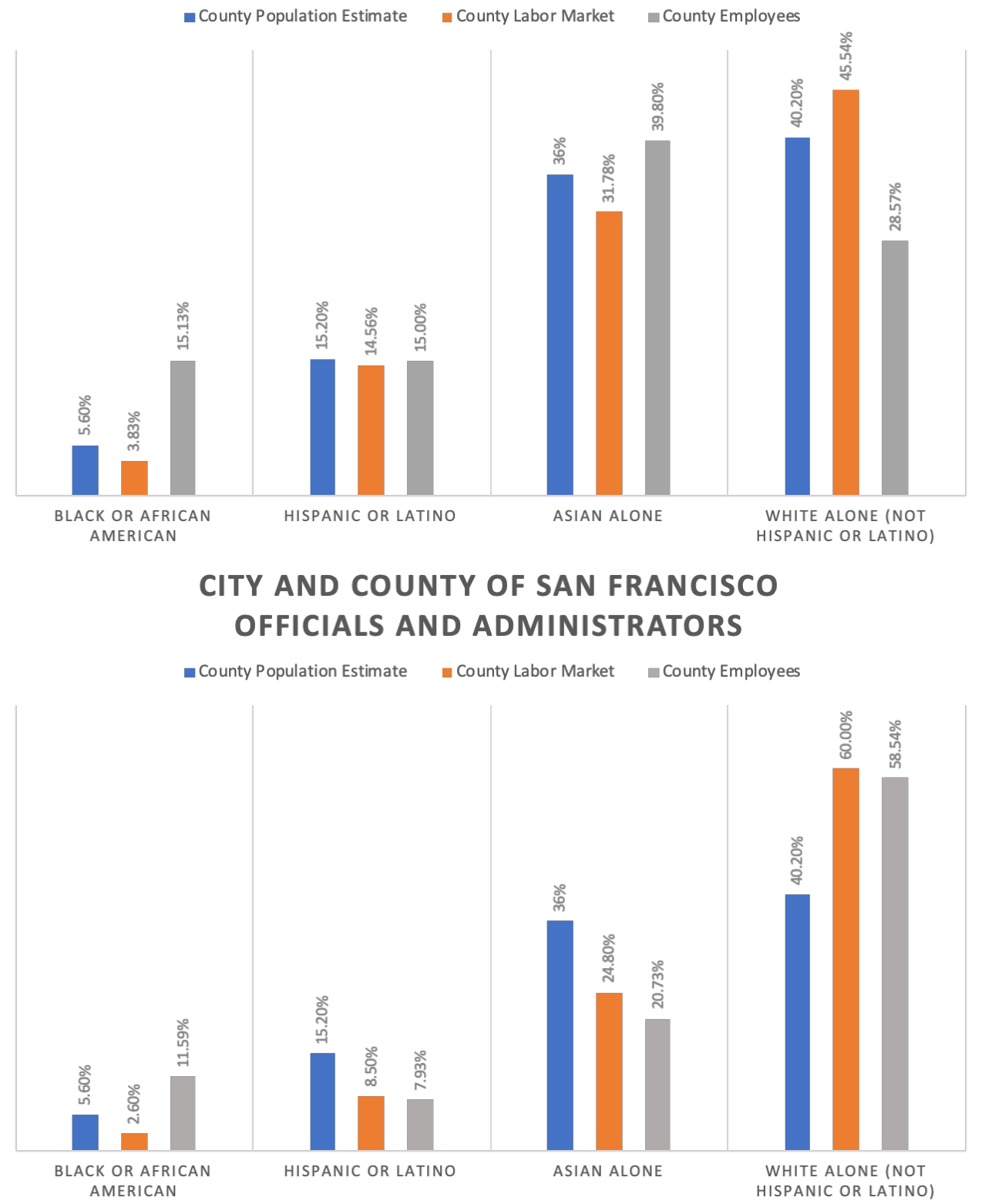


\section{County of San Mateo}

The County of San Mateo is located southwest of San Francisco, and its jurisdiction covers most of the San Francisco Peninsula. This region has an approximate population size of 766,573 (US Census Bureau, 2021). As of 2021, the county organization has a total workforce of 6,882 employees (S. Trela, personal communication, April 19, 2021).

The motion to create the Diversity and Inclusion (D\&I) Strategic Initiative was approved by the county's Board of Supervisors on August 8, 2017 (County of San Mateo, 2017a). The action steps of this initiative are contained within San Mateo’s 2018-2021 Equal Employment Opportunity Plan (EEOP), which serves as the countywide blueprint of strategies aimed at promoting diversity, inclusion, and equal employment opportunity across its organization (County of San Mateo, 2017b). This plan also details the progress of diversity-friendly initiatives developed and implemented between 2014 and 2017 (County of San Mateo, 2017b). This plan is a collaborative work between members of the Equal Employment Opportunity Advisory Committee, composed of members appointed by department heads, and with the guidance of the Human Resources Department, particularly the Equal Employment Opportunity unit (County of San Mateo, 2017b). The purpose of EEOP is to "reaffirm the County's commitment to equal employment opportunity and diversity and inclusion in its employment practices, program operations, and service delivery systems" (County of San Mateo, 2017b, p. 5). This plan also highlights the importance of having county leadership support to guarantee the plan's success (County of San Mateo, 2017b). The overarching goals of this plan are to:

- Attract and retain a diverse workforce that is reflective of the community;

- Increase inclusion among employees of differing identities and backgrounds to promote a welcoming and productive workforce that responds to the needs of a diverse community; 
- Promote a work environment that is free from all forms of discrimination and harassment, including unfair treatment based on sex, pregnancy, childbirth or related medical conditions, race, veteran status, religion, color, national origin or ancestry, physical or mental disability, medical condition, marital status, age, gender (including gender identity and gender expression), sexual orientation, use of family medical leave, genetic testing, or any other basis protected by federal or state law;

- Provide opportunities for career development and advancement for all employees, and identify barriers and challenges to diversity and inclusion and remove them or develop solutions, and;

- Increase the pool of qualified, diverse applicants, particularly for job categories or areas that are underrepresented. (County of San Mateo, 2017b, p. 5)

The Diversity and Inclusion (D\&I) Strategic Initiative is a multi-phased and multi-year effort that was initially piloted in three to four departments and divisions with the intent to "reinforce D\&I as a core county value and business goal to better serve an increasingly diverse workforce" (County of San Mateo, 2017b, p. 41). The core goals and actions that these departments intend to accomplish in phases, overtime, are as follows:

- Assess and learn about department workforce D\&I priorities, needs, challenges, and goals

- Conduct online employee surveys and obtain input from advisory groups, leadership, and stakeholders;

- Identify challenges and barriers to D\&I to address them and/or develop solutions;

- Review, revise, or develop policies and procedures that remove barriers and/or advance D\&I goals;

- Attract and retain a diverse workforce that is reflective of the community; 
- Promote proactive measures by assisting departments to develop and implement D\&I Action Plans;

- Develop and deliver unconscious bias training for hiring managers and supervisors, and;

- Review or develop other D\&I action steps and training to foster compliance, implementation, and advancement of D\&I goals. (County of San Mateo, 2017b, p. 41)

Phase 1 will focus on assessment and learning (Fall 2017-2018 and ongoing), Phase 2 will develop and implement D\&I action plans, and Phase 3 will promote diversity and inclusion at all levels of the organization, both occurring in 2018-2019 and ongoing. Lastly, phase 4 will evaluate diversity and inclusion metrics and goals (2019 and ongoing) (Park, 2017).

Drivers of Creating the Plan: The need to develop a diversity and inclusion strategic plan is due to the rapid change of the demographic composition of San Mateo County (Park, 2017). Currently, minority groups are considered the majority in San Mateo County, compared to its 1990 census, where the majority was white at 72\% (Park, 2017). The County of San Mateo is considered to be one of the most diverse counties in the state (Vaillancourt, 2017). Additionally, the county recognizes the benefits of workforce diversity to organizational efficiency. According to Donna Vaillancourt, Human Resource Director, diversity and inclusion "is a business imperative for any organization that wants to work smart, innovate and promote equity" (Vaillancourt, 2017). Despite the different challenges that diversity brings, the county believes that the rewards of diversity are greater than its challenges (Park, 2017).

In addition, the county recently promoted its health equity officer to the newly created Chief Equity Officer position, whose role is aimed at "bettering the welfare and outcomes of both employees and residents" through the lens of equity and inclusion (County of San Mateo, 2021, 
p. 1). This research, however, was not able to confirm the Chief Equity Officer's oversight on the EEO plan or whether a different racial equity plan is underway. 


\section{Diversity Management Program - County of San Mateo}

BP 1: The development of a formal diversity process that is contained in laws, rules, or procedures. Both human and financial time and resources are devoted to the program. In best practice organizations, diversity is a process that is an integrated, ongoing, and measurable strategy.
The proposed creation of the County of San Mateo's Diversity and Inclusion Strategic Initiative was accepted and affirmed by all five Board of Supervisors during the August 8, 2017 board meeting (County of San Mateo, 2017a). The formal diversity process and strategic plans were established as part of the adoption of the county's Equal Employment Opportunity Plan (County of San Mateo, 2017b). Stated in this plan is the Board's full and active support in the plan's objectives and the approval of resources and budgetary assistance needed towards diversity and inclusion efforts by county departments (County of San Mateo, 2017b).
BP 2: Diversity efforts are primarily decentralized, with a central governing body outlining the requirements of the plans with individual agencies and departments developing their own plans that are tailored to their specific needs. This reinforces a sense of ownership and ensures that managing diversity has both top-level support and is a reality throughout the organization.

Although there is no clear intent of decentralizing or giving authority to each department to develop each of their diversity plans, the Board of Supervisors has stated clear responsibilities in the plan and assigned them to several officials and members of its workforce. The County Manager will be responsible for "providing executive leadership and oversight to ensure that department heads take action as necessary to achieve and implement the plan's goals and objectives" (County of San Mateo, 2017b, p. 7). The Human Resource Director is responsible for the "general administration of the Plan and will review personnel policies, employment practices, and procedures, and make recommendations on steps to ensure equal employment opportunity (County of San Mateo, 2017b, p. 7). The department heads "will adhere to the County's Equal Employment Opportunity Plan, and are responsible for achieving progress toward the goals and objectives of the Plan in their departments" (County of San Mateo, 2017b, p. 7). Department heads will ensure that decisions in employment are "consistent with the County's personnel practices and equal employment opportunity principles" (County of San Mateo, 2017b, p. 7) and are expected to collaborate with the EEO manager in identifying departmental challenges and obstacles and working with the managers in finding solutions to issues (County of San Mateo, 2017b). The Equal Employment Opportunity Manager will collaborate with management, department heads, and employees in developing and implementing policies, problem-solving and progress monitoring of the EEO Plan (County of San Mateo, 2017b). Equal Employment Opportunity Advisory Committee are select employees from different Departments who will serve as the plan's resource and advisory group (County of San Mateo, 2017b). Finally, the plan also mentions the responsibility of managers, supervisors, and county employees to take proactive steps to adhere to the EEO policies and plans (County of San Mateo, 2017b).

BP 3: In best-practice organizations, diversity training is provided to the workforce. The training is not limited to managers but is extended
Some of the EEO accomplishments between 2014 to 2017 that are mentioned in the plan with regards to diversity training include: 
throughout the workforce. Successful organizations incorporate diversity into mentoring efforts, leadership training, and management-byresults programs

- "Added requirement that all new supervisors and managers participate in 3-hour, instructor-led, in-person training on EEO Policy and diversity/ inclusion at the County of San Mateo, within 24 months of becoming a supervisor or manager" (County of San Mateo, 2017b, p. 38).

- "Provided more than 50 department-specific and other training classes, covering approximately 1,200 employees, in the areas of diversity and EEO” (County of San Mateo, 2017b, p. 38).

Under the Diversity and Inclusion Strategic Initiative, some of the diversity training goals mentioned include the following:

- "Develop and deliver unconscious bias training for hiring managers and supervisors" (County of San Mateo, 2017b, p. 41).

- "In collaboration with departments, the Human Resources Training Division will continue to offer and/or promote training and educational opportunities such as: Diversity and related training, including offerings related to: multiple generations in the workforce, cultural awareness and communications, conflict resolution, and inclusion issues" (County of San Mateo, 2017b, p. 42).

Additional training that is provided with the help of the Human Resources Training Division includes "specialized trainings on diversity, equity, and inclusion on topics such as cultural competency, diversity/EEO/ inclusion, equity, implicit bias, transgender policy and gender identity/ pronouns, and related topics" (County of San Mateo, 2020, p. 4).

BP 4: Best practice

organizations utilize workforce data and demographics to compare statistics reported for the civilian labor force. Occupations with underutilization are identified, and goals are established to reduce the underutilization. All of the best-practice organizations add creative and innovative recruitment strategies that get results and set their programs apart.
The December 2020 EEO Utilization Report, which contains San Mateo County's workforce utilization data, has indicated that the county is "actively working to increase representation of all historically underutilized groups" and that it will "continue to explore ways to recruit and retain employees of underrepresented gender, race/ethnicity groups, and other EEO categories" (County of San Mateo, 2020, p. 3). The listed objectives and steps towards this goal that pertain to recruitment efforts include (1) ensuring equality of opportunity in hiring, recruitment, and succession planning; (2) continuing to increase recruitment and outreach to attract a diverse applicant pool; and (3) providing equal opportunity to candidates during the recruitment and selection process (County of San Mateo, 2020, p. 4).

Contained in both the EEO Utilization Report and EEO Plan are the following recruitment and selection strategies:

- "Review employment data to identify barriers to attracting a diverse applicant pool" (County of San Mateo, 2020, p. 4).

- "Participation in career and job fairs, expos, and community events, and targeted advertising of job opportunities to professional organizations representing women, minority, and other groups" (County of San Mateo, $2017 \mathrm{~b}, \mathrm{p}$. 38). Also included is continued partnership with local educational institutions/colleges (County of San Mateo, 2020). 
- "Expanded internship programs including Jobs for Youth to provide opportunities for community youth from a variety of backgrounds to learn about potential careers, including those in government service" (County of San Mateo, 2017b, p. 38).

- Management Fellows Program: A formalized county fellowship program to broaden the pool of qualified applicants and bolster the talent pipeline with candidates of diverse backgrounds. This program gives master's graduate students opportunities to work on meaningful projects within the county for up to two to three years (County of San Mateo, n.d.-a).

- "Expanded STEP (Supported Internship and Employment Program) to a year-round program, providing additional opportunities for the community's foster youth to expand work-related knowledge through jobreadiness training, job shadowing, employment coaching, and hands-on work experience in a County department" (County of San Mateo, 2017b, p. 38).

- "Used social media to enhance recruitment efforts, including Facebook, LinkedIn, and Twitter as a means to communicate promotional information to applicants and employees" (County of San Mateo, 2017b, p. 38).

- "Expanded online candidate testing for employment opportunities, reducing barriers caused by need for transportation to County facilities, which may be particularly challenging for candidates with disabilities, children or other family responsibilities, or candidates of lower socio-economic status" (County of San Mateo, 2017b, p. $38)$.

- "Continue to review and evaluate hiring processes, working to identify and remove artificial barriers to employment, including by reviewing interview questions and tests to ensure they do not have an improper adverse impact" (County of San Mateo, 2020, p. 4).

- "Provide recruitment guidance to hiring managers and encourage diverse interview panels" (County of San Mateo, 2020, p. 4).

- Additionally, Human Resources senior management and supervisory staff will be provided with the EEO Utilization Report to be made aware of the EEO objectives (County of San Mateo, 2020, p. 5).

BP 5: Best practice agencies have found that requiring diversity programs through the law, executive order, or other mandates compels agencies to establish serious goals and to make earnest efforts toward meeting those goals.
The Equal Employment Opportunity Plan recognizes that its success is dependent upon the support from the county's leadership and the commitment from its department heads, managers, supervisors, and employees; therefore, the Board of Supervisors has assigned specific responsibilities to each of these entities (County of San Mateo, 2017b).

\section{BP 6: Best practice} organizations have established a review committee responsible
As stated in the EEO Plan, department heads will appoint members within their team to serve as part of the countywide Equal Employment Opportunity Advisory Committee. On the EEO Advisory Committee website, it states that "the Committee will consist of no more than 15 members appointed by the County Manager, and 3 liaisons (Commission on 
for establishing policies, providing technical assistance, reviewing/approving plans, and monitoring progress toward achieving goals.

the Status of Women, Civil Service Commission and Commission on Disabilities)" (County of San Mateo, n.d.-b).This committee functions as a resource group "on equal employment opportunity matters, initiatives, and programs" and "advises and recommends courses of action regarding the equal employment opportunity plan, and diversity and inclusion initiatives, efforts, and programs" (County of San Mateo, 2017b, p. 9). The Committee will:

- "Serve as a committee to review, learn, collaborate, and share best practices and recommendations on equa opportunity and diversity and inclusion matters, programs, policies, and initiatives" (County of San Mateo, 2017b, p. 9);

- $\quad$ "Be familiar with the County's employment policies and procedures, resources, trainings, and relevant laws pertaining to equal employment opportunity matters" (County of San Mateo, 2017b, p. 9);

- "Identify and assess department priorities, challenges, obstacles, goals, and initiatives aimed at fostering diversity and inclusion and equal opportunity, and"(County of San Mateo, 2017b, p. 9);

- "Develop solutions and best practices to address and improve policies, practices, and challenges" (County of San Mateo, 2017b, p. 9).

- The EEO Advisory Committee will monitor, review, and make recommendations on issues related to the successful implementation of the plan, such as organizations and resources to administer an effective EEO program; recruitment and retention; classification; selection and related personnel activities; upward mobility; and, program understanding and support (County of San Mateo, n.d.-b).

In developing and approving plans and technical assistance, these responsibilities are assigned to the county's Equal Employment Opportunity Manager. Some of the specific duties assigned to the manager include:

- "Partnering with management, department heads, and employees to develop and implement policies and practices that foster an inclusive and productive workplace" (County of San Mateo, 2017b, p. 8);

- "Assisting management and department heads in identifying and resolving problem areas related to the County's Equal Employment Opportunity Policy and Plan" (County of San Mateo, 2017b, p. 8);

- "Monitoring the effectiveness of the County's Equal Employment Opportunity Plan, as well as individual departmental efforts, through statistical and other means" (County of San Mateo, 2017b, p. 8);

- "Acting as a technical resource in the area of equal employment opportunity programs and policies" (County of San Mateo, 2017b, p. 8);

- "Providing technical assistance to the Equal Employment Opportunity Advisory Committee regarding policies, procedures, and resources available" (County of San Mateo, 2017b, p. 8);

BP 7: Effective diversity programs also link recruitment, development, and retention strategies to organizational performance. They integrate
The County of San Mateo has been providing various career development, retention, and succession planning strategies that continuously helps its workforce towards their career growth and advancement since 2014 . 
employee development processes and map career paths to see what critical skills are necessary to advance, then communicate these skills to employees and provide training.
- $\quad$ "Robust performance management system (CPMS) that links work tasks to the County vision, provides employee input for avenues of expansion of duties/exposure, and includes career development goal planning" (County of San Mateo, 2017b, p. 39).

- "Encouraged departments to use work out of class opportunities, training, and promotions from within as a means to provide equal opportunities" (County of San Mateo, 2017b, p. 39).

- "Create, sponsor and/or support educational diversity events, speakers, panels, and/or programs that will offer additional opportunities to employees to gain experience and knowledge about jobs and career paths" (County of San Mateo, 2017b, p. 42).

- "An Executive Leadership Academy to train and identify managers for promotion to Director, Assistant Director, Deputy Director, and similar positions" (County of San Mateo, 2017b, p. 40).

- "A comprehensive Supervisory Academy for front line supervisors" (County of San Mateo, 2017, p. 40).

- "A Management Development Program, to continue the professional development of managers and help prepare employees to assume high-level leadership positions"(County of San Mateo, 2017b, p. 40).

- "The Management Talent Exchange Program (MTEP); which allows aspiring managers in local governments within San Mateo and Santa Clara counties the opportunity to develop skills and competencies, gain valuable hands-on experience, and build relationships through participation in a 3-month exchange in another government agency" (County of San Mateo, 2017b, p. 40).

- "The Public Sector Leadership Academy, which provides information to management employees about innovative concepts and practices in the public sector, with topics including public policy, employee engagement, trends, and ethics"(County of San Mateo, 2017b, p. 40).

- "A Lead Worker Academy, designed for current and future lead workers, to introduce the core responsibilities for working in a lead role, including sessions on effective communication and career growth" (County of San Mateo, 2017b, p. 40).

- $\quad$ "The Stepping Up and Leading Up Academy to help line employees identify and explore career goals and develop leadership, communication, and other relevant skills" (County of San Mateo, 2017b, p. 40).

- "In collaboration with departments, the Human Resources Training Division will continue to offer and/or promote training and educational opportunities such as: Degree and certificate programs and customized departmental training programs" (County of San Mateo, 2017b, p. 42).

- The D\&I Strategic Initiative also intends to "focus on additional career development and succession planning strategies such as acting assignments, temporary/provisional appointments, job rotation, short term projects, committee assignments, enhance training opportunities, development experience opportunities, coaching, and mentoring project, retaining institutional knowledge, exploring enhanced online technology as a cost-effective way to open doors for training and educational opportunities, and sponsoring Career-Development Assessment Centers for employees interested in growing and preparing for promotional opportunities" (County of San Mateo, 2017b, p. 42). 
BP 8: Accountability for the results of diversity programs is another attribute of best-practice organizations. Accountability is determined through metrics, surveys, focus groups, customer surveys, management, employee evaluations, and training and education evaluations.
At the August 8, 2017 Board of Supervisors meeting, it was mentioned that phase 4 of the plan would evaluate diversity and inclusion metrics and goals (County of San Mateo, 2017a).

\section{UNIQUE DIVERSITY MANAGEMENT STRATEGIES}

Diversity STARS Award Program: This program "recognizes outstanding County programs that demonstrate success in fostering diversity and inclusion, enhancing services or support for underrepresented or at-risk groups or communities, increasing cultural awareness and understanding, and/or creating a welcoming, inclusive workplace or program"(County of San Mateo, 2017b, p. 39).

\section{SPECIAL EMPLOYEE BENEFITS}

Employee Assistance Program In addition to "work-life assistance to help employees and their family members manage problems related to work, (EAP) personal relationships, stress, finances, substance abuse, and other life concerns" the county's EAP program also offers "job performance referrals, management consultations, critical incident debriefings, and training programs to enhance workplace health and employee effectiveness" (County of San Mateo, n.d.-c).

\section{Other Benefits}

"Encouraged departments to offer flexible schedules, Voluntary Time Off (VTO), telecommuting, and other alternatives to maximize employees work/life balance, including child care, parental and sibling care, health recovery, education opportunities, and better commute alternatives (recognizing the high percentage of employees that live more than 30 minutes away from their county worksite)" (County of San Mateo, 2017b, p. 39). 


\section{Workforce Representation Analysis - San Mateo}

The following data analyzes the ethnicity of the County of Mateo's 2021 workforce compared to the county's overall population and its countywide available workforce.

Representation:

- For 2021, white (27.60\%) employees had the highest representation among the four major ethnicities, followed closely by Asian $(24.60 \%)$ and Hispanic/Latino $(21.80 \%)$, then by Black or African American (4.90\%).

- The only employee representation that exceeded their countywide population is the Black or African American group exceeding $2.1 \%$. The rest of the three major ethnicities are underrepresented: white, which is lower by $11.10 \%$, Asian (6\%), and Hispanic or Latino (2.2\%).

- For the Officials and Administrators job category, both African American and White employee groups are overrepresented. Hispanic and Asian employee groups, on the other hand, are both underrepresented.

Utilization:

- Similar to the outcome on population representation, the Black or African American employee group is the only one that exceeded their available countywide workforce by $2.8 \%$. The rest of the three major groups are underutilized by $2.29 \%$ (Hispanic), $5.34 \%$ (Asian), and $11.50 \%$ (white).

- In terms of utilization in leadership positions, white employees $(41.40 \%)$ have the highest representation among the four major ethnicities. Still, they are underutilized compared to its available supply of officials and administrators within the county.

- Among the three non-white employee groups, Asian is the only underutilized group (-7.8\%). Black or African American and Hispanic or Latino have both exceeded the percentage of its available supply of officials and administrators.

\section{COUNTY OF SAN MATEO WORKFORCE}

- County Population Estimate $\quad$ County Labor Market $\quad$ County Employees

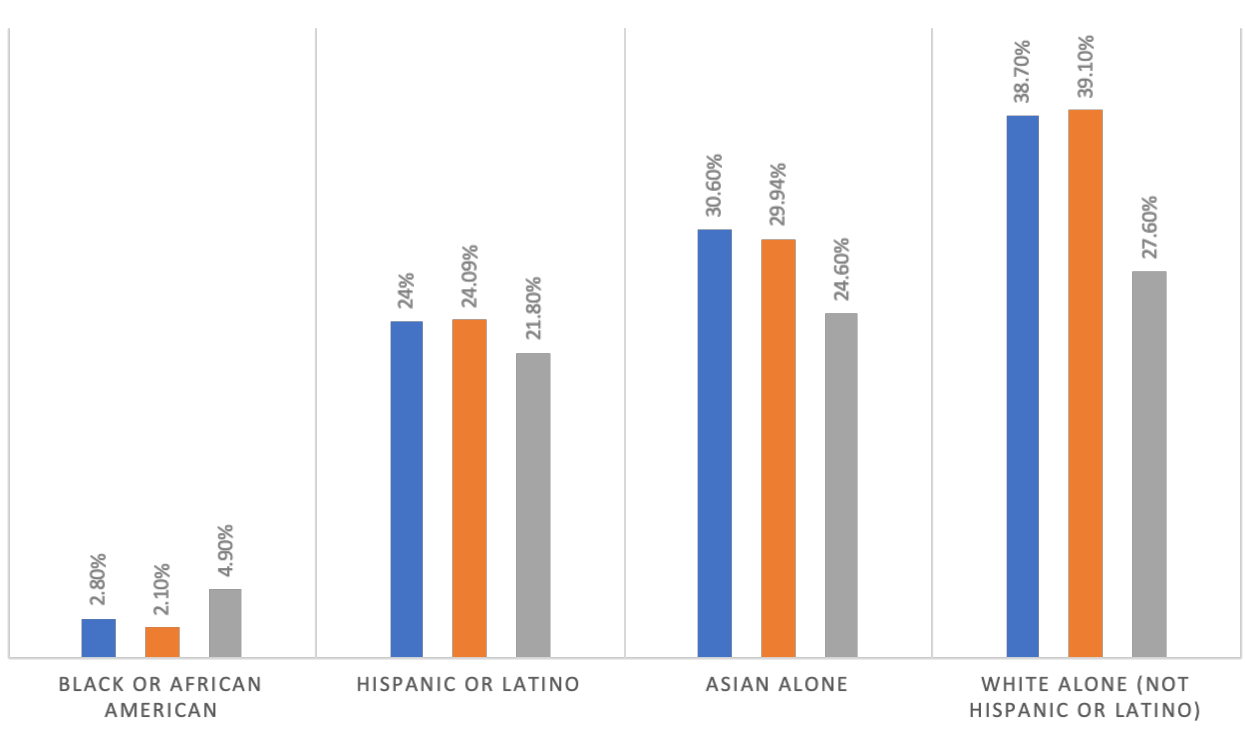

\section{COUNTY OF SAN MATEO OFFICIALS AND ADMINISTRATORS}

- County Population Estimate - County Labor Market $\quad$ County Employees

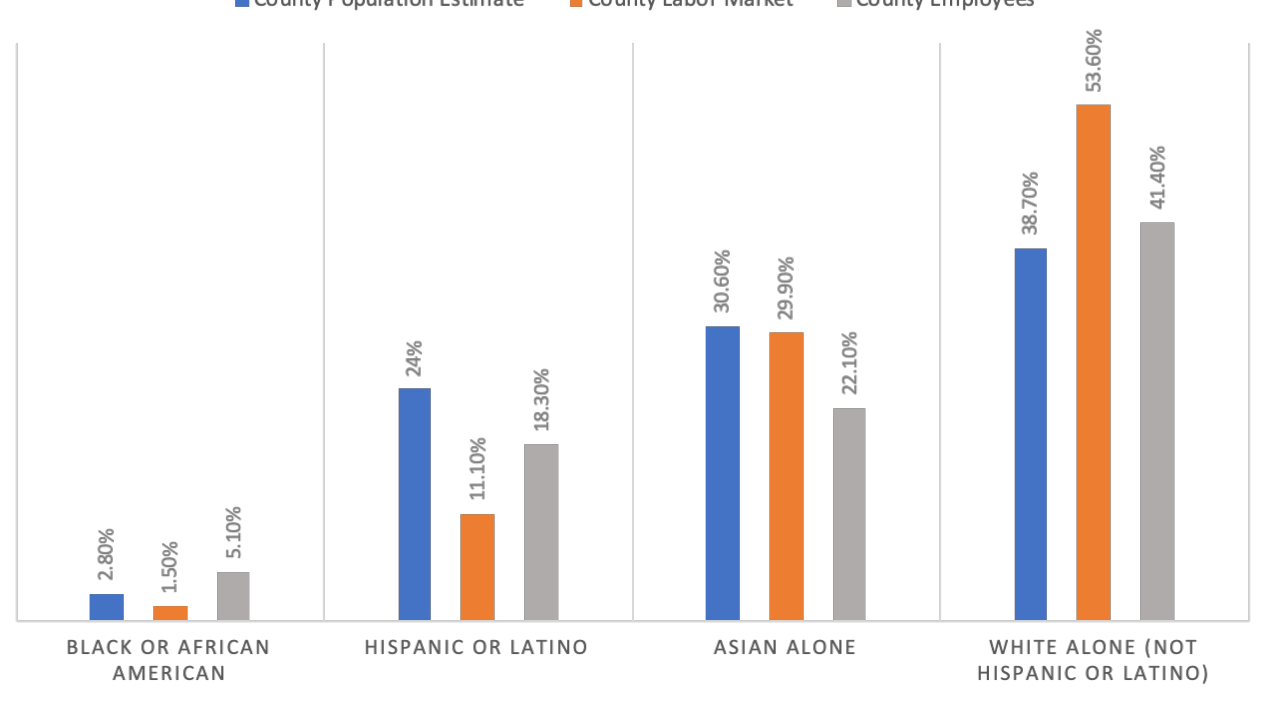




\section{County of Sonoma}

The County of Sonoma is located in the northern part of the Bay Area region and is also known for being the largest wine producer in California's Wine Country. This region has an overall population size of approximately 494,336 (US Census Bureau, 2021). As of January 2021, the county organization had a total workforce of 3,855 employees (V. Willard, personal communication, February 5, 2021).

The County of Sonoma's diversity management programs are embedded in its 5 Year Strategic Plan 2021-2026, which was approved by its Board of Supervisors on March 2, 2021 (County of Sonoma, 2021a). The county's diversity goals and objectives are contained within its Racial Equity and Social Justice pillar, which is one of the five distinct pillars or foci of this plan (County of Sonoma, 2021b). It is said that "Sonoma County's collective well-being and prosperity are impacted by significant racial inequities" and that by "focusing on racial equity and social justice in the Strategic Plan, the Board of Supervisors can begin to institutionalize equity and address disparate impacts on people of color, both internally as an organization and in the community" (County of Sonoma, 2021b, p. 12).

The goals of the Racial Equity and Social Justice Strategic pillar are: "fostering a County culture that supports the commitment to achieving racial equity; implementing strategies to make the County workforce reflect County racial demographics across all positions; ensuring racial equity throughout all County systems and services; and, engaging the key community and internal stakeholders in developing priorities to advance racial equity" (County of Sonoma, 2021b, p. 12-13). 
Sonoma County's stated mission is "to enrich the quality of life in Sonoma County through superior public services" (County of Sonoma, 2021b, p. 2). And tied in with this mission is the value of collaboration or "ensuring that the right people are being included in the discussion" (County of Sonoma, 2021b, p. 3). Hence, the development of the 5 Year Strategic Plan did not only seek the input of the county workforce, but it also requested community stakeholders and the public to take part in its creation (County of Sonoma, 2021). This effort generated input from over 1,700 respondents through “online surveys, focus groups, open house sessions, community meetings, stakeholder presentations, letters and emails" (County of Sonoma, 2021b, p. 1).

The goals and objectives of the 5 Year Strategic Plan will be a shared oversight between the Human Resources Department and the newly created Office of Equity, which was established in August 2020 (County of Sonoma, 2021b).

In addition, the County of Sonoma is one of the several counties that had sent staff to join the Government Alliance on Race and Equity (GARE). The county sent 12 of its staff from different departments. From this cohort, the county formed Sonoma County Racial Equity Alliance and Leadership (SoCoREAL) (County of Sonoma, 2021c). The Office of Equity was established through the help of SoCoREAL and SoCoLERN (the LatinX Employee Resource Network) (County of Sonoma, 2020).

Drivers of creating the plan: Over the last several years, there has been a trend in organizations to prioritize diversity management (C. Cramer, personal communication, February 5, 2021). Public agencies, particularly local governments, have become more aware of their effect on equitable outcomes with their services and policies in their communities. The human 
resources profession has also become more aware of the benefits of a diverse workforce, particularly in public organizations. Concurrently, local LatinX leaders (Sonoma County's diverse population primarily consists of Latinos) have been pushing the county's Board of Supervisors to focus on the value of equity to support improved outcomes in the county's Latino population. Due to these factors, the county is incorporating diversity management into its strategic plans (C. Cramer, personal communication, February 5, 2021). 


\section{Diversity Management Programs - County of Sonoma}

BP 1: The development of a formal diversity process that is contained in laws, rules, or procedures. Both human and financial time and resources are devoted to the program. In best practice organizations, diversity is a process that is an integrated, ongoing, and measurable strategy.

BP 2: Diversity efforts are primarily decentralized, with a central governing body outlining the requirements of the plans, with individual agencies and departments developing their own plans that are tailored to their specific needs. This reinforces a sense of ownership and ensures that managing diversity has both top-level support and is a reality throughout the organization.
An Office of Equity was established in August 2020, and the County of Sonoma's 5-Year Strategic Plan was adopted. Both require human and financial time and resources to execute. The goals and objectives stated in the 5-Year Strategic Plan are meant to be integrated, ongoing, and measurable.

\section{BP 3: In best-practice} organizations, diversity training is provided to the workforce. The training is not limited to managers but is extended throughout the workforce. Successful organizations incorporate diversity into mentoring efforts, leadership
The Office of Equity and Human Resources Department will serve as the central governing body. However, it is yet to be determined if departments will be allowed to develop their programs (C. Cramer, personal communication, February 5 , 2021).

One of the goals of the Racial Equity and Social Justice pillar is to "foster a County organizational culture that supports the commitment to achieving racial equity" (County of Sonoma, 2021b, p. 12). And one of the ways that the county is doing this is by investing in the "ongoing and continually developing racial equity learning program, including understanding the distinction between institutional, structural, interpersonal, and individual racism, for County leadership and staff” (County of Sonoma, 2021b, p. 12). This will be a joint oversight between the Office of Equity and the Human Resources Department (County of Sonoma, 2021b, p. 12). 
training, and management-byresults programs.

BP 4: Best practice organizations utilize workforce data and demographics to compare statistics reported for the civilian labor force.

Occupations with underutilization are identified, and goals are established to reduce the underutilization. All of the best-practice organizations add creative and innovative recruitment strategies that get results and set their programs apart.
Goal number two of the Racial Equity and Social Justice pillar is to "implement strategies to make the County workforce reflect County demographic across all levels" (County of Sonoma, 2021b, p. 13). And the two objectives that are tied into this goal are (1)“identify opportunities to enhance recruitment, hiring, employee development, and promotional processes to reflect the value of having the perspectives of people of color represented at all levels in the County workforce" (County of Sonoma, 2021b, p. 13). This objective is a shared responsibility between the Office of Equity and Human Resources (County of Sonoma, 2021b, p. 13). (2) "Implement countywide strategies to recruit, hire, develop, promote and retain County employees of color, produce an annual report card assessing progress, and update strategies as needed" (County of Sonoma, 2021b, p. 13). This is an oversight by Human Resources (County of Sonoma, 2021b, p. 13). It has also been said that reports are currently being developed, and a plan on doing data analysis before opening recruitment is in the works. More data sharing with the departments are also planned (C. Cramer, personal communication, February 5, 2021).

Additionally, a hiring toolkit is getting ready to be released that highlights hiring best practices with a focal point on ways to eliminate bias and promote equity in the hiring processes to achieve a more diverse workforce (C. Cramer, personal communication, February 5, 2021).
BP 5: Best practice agencies have found that requiring diversity programs through the law, executive order, or other mandates compels agencies to establish serious goals and to make earnest efforts toward meeting those goals.
Sonoma County's 5-Year Strategic Plan is a board-approved mandate. "Each objective in the Strategic Plan has been assigned lead, co-lead, and participating departments. It will be up to those departments to identify the implementation plan required to achieve each objective. The goal is to achieve each objective by the end of 2026. However, the timeline for implementation will vary based on many factors, including the complexity of the objective, partner and community input, workforce capacity and availability, budget availability, and effective coordination with County partners.

Implementation plans will be reviewed regularly to assess their success in meeting the milestones set out in the objectives and will be embedded within department work plans and budgets, as appropriate" (County of Sonoma, 2021b, p. 2).

\section{BP 6: Best practice} organizations have established a review committee responsible for establishing policies, providing technical assistance, reviewing/approving plans, and monitoring progress toward achieving goals.
Part of goal 1 of the Racial Equity and Social Justice pillar is to help in fostering a racially equitable organization is by creating a racial equity core team "to advance equity initiatives across all departments in collaboration with the Office of Equity" (County of Sonoma, 2021b, p. 2). Their purpose will be to guide departments and establish an equity plan for the county to follow (C. Cramer, personal communication, February 5, 2021). The Office of Equity has successfully recruited a total of 23 equity ambassadors out of the 26 different departments that would form this team (County of Sonoma, 2021d). 
BP 7: Effective diversity programs also link recruitment, development, and retention strategies to organizational performance. They integrate employee development processes and map career paths to see what critical skills are necessary to advance, then communicate these skills to employees and provide training.
Although not a part of the Racial Equity and Social Justice Pillar, one of the pillars tuned to organizational performance is the Organizational Excellence Pillar. It is said that "in order to serve Sonoma County residents and fulfill State and Federal mandates, the county organization must perform at its best...employees must be engaged and have job satisfaction so they are inspired to provide excellent service. The County's workforce should reflect the County's demographic makeup so it can best meet the needs of our diverse community" (County of Sonoma, 2021b, p. 9).

One of the goals under the Organization Excellence Pillar is for the County of Sonoma to "become an employer of choice with a diverse workforce that reflects its community" and to be an "employer with a positive work culture that builds engaged and developed employees" (County of Sonoma, 2021b, p. 11). One of the objectives to help this goal is by supporting "employee professional growth and retention by investing in high-quality training, development, and leadership programs," an oversight assigned to the Human Resources Department (County of Sonoma, 2021b, p. 11).

The County of Sonoma also has a workforce development program that offers a variety of on-demand classes and training that supports employees in developing skills and competencies to become successful in their careers (County of Sonoma, 2021e). Sonoma County employees identified the following key competencies that the county needs to focus on: developing integrity and trust, customer service, passion \& energy, relationship management, innovation, systems thinking, etc. (County of Sonoma, n.d.).

\section{Groups that support employee development:}

Employee Resource Group (ERG): "are voluntary, employee-led groups that foster a diverse, inclusive workplace aligned with the organization's mission, values, goals, and business practices. ERGs often support the development of future leaders, increase employee engagement, and help expand employee involvement and outreach" (County of Sonoma, 2021g).

Sonoma County LatinX Employee Resource Network (SoCoLearn): a subsidiary of the ERG that supports "County departments, agencies, and programs as they improve their connection to the Latinx community" (County of Sonoma, 2021).In addition, they also support its members' "professional development, employee experiences, and help increase Latinx recruitment and retention" (County of Sonoma, 2021g).
BP 8: Accountability for the results of diversity programs is another attribute of best-practice organizations. Accountability is determined through metrics, surveys, focus groups, customer surveys, management, employee
One of the stated core values of the County of Sonoma is accountability. For Sonoma, accountability is expressed in "taking ownership of its actions and being fiscally responsible and results-driven, so that its success is aligned with community outcomes" (County of Sonoma, 2021b, p. 2). 
evaluations, and training and

education evaluations.

\section{UNIQUE DIVERSITY MANAGEMENT STRATEGIES}

\section{Strategies in Organizational} Readiness
- The county will "conduct an employee engagement survey by mid-2022, and based on survey data, develop and implement strategies to incorporate survey outcomes into future operational planning" (County of Sonoma, 2021b, p. 11).

- The county will "conduct a baseline assessment by mid-2022 of racial equity awareness and understanding among County staff and develop a process to assess progress annually" (County of Sonoma, 2021b, p. 12).

- The county will "establish a process for engagement and collaboration with community members and stakeholder groups, and launch a community engagement strategy by the end of 2022 with a focus on racial equity" (County of Sonoma, 2021b, p. 12).

\section{SPECIAL EMPLOYEE BENEFITS}

Employee Assistance Program (EAP)

\section{Other Benefits}

Employee Assistance Program (EAP) is available for both employees and their families to provide guidance and support in reaching their "professional goals, manage daily stresses, and develop fulfilling relationships" (County of Sonoma, 2021f) They offer counseling services, legal services, financial services, and work/life services (e.g., adult care, caregiver support, education services, information on parenting, autism family support services, etc.) (County of Sonoma, 2021f).

objectives that are poised to promote Sonoma County as an employer of choice with a diverse workforce is to "implement programs and identify opportunities to support employee work-life balance and a positive work environment, including a Telework Policy" (County of Sonoma, 2021b, p. 11). 


\section{Workforce Representation Analysis - Sonoma}

The following data analyzes the ethnicity of the County of Sonoma's 2021 workforce compared to the county's population and its available labor market workforce within its geographic region.

\section{Representation:}

- For 2021, White (68\%) employees have the highest representation among the four major ethnicities, followed by Hispanic or Latino $(21 \%)$ and then Asian (3\%), and followed closely by Black or African American (2\%).

- The employee representation that exceeded their countywide population was the White employee group exceeding by $5.1 \%$. All the other three major ethnicities, Hispanic or Latino group ($6.3 \%)$, Asian group (-1.6\%), and Black or African American group $(-0.10 \%)$, are underrepresented.

- For the Officials and Administrators job category, both African American and White employee groups are overrepresented. On the other hand, Hispanic and Asian employee groups are underrepresented, with Hispanics being the most under-represented in leadership $(-21.30 \%)$.

\section{Utilization:}

- The county's employee workgroups that are roughly equal to or exceed the available workforce are Black or African American, which exceeds by $0.6 \%$, and White, exceeding it by $3.27 \%$. On the other hand, Hispanic/Latino employees (-4.61\%) and Asian employees (-1.24\%) are underutilized.

- Regarding representation in leadership positions, particularly for the county's Officials and Administrators, the white (84\%) employee group has the highest representation among the four major ethnicities. It has also exceeded the region's available labor market supply of officials and administrators by $5 \%$. In addition, the Black or African American employee group has also exceeded their labor market supply in this category by $2.3 \%$.

- On the other hand, both Hispanic/Latino (-6.4\%) and Asian (-1.24\%) are the employee groups that are underutilized in the officials and administrator category within Sonoma County.

\section{COUNTY OF SONOMA WORKFORCE}

- County Population Estimate $\quad$ County Labor Market $\quad$ County Employees

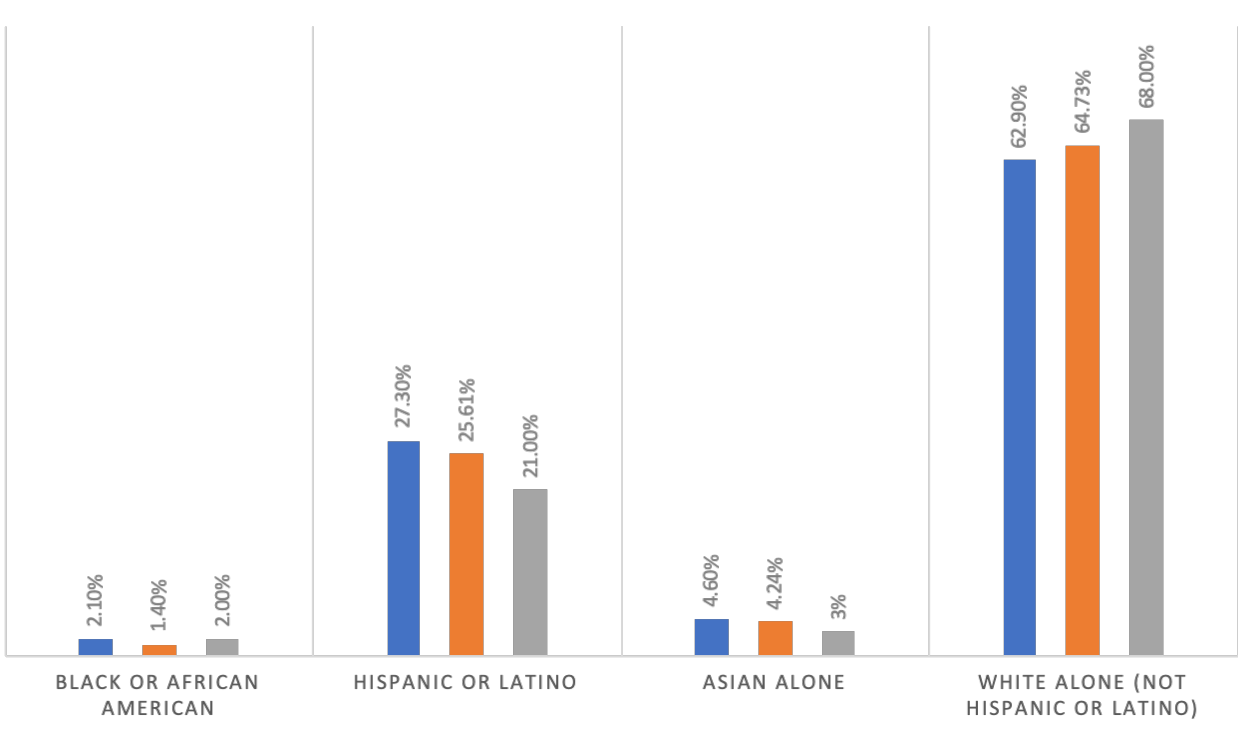

\section{COUNTY OF SONOMA OFFICIALS AND ADMINISTRATORS}

- County Population Estimate @ County Labor Market @ County Employees

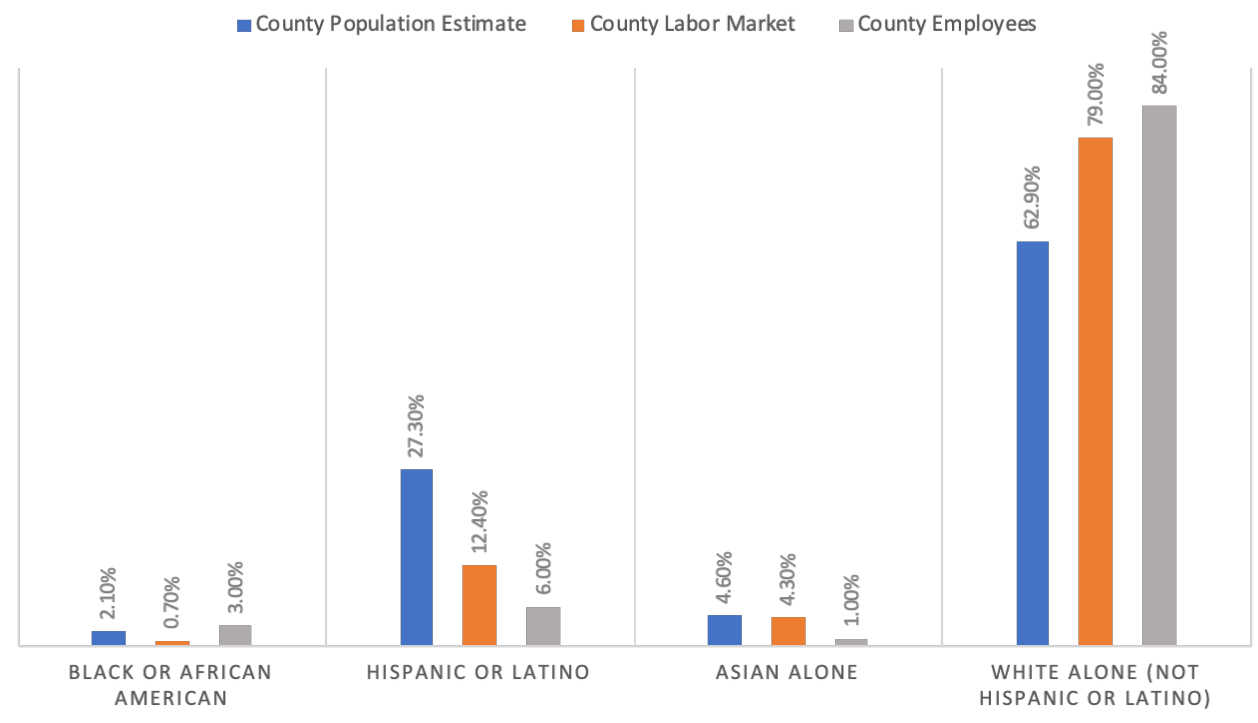




\section{Contra Costa County}

Contra Costa County is located in the northern part of the East Bay region of the San Francisco Bay Area. This region has an overall population size of approximately 1,153,526 (US Census Bureau, 2021). As of 2021, the county organization has a total workforce of 10,748 employees (A. Elliot, personal communication, April 9, 2021).

Contra Costa County's diversity management strategies are contained in its Equal Employment Opportunity Outreach and Recruitment Plan. It includes programs focusing on recruitment and outreach strategies to address underrepresentation within its workforce and its diversity training (Contra Costa, 2017).

Drivers of creating the plan: The Equal Employment Opportunity Outreach and Recruitment Plan express the Board's commitment to having a "diverse County workforce and to providing equal employment opportunities for all qualified persons seeking employment" and to “establish equal employment opportunity outreach and recruitment protocols designed to increase the diversity of the County workforce" (Contra Costa County, 2017).

A draft of a Racial Equity Action Plan is currently being finalized, and the Office of Racial Equity and Social Justice is now in its planning stage. Though not officially effectuated at this time, both of these plans do mention diversity management initiatives (Contra Costa County, 2020a). The stated mission of the Office of Racial Equity and Social Justice is:

To make a transformational shift within County government to eliminate inequity, harm, discrimination and bias (implicit and explicit) based on race, ethnicity, gender, sexual orientation, language, immigration status, socio-economic status, and for people with 
disabilities by focusing County efforts on understanding what it takes to achieve equity with a priority focus on racial equity. (Contra Costa County, 2020a)

The county has also participated in the Government Alliance on Race and Equity (GARE). Its cohort was responsible for developing the draft of its Racial Equity Action Plan (Contra Costa County, 2020a). 


\section{Diversity Management Programs - Contra Costa County}

BP 1: The development of a formal diversity process that is contained in laws, rules, or procedures. Both human and financial time and resources are devoted to the program. In best practice organizations, diversity is a process that is an integrated, ongoing, and measurable strategy.

BP 2: Diversity efforts are primarily decentralized, with a central governing body outlining the requirements of the plans, with individual agencies and departments developing their own plans that are tailored to their specific needs. This reinforces a sense of ownership and ensures that managing diversity has both top-level support and is a reality throughout the organization.
Although the county is currently in the process of establishing an Office of Racial Equity and Social justice, it still does not have a countywide program (Contra Costa Public Records Request, personal communication, April 9, 2021). Based on the proposal to the Board, the plan seeks $\$ 600,000$ annual revenue from the county's general fund to fund its operations (Contra Costa County, 2020a).

\section{BP 3: In best-practice} organizations, diversity training is provided to the workforce. The training is not limited to managers but is extended throughout the workforce. Successful organizations incorporate diversity into mentoring efforts, leadership
Undetermined. It is stated in the Office of Racial Equity proposal that the Board of Supervisors will determine the consolidation of existing county equity efforts from different departments after the completion of the Community Planning Process (Contra Costa County, 2020a).
The following is a list of diversity training for Contra Costa County employees.

\section{Required Training}

Workplace Diversity Training: The goals of this training are to promote awareness and encourage acceptance, describe strategies to create a positive and accepting work environment, and highlight the steps to take should discrimination occur in the workplace (Contra Costa Public Records Request, personal communication, April 9, 2021). Per the Boards' mandate, all employees are required to participate in this 1-hour training once every three years (Contra Costa Public Records Request, personal communication, April 9, 2021). 
training, and management-byresults programs.

\section{Optional Training}

Implicit Bias Awareness (IBA) Training: IBA is a three-hour virtual session "designed to expose attendees to their implicit biases, provide tools to adjust automatic patterns of thinking, and ultimately eliminate discriminatory behaviors" (Contra Costa Public Records Request, personal communication, April 9, 2021).

Implicit Bias for Supervisors and Managers (IBSM) Training: Currently, over a three-hour virtual delivery, this training assists supervisor and managers in recognizing their biases and tendencies that can influence the overall equity of hiring, performance evaluations, and promotions (Contra Costa Public Records Request, personal communication, April 9, 2021).

\section{Racial Equity Action Plan Proposed Training}

Contained in the copy of the Racial Equity Action Plan draft is implementing a "countywide mandatory introductory training on racial equity and immigrant inclusion, followed by ongoing training opportunities for all staff and elected officials. The training should include a variety of case studies and examples related to the jobs of those being trained. Training concepts should include the social construction of race, the history of race in government, implicit and explicit bias, institutional and structural racism, and tools and strategies to address bias and improve inclusion. Additional topicspecific training will include: using the Racial Equity Tool, inclusive outreach, and public engagement, and communicating about race" (Contra Costa County, 2020b, p. 13). The county will also identify standardized content and framework for its future training such as: "Cultural Intelligence, Structural Racism, Racial Equity Tool, Anti-Bias, How to participate in the hiring panel and process-based decision making” (Contra Costa County, 2020b, p. 14).

\section{BP 4: Best practice} organizations utilize workforce data and demographics to compare statistics reported for the civilian labor force. Occupations with underutilization are identified, and goals are established to reduce the underutilization. All of the best-practice organizations add creative and innovative recruitment strategies that get
Contra Costa County released an Administrative Bulletin back in 2017 to "establish equal employment opportunity outreach and recruitment protocols designed to increase the diversity of the County workforce" (Contra Costa County, 2017, p. 1). The following are the stated procedures of this plan:

\section{Creating the Strategic Plan:}

The County's Equal Employment Opportunity Officer ("EEO Officer") will create a strategic plan to expand outreach to underrepresented groups in order to attract the most qualified candidates for employment who reflect the diverse community it serves. On an annual basis, the County's EEO Officer will review the strategic plan and modify strategic initiatives as needed to reflect the Board of Supervisors' commitment to targeted outreach and recruitment efforts as reflected in the County's workforce statistics.

The EEO Officer is responsible for implementing the strategic plan. The EEO Officer will provide quarterly updates on the progress of the strategic plan to the Hiring Outreach Oversight Committee ("Committee"). At the 
results and set their programs apart. end of every calendar year, the EEO Officer will present an annual report to the Committee regarding the strategic plan. The annual report must include: (1) the details of the strategic plan; (2) the results of the EEO Officer's efforts to expand outreach to underrepresented groups; and (3) recommendations for specific outreach initiatives for the following year. Once the Committee has approved the specific outreach initiatives, the EEO Officer will report the updated Equal Employment Opportunity Outreach Plan to the entire Board of Supervisors for final approval.

The EEO Officer will provide the departments with tools and resources to conduct outreach and recruitment consistent with the countywide strategic outreach and the department's outreach and recruitment objectives (Contra Costa County, 2017, p. 1-2).

\section{Department Strategic Outreach:}

At the beginning of every calendar year, the EEO Officer will provide each department with the statistics that show the composition of that department's employees (broken down by Male, Female, White, Black, Hispanic and Asian) as compared with the available workforce in Contra Costa County. If a department has an underrepresented group of employees and the degree of underrepresentation is at least $10 \%$, the department shall develop an Action Plan for targeted outreach and recruitment to that group. The department will be expected to present its Action Plan to the Hiring Outreach Oversight Committee at the end of the fiscal year. The department may work independently or in conjunction with the EEO Officer to determine an effective Action Plan to address the ethnic or gender groups that are underrepresented. The EEO Officer will provide the department with tools and resources to create an Action Plan.

An Action Plan may include any of the following:

- developing recruitment plans to reach the underrepresented populations

- developing and implementing outreach plans

- participating in job and diversity fairs

- coordinating with schools and professional organizations

- offering internships

- ensuring oral boards and screening committees reflect the diversity of the applicant pool

The department will return to the Committee before the end of the next fiscal year to present the results of its efforts to expand outreach to underrepresented groups during the previous fiscal year.

Adverse Impact Review on Job Exam: A guide used by Human Resources to analyze and determine if an examination adversely impacts a particular group. The guide also gives tips and strategies for developing quality written examinations (Contra Costa Public Records Request, personal communication, April 9, 2021). 
BP 5: Best practice agencies have found that requiring diversity programs through the law, executive order, or other mandates compels agencies to establish serious goals and to make earnest efforts toward meeting those goals.

\section{BP 6: Best practice}

organizations have established a review committee responsible for establishing policies, providing technical assistance, reviewing/approving plans, and monitoring progress toward achieving goals.
The County Administrator has the authority and the responsibility to establish and enforce the procedures and requirements included in the Equal Employment Opportunity Outreach and Recruitment Plan (Contra Costa County, 2017). Such procedures require departments to produce action plans if underutilization is reported and present reports to the Hiring Outreach Oversight Committee on their progress (Contra Costa County, 2017).
BP 7: Effective diversity programs also link recruitment, development, and retention strategies to organizational performance. They integrate employee development processes and map career paths to see what critical skills are necessary to advance, then communicate these skills to employees and provide training.
"The Hiring Outreach Oversight Committee was established by the Board of Supervisors to review the statistical data of minorities and females in the County's workforce and make recommendations to target outreach and recruitment in those communities where the County workforce is not reflective of the diversity of the County. The committee is scheduled to meet four times per year" (Contra Costa County, 2021a). This Committee is responsible for monitoring, reviewing and making recommendations on recruitment and outreach action plans.
BP 8: Accountability for the results of diversity programs is another attribute of best-practice organizations. Accountability is determined through metrics, surveys, focus groups, customer
This research was not able to find any program that aligns with BP 7.

Mentioned in the Racial Equity Action Plan draft is the proposed development of a Racial Equity Tool that is meant to assess the impact of personnel policy and practices (Contra Costa County, 2020b, p. 13).

Also mentioned in the draft is the use of the Outreach and Recruitment Report, provided by the county's Equal 
surveys, management, employee evaluations, and training and education evaluations.
Employment Officer, to show Human Resources and various departments data on underrepresentation in gender and race (Contra Costa County, 2020b, p. 13).

\section{SPECIAL EMPLOYEE BENEFITS}

Employee Assistance Program Contra Costa County's EAP services provide employees and their families 24/7 confidential telephonic consultation and (EAP) referrals on parenting, professional growth, aging, healthy living, work-life balance, communication, stress and anxiety, and legal and financial coaching (Contra Costa County, 2021b). They also provide resources such as child and elder care assistance or health and wellness (Contra Costa County, 2021b).

\section{Other Benefits}

Kids at Work Child Care Center: The county offers its employees an affordable onsite preschool and infant care service at their Public Works facility. This non-profit infant center provides care for children eight weeks old to kindergarten, which comes with meals and experienced staff (Contra Costa County, 2021c).

Dependent Care Assistance Program (DCAP): This benefit allows county employees to set aside pretax dollars to pay for eligible dependent expenses such as child care (Contra Costa County, 2021d).

Catastrophic Leave: This is designed to assist eligible county employees who have exhausted all paid accruals due to a serious or catastrophic illness, injury, or condition of the employee or family member. The program establishes and maintains a county-wide bank wherein any employee who wishes to contribute may authorize that a portion of his/her accrued vacation, compensatory time, holiday compensatory time or personal holiday credit be deducted from those account(s) and credited to the Catastrophic Leave Bank. Employees may donate hours to a specific eligible employee or the general bank (Contra Costa County, 2021e).

Commuter Benefit Program: tax advantage set aside money that employees can use to pay for transportation. A debit card will be issued to the employee that they can use for transportation related expenses (Contra Costa, 2021f). 


\section{Workforce Representation Analysis - Contra Costa}

The following data is an analysis of Contra Costa County's 2021 workforce's ethnicity, compared to its population and its available labor market within its geographic region.

Representation:

- For 2021, White (37\%) employees have the highest representation among the four major ethnicities, followed by Hispanic or Latino $(19.28 \%)$, then Black or African American (14.50\%), and followed by Asian (12.78\%)

- Black or African American is the only employee group that exceeded their representation (5\%) compared to its overall population. All the other three major ethnicities, Hispanic or Latino group (-6.72\%), Asian group (-5.52\%), and White group (-5.7\%), are underrepresented.

- For the Officials and Administrators job category, both African American and White employee groups are overrepresented. On the other hand, Hispanic and Asian employee groups are underrepresented, with Hispanics being the least represented ($14.42 \%)$.

Utilization:

- Similar to the population representation, Black or African American is the only employee group among the four that exceeded its representation in the available workforce. On the other hand, Hispanic/Latino (-5.2\%), Asian (-3.9\%), and white $(-8.55 \%)$ are each underutilized.

- Regarding representation in leadership positions, particularly for the county's Officials and Administrators, the White (55\%) employee group has the highest representation among the four major ethnicities. Still, it is underutilized compared to its available labor market supply of officials and administrators within the region (4.6\%). The Black or African American employee group has exceeded their labor market supply in this category by $8.24 \%$.

- On the other hand, both Hispanic/Latino (-1.22\%) and Asian (-9\%) are the employee groups that are underutilized in the officials and administrator category within Contra Costa.

\section{CONTRA COSTA COUNTY WORKFORCE}

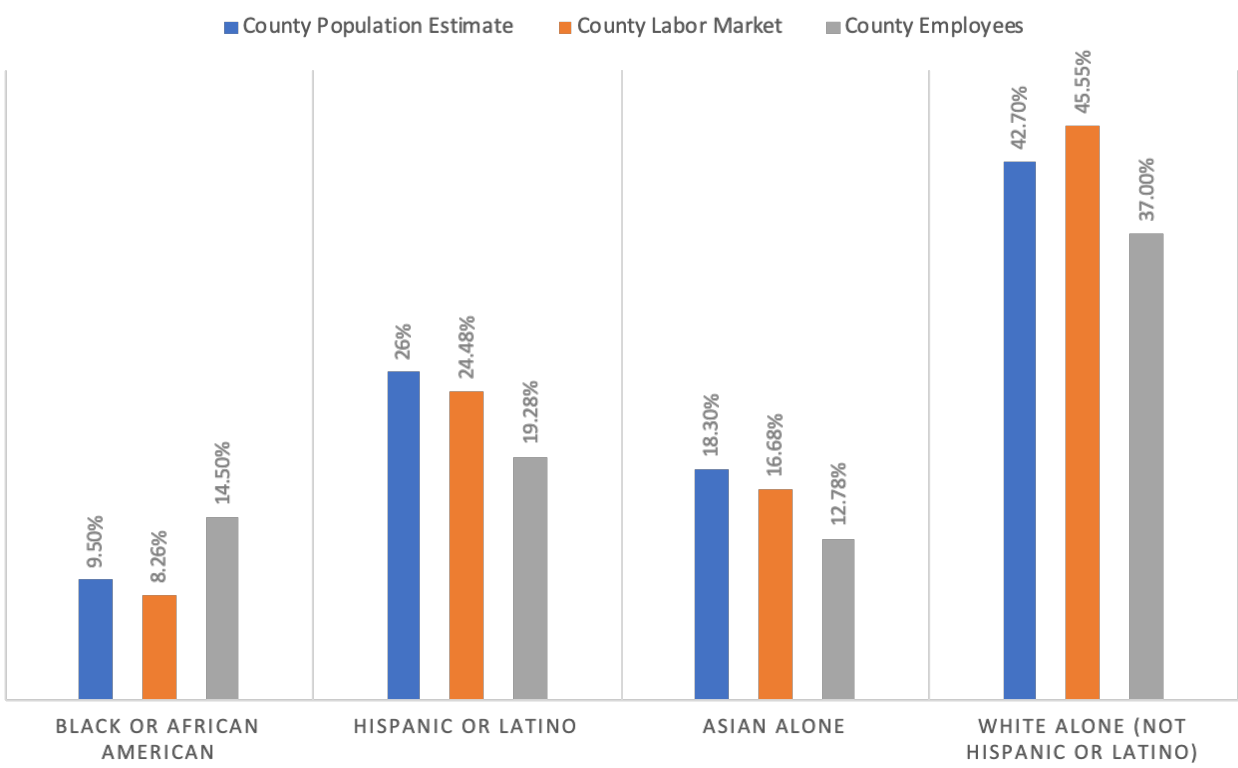

\section{CONTRA COSTA COUNTY OFFICIALS AND ADMINISTRATORS}

- County Population Estimate = County Labor Market =County Employees

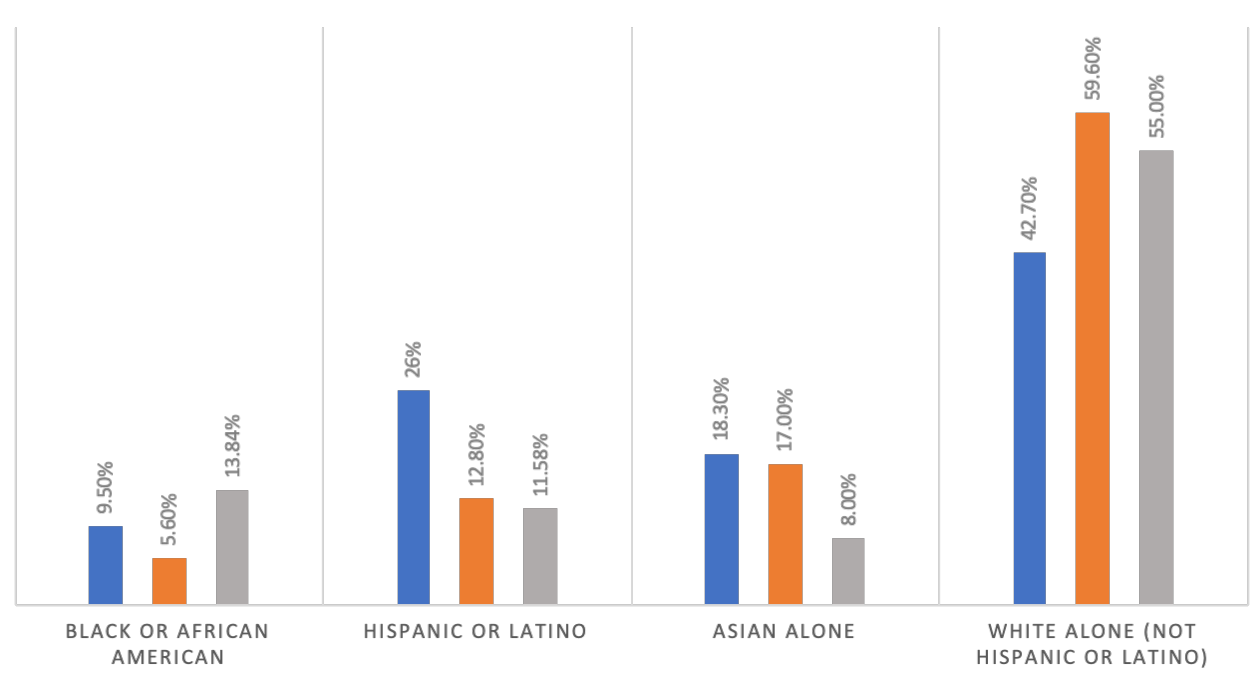




\section{County of Santa Clara}

The County of Santa Clara is located on the southern coast of San Francisco Bay. It is home to Silicon Valley, where a host of high-tech companies and technology giants such as Facebook, Google and Apple can be found. This region has an overall population size of approximately 1.92 million (US Census Bureau, 2021). Santa Clara is the most populous jurisdiction in the nine-county Bay Area Region. As of November 2020, the county organization has a total workforce of 17,185 employees (County of Santa Clara, 2020).

Since the early 1990s, the County of Santa Clara has recognized the value of workforce diversity as stated in its Board of Supervisors' Policy on Diversity (County of Santa Clara, 1993). The policy states:

Santa Clara County's workforce has, for some time, reflected the "quilt" that is our community. We recognize that diversity includes more than cultural groups and that valuing diversity requires a change in the way we do business and how we interact with each other. Valuing diversity requires a change in our organizational culture.

We believe that a diverse workforce provides advantages both internally, in terms of the human resources potential offered by a variety of diverse perspectives, and externally, in increasing the county's ability to respond to an equally diverse community. In order to treat people fairly and provide equal opportunity, it is not enough to treat all people the same. The organization must instead respect all people and seek to accommodate and learn from the different perspectives and values they contribute. (County of Santa Clara, 1993) 
In 2017 this policy was updated to expand its recruitment and outreach strategies, including removing its statement on affirmative action (County of Santa Clara, 2017). In this revised policy, the following objectives have been mentioned as the county's key focus in promoting workforce diversity: action plans about outreach and recruitment efforts, ensuring equal opportunity and upward mobility of the diverse workforce, and promoting an organizational culture that appreciates diversity and that is recognized on every level of the organization (County of Santa Clara, 2017). Additionally, the plan also seeks to "conduct employee training to help create an openness to the experience of others" and will "hold all managers accountable for demonstrating leadership in valuing diversity" (County of Santa Clara, 2017, p. 2). This research was not able to locate specific action plans that are tied to each of these goals.

More recently, the county has sent seven members from its workforce to participate in the Government Alliance on Racial Equity (GARE) to assist in the assessment and development of countywide racial equity initiatives (M. Love, personal communication, January 15, 2021). Additionally, the county has also created its first Division of Equity and Social Justice (DESJ) under the County Executive's Office. It has appointed its first Deputy County Executive to serve as the Chief Equity Officer for this Division (County of Santa Clara, 2021). Dr. Rocio Luna, Deputy County Executive for DESJ, hopes to review the best practices in diversity management to support the county's diversity goals (R. Luna, personal communication, April 19, 2021).

Drivers of creating the plan: The recent reignited attention given to racial equity was driven by reports that were received by the Board of Supervisors on the GARE efforts and have provided further direction to advance it. In addition, the recent events in society (e.g., the Black Lives Matter movement and associated awareness of the structural racism facing people of 
color), as well as the principle that the county workforce should reflect the community that it serves, are some of the contributors to these recent initiatives (J. Mills, personal communication, February 2, 2021). There is also a growing expectation from employees and community members to create a solid approach to diversity goals at all levels of the organization (R. Luna, personal communication, April 19, 2021). 


\section{Diversity Management Programs - County of Santa Clara}

BP 1: The development of a formal diversity process that is contained in laws, rules, or procedures. Both human and financial time and resources are devoted to the program. In best practice organizations, diversity is a process that is an integrated, ongoing, and measurable strategy.
Even though the Board of Supervisors Policy on Diversity exists, there is no formal active and consistent implementation team/approach to ensure this policy actively meets its intended goal. It is currently a passive policy, versus an active policy, that is tracked and reported on (R. Luna, personal communication, April 19, 2021).
BP 2: Diversity efforts are primarily decentralized, with a central governing body outlining the requirements of the plans, with individual agencies and departments developing their own plans that are tailored to their specific needs. This reinforces a sense of ownership and ensures that managing diversity has both top-level support and is a reality throughout the organization.
Though this is not implemented across the entire organization, the county's Public Health Department has its own racial and health equity strategic initiative to help eliminate healthcare provision disparities. It is also mentioned that the Public Health Department is "convening a workforce equity team to create a workplace culture where racial equity is valued and operationalized in order to establish a pipeline program to diversify the workforce" (Santa Clara County Public Health, 2021).
BP 3: In best-practice organizations, diversity training is provided to the workforce. The training is not limited to managers but is extended throughout the workforce. Successful organizations incorporate diversity into mentoring efforts, leadership
Though not required, the Learning and Employee Development Department provides diversity training classes that are available on-demand (R. Luna, personal communication, April 19, 2021). Diversity training is one of the several pieces of training mentioned on the Equal Opportunity Division website, including the sexual harassment and discrimination training for both supervisors and non-supervisory and reasonable accommodation (County of Santa Clara Equal Opportunity Division, 2021). "This workshop is a facilitated process in which participants explore what divides us in our society and the process of conditioning that we all go through and how that affects us in dealing with each other" (County of Santa Clara Equal Opportunity Division, 2021). 
training, and management-byresults programs.

\section{BP 4: Best practice} organizations utilize workforce data and demographics to compare statistics reported for the civilian labor force.

Occupations with underutilization are identified, and goals are established to reduce the underutilization. All of the best-practice organizations add creative and innovative recruitment strategies that get results and set their programs apart.

\section{$\underline{\text { Workforce Data Collection }}$}

The county's Employee Services Agency (ESA) data team now has access to workforce data, which has been used to examine representation in each of the occupations at the county. Efforts to create a robust strategic plan to address utilization gaps have been underway for quite some time (J. Mills, personal communication, February 2, 2021).

\section{$\underline{\text { Recruitment and Outreach Strategies }}$}

The following are recruitment and outreach strategies mentioned in the Board of Supervisor Policy on Diversity, consistent with Government Code section 11139.6:

- "Engage in general recruitment and outreach programs to all individuals, including persons who are economically disadvantaged" (County of Santa Clara, 2017).

- "Engage in inclusive public sector outreach and recruitment programs that, as a component of general recruitment, may include, but not be limited to, focused outreach and recruitment of minority groups (defined by race, ethnicity, and/or sexual orientation) and women if any such group is underrepresented in entry level positions at the County or determined to be significantly underutilized at any level of position at the County. Such focused outreach and recruitment may include, but is not limited to, placement of job announcements through" (County of Santa Clara, 2017, p. 2):

$\circ$ General circulation newspapers, general circulation publications, and general market radio and television stations, including electronic media.

- Local and regional community newspapers.

- Newspapers, publications, and radio and television stations that provide information in languages other than English and whose primary audience is residents of minority and low-income communities.

- Publications, including electronic media, that are distributed to the general market and to newspapers, publications, and radio and television stations whose primary audience is comprised of minority groups or women.

- Recruitment booths at job fairs or conferences oriented to both the general market and the economically disadvantaged as well as those events drawing a significant participation by minorities or women.

Additional recruitment and outreach strategies mentioned in EEO Utilization report that are meant to address the reported underutilization include (County of Santa Clara, 2019a, p. 5):

- The County will continue to post all job announcements (open-competitive, promotional, and transfers) on the County's website. The County will continue to accept applications through its online application system. 
- The County will continue to offer kiosks at three locations in County buildings for those applicants without access to a computer. Additionally, computers are available at various public libraries, including County libraries, for public use for filing online applications with the County.

- The County will continue to update job specifications to ensure all qualified applicants are encouraged to apply.

- To ensure access throughout the application process, the County will continue to provide subject matter test qualifications during nights and weekends for flexibility.

- The County will continue to conduct oral examinations with a diverse panel of evaluators.

- The County will continue to offer Skype for out-of-the-area candidates for oral examination and hiring interviews.

It was also noted that the ESA has a service agreement to post job bulletins to diverse bulletin boards, but targeting could be more strategic, given additional resources and focus (J. Mills, personal communication, February 2, 2021).

BP 5: Best practice agencies have found that requiring diversity programs through the law, executive order, or other mandates compels agencies to establish serious goals and to make earnest efforts toward meeting those goals.
County agencies and departments are not required to comply with any diversity management initiative. No serious efforts to meet diversity management goals have been established as a universal practice (J. Mills, personal communication, February 2, 2021).

\section{BP 6: Best practice}

organizations have established a review committee responsible for establishing policies, providing technical assistance, reviewing/approving plans, and monitoring progress toward achieving goals.
The Public Health Department is said to be convening its own workforce equity team; however, this research could not find any indication that this involves a countywide oversight. It was also mentioned that a Race/Equity Steering Committee is in the works. This committee is meant to oversee service delivery and the workforce (J.Mills, personal communication, February 2, 2021).
BP 7: Effective diversity programs also link recruitment, development, and retention strategies to organizational
The county currently has no diversity action plan or strategies in place that actively promote employee development. This will be a structural challenge for the county at the moment since employee learning and development (LED) and recruitment/retention are overseen by two separate departments (CEO's Office and ESA) (J. Mills, personal communication, February 2, 2021). 
performance. They integrate employee development processes and map career paths to see what critical skills are necessary to advance, then communicate these skills to employees and provide training.

BP 8: Accountability for the results of diversity programs is another attribute of best-practice organizations. Accountability is determined through metrics, surveys, focus groups, customer surveys, management, employee evaluations, and training and education evaluations.
The county has a wealth of on-demand resources and training from its SCCLearn portal (J. Mills, personal communication, February 2, 2021). The county also has a robust tuition reimbursement program available to all full-time county employees to encourage employee development (D. Perez, personal communication, February 2, 2021).

\section{UNIQUE DIVERSITY MANAGEMENT STRATEGIES}

Initiatives Promoting Inclusion Santa Clara County is the first county in California to observe Juneteenth as a paid holiday (J. Mills, personal communication, February 2, 2021).

Initiatives Promoting Equity Pay Equity Dashboard: As one of the many ways to show its commitment and accountability in ensuring equal employment opportunity across its organization, the County of Santa Clara has created a publicly accessible pay equity dashboard with the help of the Employee Services Agency Data Team. This is a workgroup collaboration comprised of members of the Employee Services Agency (ESA), County Counsel, the Equal Opportunity Department (EOD), and the Office of Women's Policy (OWP), convened to provide information and analysis relating to how the county is doing as an employer relative to pay equity (County of Santa Clara, 2021). 
Employee Assistance Program The county's EAP program offers the following services (County of Santa Clara, 2021):

(EAP)

- Counseling: Up to five free, confidential, easy-to-schedule appointments.

- Consultation: Consultation and coaching to managers, supervisors and union stewards on sensitive employee issues including how to approach a troubled employee in need of help.

- Assessment \& Referral: Referral as needed to a range of community resources such as parenting classes, self-help groups, 12-steps groups, elder care services and others.

- Training: Specialized training as requested by the department (e.g., Stress Management, Conflict and Communication, Balancing Work and Home).

- Classes: Coping with Grief and Loss, The Three Stages of ParentCare, Retirement: Ready or Not?, Write On! Writing for Fun and Health, Addiction: Why Can't it Just Stop?, Lifescripts

- Critical Incident Debriefings: Provide scheduled EAP response to critical incidents.

- Lending Library: Hundreds of self-help books available for EAP clients.

Other Benefits

Dependent Care Assistance Program (DCAP): A tax-advantage employee assistance program that allows employees to set up a special account for paying dependent care services such as the care for dependent children under age 13 and the care for another dependent who is physically or mentally incapable of caring for him/herself. This includes elder daycare (County of Santa Clara Employee Services, 2021a).

VTA Smart Pass: "The SmartPass Clipper Card can be used for unlimited rides on regular service for the VTA bus or light rail systems" (County of Santa Clara Employee Services, 2021b).

Vacation Donation: Employees can donate their vacation hours to those who need additional hours for qualifying requests or situations (D. Perez, personal communication, August 8, 2021). 


\section{Workforce Representation Analysis - Santa Clara}

The following data analyzes the ethnicity of the County of Santa Clara's 2021 workforce compared to the county's population and its available labor market within its geographic region.

\section{Representation:}

- For 2021, Asian (32\%) employees have the highest representation among the four major ethnicities, followed closely by Hispanic or Latino $(26.70 \%)$ and then white $(19.50 \%)$, and finally by Black or African American (5.20\%).

- The employee representation that exceeded their countywide population is the Black or African American group exceeding by $2.4 \%$ and the Hispanic or Latino group exceeding by $1.7 \%$. Both the Asian group (-7\%) and the White group (-11\%) are underrepresented.

- For the Officials and Administrators job category, African American is the only employee group that is overrepresented. Hispanic, White, and Asian employee groups are all underrepresented, with Hispanics being the least represented in leadership $(-21.30 \%)$.

\section{Utilization:}

- Mirroring the outcome on the population representation, the Black or African American employee group and Hispanic/Latino group exceed their available countywide workforce by $2.72 \%$ and $1.98 \%$, respectively. And again, both Asians (-4.41\%) and White (-13.5\%) are underutilized compared to their available countywide labor market.

- Regarding representation in leadership positions, specifically for the County's Officials and Administrators, the Asian (37.97\%) employees have the highest representation among the four major ethnicities. They have also exceeded the region's available labor market supply of officials and administrators by $2.07 \%$. In addition to the Asian employee group, the Black or African American employee group has also exceeded their labor market supply in this category by $2.72 \%$.

- On the other hand, both Hispanic/Latino (-7.22\%) and White ($25.04 \%$ ) are the employee groups that are underutilized in the officials and administrator category within Santa Clara County.

\section{COUNTY OF SANTA CLARA WORKFORCE}

- County Population Estimate $\quad$ County Labor Market County Employees

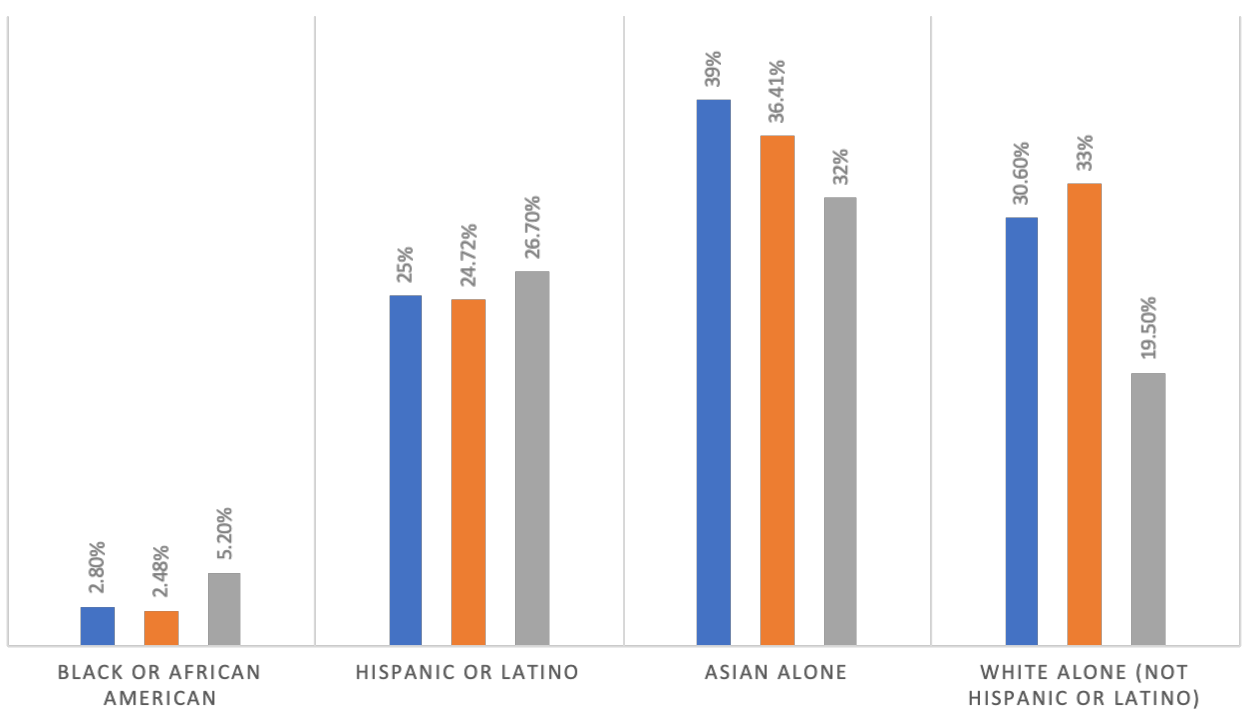

\section{COUNTY OF SANTA CLARA OFFICIALS AND ADMINISTRATORS}

- County Population Estimate = County Labor Market =County Employees

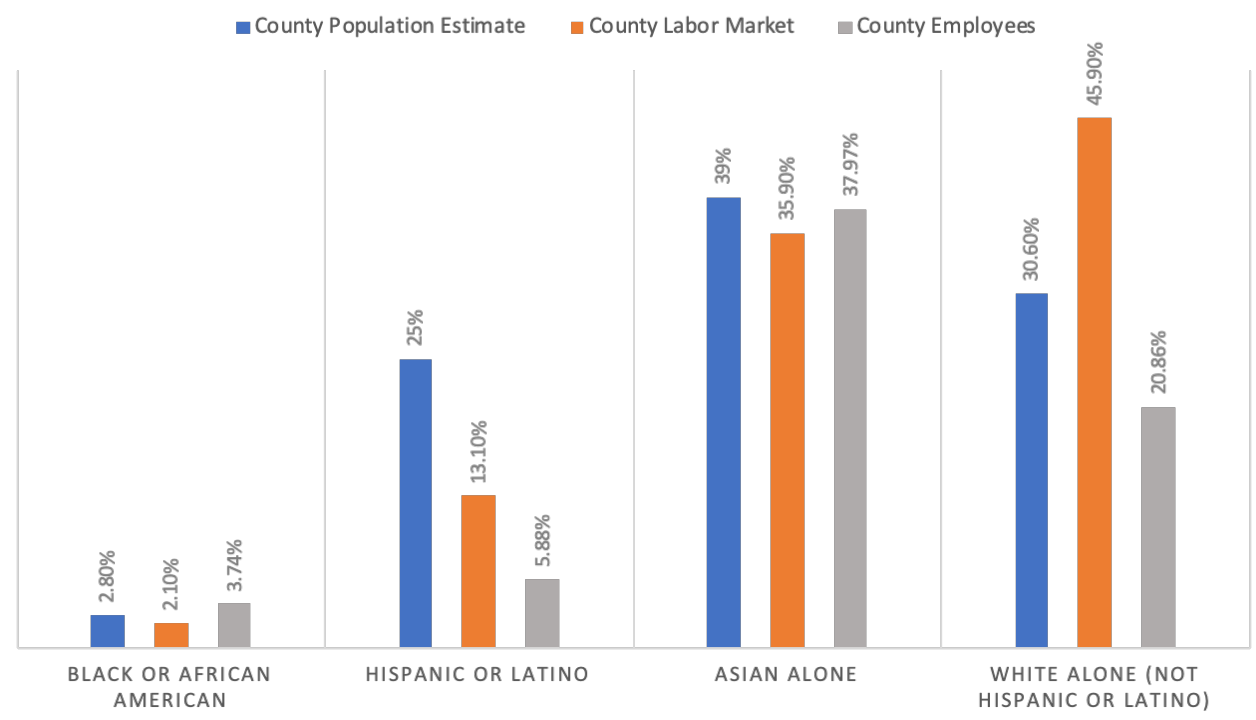




\section{Alameda County}

Alameda County, a region located on the east side of San Francisco Bay, has an overall population size of approximately 1,671,329 (US Census Bureau, 2021). As of 2019, the county organization had a total workforce of 8,296 employees (J. Angelo, personal communication, April 9, 2021).

Alameda County's Diversity Program belongs within the Office of the County Counsel. Its stated mission is:

To contribute to the goals of Alameda County, help drive business results and encourage employee engagement by working collaboratively with each county agency and department: to effectively manage and value the diversity of its employees; to promote a harassment-free workplace; to ensure inclusion of all people; to minimize the human and financial costs associated with protracted and adversarial processes; and, to help provide human-centered services. (Alameda County, 2021a)

The county's diversity program ensures that "employment policies and practices are nondiscriminatory," and it intends to help the County of Alameda "realize diversity and inclusion as an integral organizational strategy." The program's goal is to create a diverse workforce that respects and values cultural differences (Alameda County, 2021b).

One of the programs overseen by this unit is the Diversity \& Inclusion (D\&I) program.

The aim of this program is to integrate Alameda County's workforce with its policies, practices and systems in such a way as to maximize each employee's potential, with the opportunity to contribute to the County's mission of reflecting the population of the 
communities it serves in all its dimensions. As a whole the County seeks to have a culturally Competent workforce which is able to solve problems, make decisions and resolve conflicts in ways that optimize cultural differences for better, longer lasting, and more creative solutions. (Alameda County, 2021b)

This research could not locate or secure supporting Board ordinances or additional information about this program. 


\section{Diversity Management Programs - Alameda County}

\section{This research could not find any programs that align with BP 1 to BP 6, and BP 8.}

BP 7: Effective diversity programs also link recruitment, development, and retention strategies to organizational performance. They integrate employee development processes and map career paths to see what critical skills are necessary to advance, then communicate these skills to employees and provide training.
Career Ladders: a tool developed by Alameda County's Human Resource Services (HRS) to assist employees in navigating its job classification system and help them plan their career growth and trajectory. HRS has developed 90 "career ladders" or career paths specific from different Agencies and Departments. "Career ladders identify a sequence of job classifications through which an employee can typically progress from entry-level to positions of higher levels of pay, skill, responsibility, and authority" (Alameda County, 2021c).

Alameda County Training \& Education Center is a branch of Human Resource Services that provides innovative learning and development opportunities to promote individual and organizational excellence. This service is open to both its employees and the public. Some of the services they provide include quality classes; workshops and programs aimed at today's real business issues; in-depth training and development for managers, supervisors, and employees; and team and organizational development (Alameda County Training center, n.d.).

\section{SPECIAL EMPLOYEE BENEFITS}

Employee Assistance Program (EAP)
The county's EAP program is outsourced to Claremont Behavioral Services. Employees and their family members who need assistance will be confidentially recommended to counselors or resources that fit their needs. Some of the services include counseling, legal consultation, financial service, elder/adult/child care resources, school/college assistance for parents who need help in deciding to which college to send their children to, access to Clairmont's Personal Advantage website for online resources (Alameda County, 2021d).

\section{Other Benefits}

County Allowance: Alameda County offers employees an annual allowance to offset the cost of medical, dental, vision, supplemental employee life, and AD\&D insurance benefits elections (Alameda County, 2021e).

Dependent Care Assistance Program (DCAP): a tax-advantage program to assist employees on dependent care expenses (Alameda County, 2021e).

Alameda County Early Learning Center (Kidango): Alameda County's on-site child care center provided for its employees."Kidango has over 30 years of experience in early education through a wide variety of programs that meet the diverse needs of children and their families. The Center provides nutritious meals, a well-designed program for children three to five years, and a caring environment for families. The children are given the opportunity to learn and grow 
through specifically designed learning areas and age-appropriate activities and curriculum" (Alameda County, 2021f).

Commuter Benefits Program: "The Commuter Benefit Plan is where you can set aside before-tax dollars to pay for your mass transit and parking expenses as it relates to commuting to and from work. This could result in significant savings in the cost of your transportation and parking expenses through the taxes you save" (Alameda County, 2021g).

Catastrophic Sick Leave Program: “An employee may be eligible to receive donations of paid leave to be included in the employee's sick leave balance if $\mathrm{s} /$ he has suffered a catastrophic illness or injury which prevents the employee from being able to work or from being able to work his/her regularly scheduled number of hours" (Alameda County, $2021 \mathrm{~h}$ ). 


\section{Workforce Representation Analysis - Alameda County}

The following data analyzes the ethnicity of Alameda County's 2019 employee workforce compared to the county's population and the current workforce that is available countywide.

\section{$\underline{\text { Representation }}$}

- $\quad$ For 2019 , White $(30 \%)$ employees have the highest representation among the four major ethnicities, followed closely by Black or African American (25\%) and then Asian (23\%), and finally by Hispanic or Latino (17\%).

- Black or African American ( $+14 \%)$ is the only employee group that has exceeded their employee representation by a significant number compared to its countywide population. The Hispanic or Latino group (-5.3\%), Asian group (-9.3\%), and White (-.60\%) are all underrepresented.

- For the Officials and Administrators job category, both African American and White employee groups are overrepresented, with Black being the highest ( $+15.84 \%)$. Hispanic and Asian employee groups are underrepresented, with Asian employee groups being the least represented $(-18.21 \%)$.

\section{$\underline{\text { Utilization }}$}

- In terms of employee group representation exceeding its available workforce within the region, the Black or African American employee group once again is the only group that met this criterion. The Hispanic/Latino employee group (-4.9\%), Asian employee group (-6.65\%), and White $(-4.5 \%)$ are underrepresented when compared to its available countywide labor market.

- In terms of representation in leadership positions, particularly for the county's Officials and Administrators, the white (38.25\%) employee group has the highest representation among the four major ethnicities. Still, it is underrepresented compared to its available labor market supply of officials and administrators within the county.

- Both Hispanic or Latino (+1.92\%) and Black or African American $(+18.64 \%)$ employee groups have exceeded their labor market supply for officials and administrators. The Asian (-15.21\%) employee group is the only non-white employee group underrepresented in this category.

\section{ALAMEDA COUNTY WORKFORCE}

- County Population Estimate $\quad$ County Labor Market $\quad$ County Employees

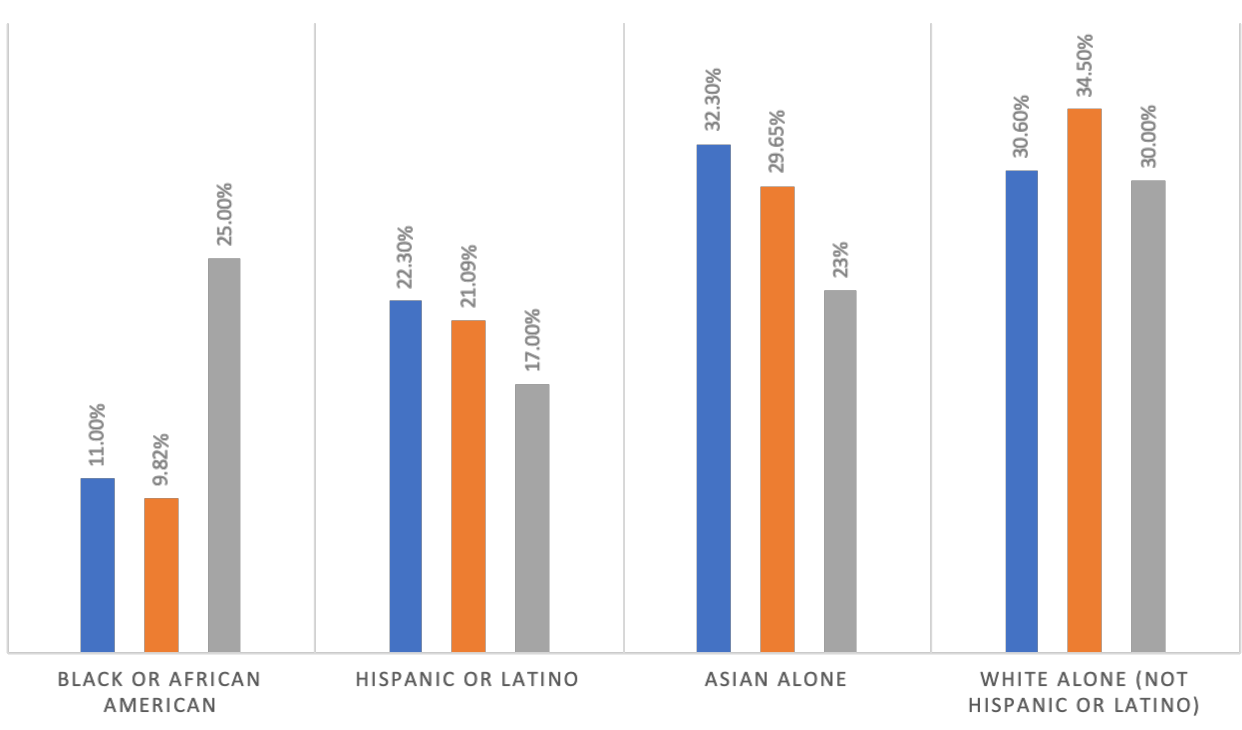

\section{ALAMEDA COUNTY OFFICIALS AND ADMINISTRATORS}

- County Population Estimate = County Labor Market =County Employees

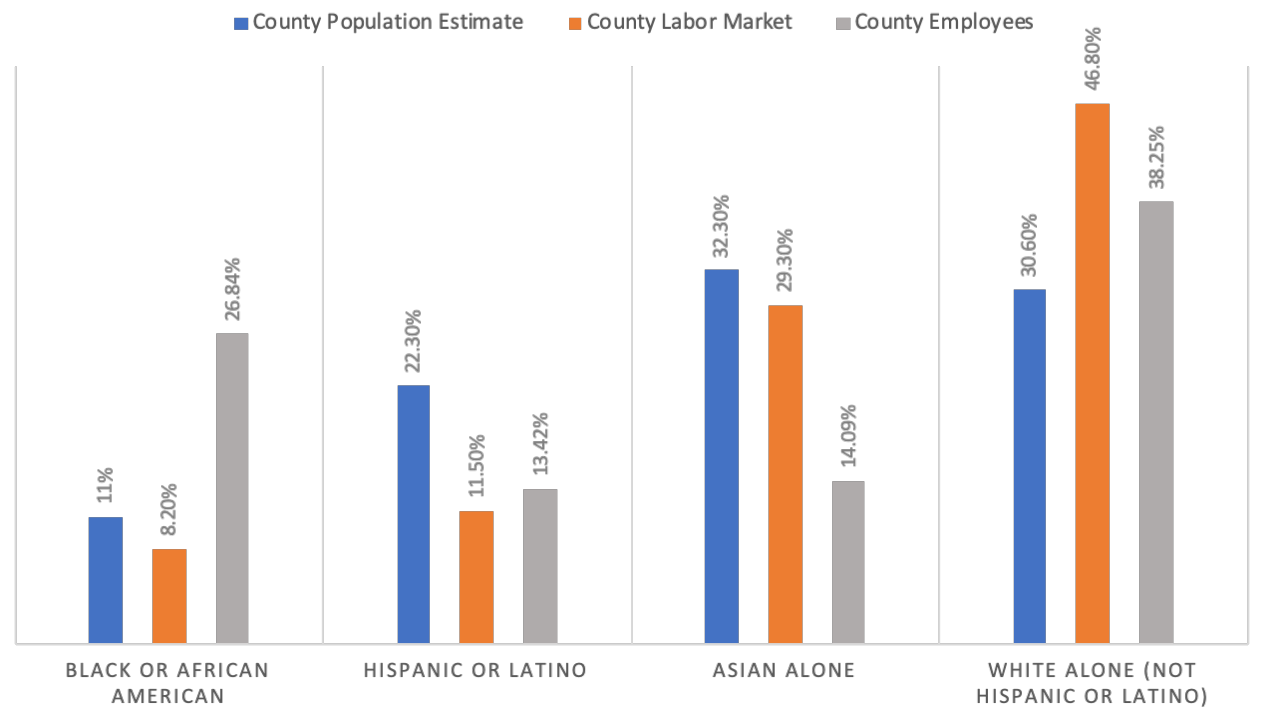




\section{Napa County}

Napa County, a region in Northern California renowned for its wines, is located in the northcentral part of the Bay Area region. This region has an overall population size of approximately 137,744 (US Census Bureau, 2021). As of 2021, the county organization has a total workforce of 1,366 employees (Napa Public Records Request, personal communication, April 20, 2021).

Napa County currently does not have a published diversity management program. Napa County, however, has a Diversity, Equity, Inclusion, and Belonging (DEIB) Committee, which is composed of the County Executive Officer, appointed and elected department heads, representation from the Board of Supervisors, and the Training and Organizational Development Officer (Napa Public Records Request, personal communication, April 20, 2021). The DEIB meets regularly to review and address issues related to diversity, equity, inclusion, and belonging in Napa County (Napa Public Records Request, personal communication, April 20, 2021). 


\section{Diversity Management Programs - Napa County}

This research only found one program that aligns with IPMA's best practices (BP 4).

BP 4: Best practice

organizations utilize workforce

data and demographics to

compare statistics reported for

the civilian labor force.

Occupations with under-

utilization are identified, and

goals are established to reduce

the underutilization. All of the

best-practice organizations add

creative and innovative

recruitment strategies that get

results and set their programs

apart.

\section{SPECIAL EMPLOYEE BENEFITS}

Employee Assistance Program (EAP)
Over the past two years, committee projects have included analyzing workforce demographic information, implementing "blind" screening of applications (removing identifying candidate information), and formalizing the county's use of gender-neutral language (personal communication, April 20, 2021) 


\section{Workforce Representation Analysis - Napa County}

The following data analyzes the ethnicity of Napa County's 2021 workforce compared to the county's population and the labor market available countywide.

Representation:

- For 2021, White (55.56\%) employees have the highest representation among the four major ethnicities, followed by Hispanic or Latino (29.36\%) and then Asian (6.66\%), and finally by Black or African American (4.83\%).

- The White $(+3.76 \%)$ and Black/African American $(+2.33 \%)$ employee groups have exceeded their employee representation compared to Napa's countywide population. The Hispanic or Latino group (-5.24\%) and Asian group (-2.24\%) are underrepresented.

- For the Officials and Administrators job category, both African American and White employee groups are overrepresented. In contrast, Hispanic and Asian employee groups are underrepresented.

Utilization:

- The county's employee workgroups that exceed Napa's available labor market are the following employee groups: Black or African American $(+2.93 \%)$ and White $(+2.57 \%)$. On the other hand,

Hispanic/Latino employees (-4.64\%) and Asian employees (-1.53\%) are underrepresented compared to their available countywide workforce.

- In terms of representation in leadership positions, particularly for the county's Officials and Administrators, the white (72\%) employee group has the highest representation among the four major ethnicities. It has also matched its available labor market supply of officials and administrators within the region. The Black or African American employee group has exceeded their labor market supply in this category by $5.2 \%$.

- On the other hand, both Hispanic or Latino (-4\%) and Asian (-1.7\%) employee groups are underutilized in the officials and administrator category within Napa County.

\section{NAPA COUNTY WORKFORCE}

- County Population Estimate - County Labor Market $\quad$ County Employees

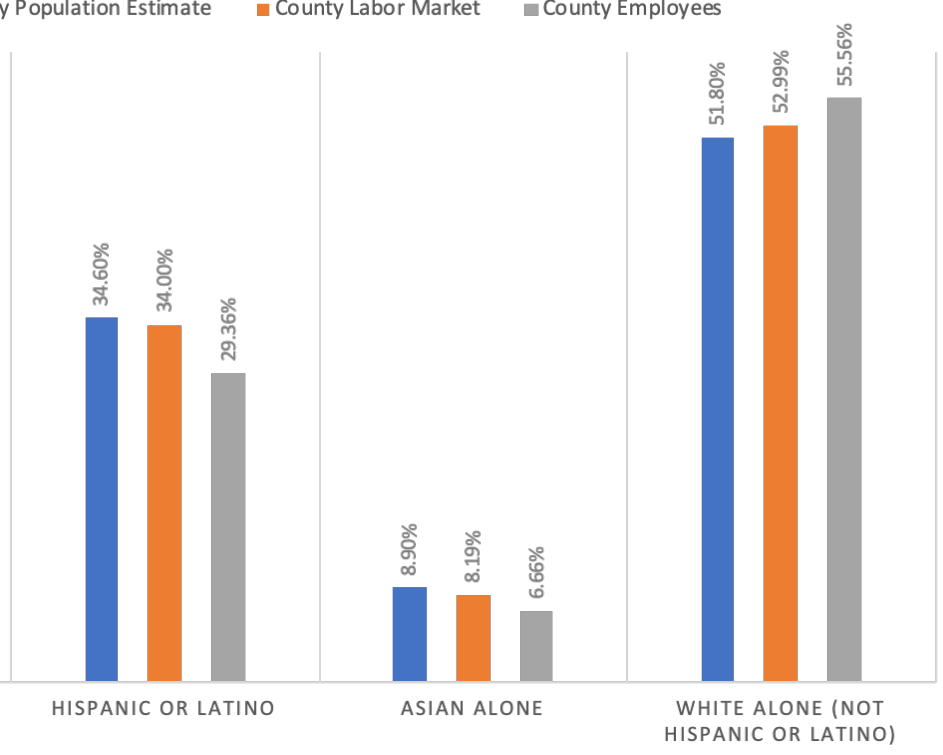

\section{NAPA COUNTY OFFICIALS AND ADMINISTRATORS}

- County Population Estimate = County Labor Market =County Employees

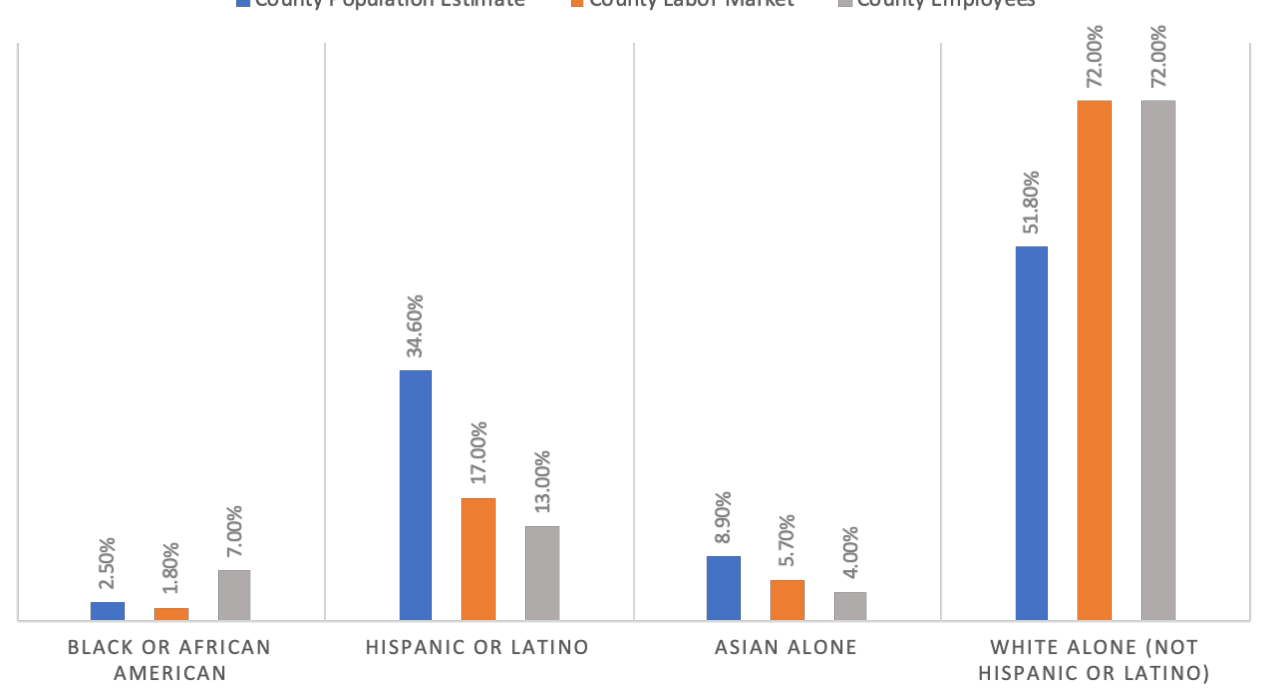




\section{Solano County}

Solano County is located in the northeastern part of the nine-county Bay Area region. The county has an overall population size of approximately 447,643 (US Census Bureau, 2021). There were a total of 3,141 allocated employee positions at the FY 20/21 Solano County budget hearings (B. Curry, personal communication, April 2, 2021).

Solano County currently does not have any diversity management programs due to the myriad of mandated programs that the county is dealing with. However, there has been some internal discussion about establishing a diversity management program (B. Curry, personal communication, April 2, 2021).

Diversity Management Program - Solano County SPECIAL EMPLOYEE BENEFITS

Employee Assistance Program (EAP)
"The County provides an Employee Assistance Program (EAP) through Anthem for employees and their eligible dependents. The EAP offers confidential, professional counseling services in a variety of areas such as, legal matters, marriage/family counseling, substance abuse, stress relief, and child care referrals. The EAP provides up to 5 counseling sessions per incident for employees and/or eligible dependents per fiscal year" (Solano County, n.d.).
Other Benefits
Dependent Care Reimbursement Account: "Employees may set aside up to $\$ 5,000$ pre-tax to pay for qualified childcare or dependent care expenses that are necessary for the employee and/or spouse to continue working" (Solano County, n.d.). 


\section{Workforce Representation Analysis - Solano County}

The following data is an analysis of the ethnicity of Solano County's 2021 workforce compared to the county's population and the labor market that is available countywide.

\section{$\underline{\text { Representation }}$}

- For 2021, White (47.19\%) employees have the highest representation among the four major ethnicities, followed by Hispanic or Latino $(21.10 \%)$, then Black or African American (14.94\%), and by Asian (8.92\%).

- White (+9.99\%) and Black or African American (+.14\%) are the two employee groups that have exceeded their representation with its overall population. However, the Hispanic or Latino group (-7.1\%) and Asian group (-9.3\%) are underrepresented.

- For the Officials and Administrators job group in Solano County, all three non-white employee groups are underrepresented, with Hispanics being the highest (-18.21\%).

\section{$\underline{\text { Utilization }}$}

- Similar to the overall population representation outcome, the Black or African American (+1.96\%) and White (+7.41\%) employee groups have exceeded their representation in the available workforce. On the other hand, Hispanic/Latino (-4.11\%) and Asian $(-7.15 \%)$ are both underutilized.

- Regarding representation in leadership positions, the white (64.85\%) employee group has the highest representation and is overutilized compared to its available local labor market for Officials and Administrators. The Black and African American employee group has also overutilized their available labor market officials and administrators within the region.

- Both Hispanic or Latino (-5.41\%) and Asian (-8.76\%) employee groups are underutilized for the Officials and Administrators category.
SOLANO COUNTY WORKFORCE
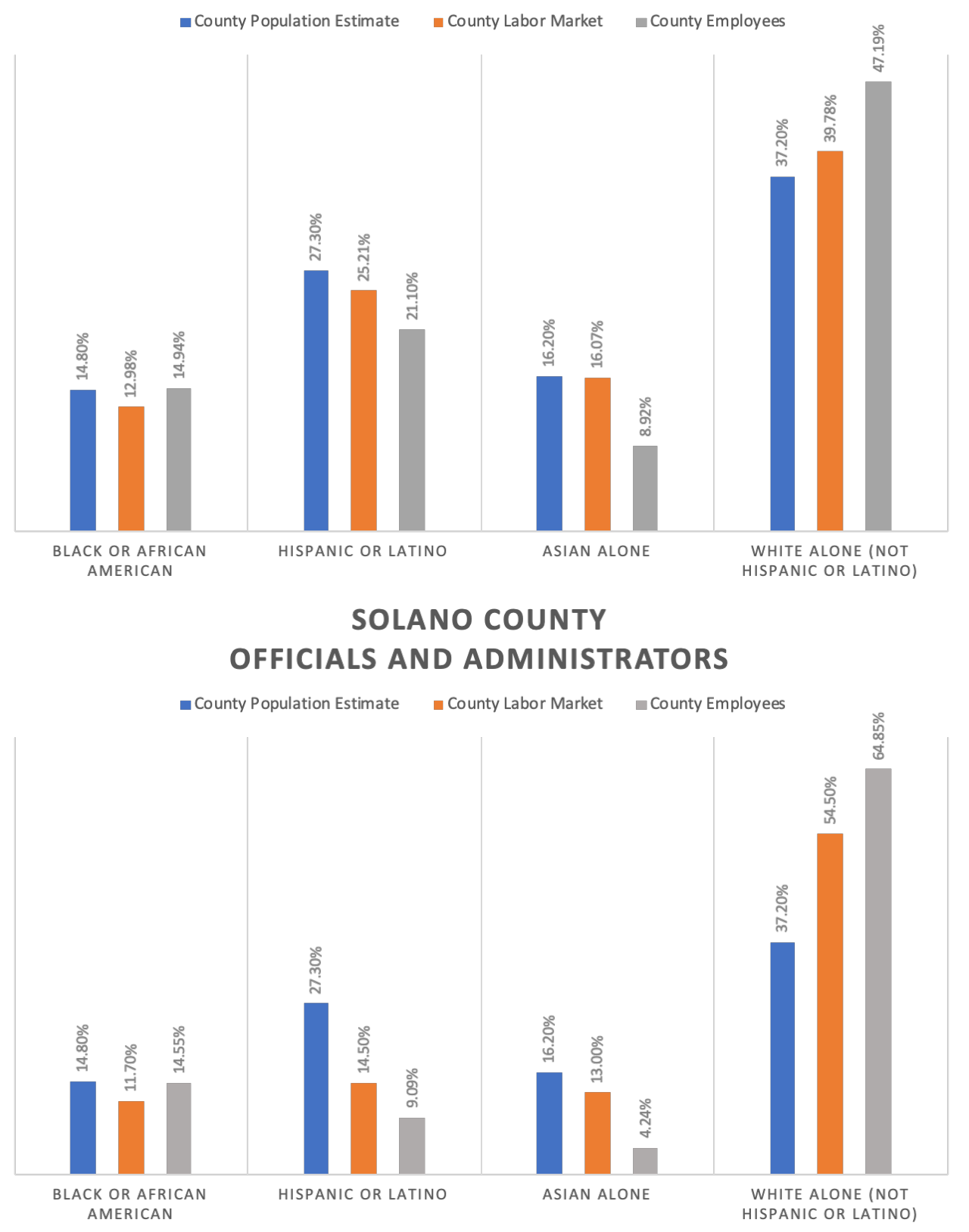


\begin{abstract}
ANALYSIS
This research focuses first on examining what diversity management programs and policies are currently implemented or planned by the nine Bay Area county government organizations and how they compare to IPMA standards. The previous Findings section answered this question by presenting an extensive summary of all the diversity management programs and policies from each of the nine Bay Area county government organizations. This section will now discuss these programs, and highlight the unique and popular strategies implemented within these jurisdictions.
\end{abstract}

Second, this research also intends to measure the representativeness of each of the nine counties' government organizations compared to the population they are serving. The study measured this by comparing the most recent workforce data collected from each county organization against the most current population data from the U.S. Census Bureau QuickFacts website. This section will interpret the overall findings and compare the reported trends of underrepresentation, particularly with minority work-groups.

Finally, as an additional method of measuring representation, this research seeks to determine whether underutilization of its available labor force supply occurs within this jurisdiction. This was measured by comparing each county government organization's most recent workforce data against each county's general labor market taken from the U.S. Census. This section will interpret the overall findings and trends of utilization in the Bay Area.

BP 1: The development of a formal diversity process that is contained in laws, rules, or procedures. Both human and financial time and resources are devoted to the program. In best 
practice organizations, diversity is a process that is an integrated, ongoing, and measurable strategy.

The need to have a bureaucracy that is representative of the population that it is serving is evident in the majority of the stated objectives for developing each of the county organizations' diversity management programs. The common focus among these county policies is the goal of attaining organizational efficiency by retaining an engaged and diverse workforce that can effectively and equitably respond to the needs of its rapidly diversifying clientele. These policies are effectuated and formalized with the intent to create a public organization that is tuned to the wellbeing of both its workforce and its constituents. Many of these plans are stand-alone diversity management programs, such as San Francisco's Executive Directive 18-02 (City and County, 2018), San Mateo's Diversity and Inclusion Strategic Initiative (County of San Mateo, 2017), Santa Clara's Board of Supervisors' Policy on Diversity (County of Santa Clara, 1993) and Alameda County's Diversity Program (Alameda County, 2021). Others are embedded in the organization's overall strategic plans, intended to improve the organization's way of doing business, such as Marin County's 5-Year Business Plan (Marin County, 2015) and Racial Equity Action Plan (Marin County, 2017), and Sonoma's 5-Year Strategic Plan (County of Sonoma, 2021).

Most of the mandated diversity initiatives contain strategic plans and timelines backed with Board of Supervisors approved funding, with delegated responsibilities assigned to staff at every level of the organization. Most of these diversity efforts are overseen by departments such as Human Resources, Equal Employment Opportunity, or newly established offices dedicated to promoting racial equity within and outside the organization. In addition to recruitment strategy enhancements, most diversity plans focus on strategies to promote cultural competency, 
inclusion, workforce development, and equity. Diversity policies have existed as early as 1993 (Santa Clara); however, the establishment of actionable diversity management initiatives was only formalized within the last five years.

\section{Drivers of Diversity}

In addition to the intent to diversify their organization's workforce, counties such as Marin, San Francisco, and Santa Clara were driven to create diversity management policies due to their workforce's demand in wanting a more inclusive and equitable working environment. The creation of diversity management initiatives is also due to the reignited sentiment to eradicate the growing racial disparities due to systemic issues, particularly in service delivery and internal practices, made salient by the nation's recent revolution for equity and social justice (e.g., Black Lives Matter movement) (Marin County, 2017c; Office of Racial Equity, n.d.; C. Cramer, personal communication, February 5, 2021; Contra Costa County, 2017; J. Mills, personal communication, February 2, 2021).

\section{GARE}

The Government Alliance on Race and Equity (GARE) has also been influential in the development of these racial equity action plans. Counties such as Marin, Santa Clara, Sonoma and Contra Costa have been sending cohorts of select employees since 2016 to participate in GARE facilitated training programs that assist them in developing plans and toolkits that will help them in ensuring racial equity within and outside their organization. GARE is a joint project of Race Forward and the Othering and Belonging Institute, which created a national network of governments working hand in hand to promote racial equity and the advancement of opportunities for all (Government Alliance on Race, 2021). 


\section{Organizational Readiness}

To ensure the successful implementation of diversity management initiatives, organizations are advised to conduct preliminary work prior to implementation of the diversity management plans by introducing the workforce to the goals and objectives of the diversity initiatives and facilitating team building or goal setting exercises that will help the workforce better understand the existing challenges (Soni, 2000). Several county organizations, such as Marin and Sonoma, created focus groups that not only included its workforce but also community members in the development of the plan (Marin County, 2020a; County of Sonoma, 2021b, p. 1). This will not only allow them to learn about and resolve internal diversity issues, but also receive the public's view on the policies, such as promoting equity in the recruitment processes.

BP 2: Diversity efforts are primarily decentralized, with a central governing body outlining the requirements of the plans, with individual agencies and departments developing their own plans that are tailored to their specific needs. This reinforces a sense of ownership and ensures that managing diversity has both top-level support and is a reality throughout the organization.

The City and County of San Francisco is the only jurisdiction among the nine county organizations that has adopted the decentralized approach in developing diversity plans. The Office of Racial Equity serves as its central governing agency. Alternatively, county organizations such as Marin and San Mateo have representatives from each participating department who collaborate with the county's leadership (e.g., County Administrator, HR Director, EEO Manager) and committee members or SMEs in developing and delivering countywide diversity plans. A decentralized model, wherein agencies and departments can autonomously implement and develop strategies according to the standards set by the central governing body, could be an ideal alternative for larger organizations. This model would allow 
central agencies to delegate the decision-making to different departments and agencies, making program delivery more efficient. Departments and agencies can each develop and implement their unique strategies to address their unique diversity challenges. The City and County of San Francisco has 35,557 employees, whereas Marin and San Mateo have 2,075 and 6,882 each, respectively. The majority of the organizations selected as IPMA's best practice organizations that incorporated the decentralized model are much larger state agencies, rather than local government agencies.

BP 3: In best-practice organizations, diversity training is provided to the workforce. The training is not limited to managers but is extended throughout the workforce. Successful organizations incorporate diversity into mentoring efforts, leadership training, and management-by-results programs.

Various iterations of diversity training are made available to the employees, some of which are required, and some are not. Most diversity training that is mandatory and recurring is generally meant for supervisors and managers only. Popular topics in these trainings include racial equity, inclusion, and structural/institutional racism. Previous research indicates that topics in diversity workshops should be relatable to the audience to be most effective (Rice, 2010). Marin County hosts forums and talks that focus on relevant national issues, while Contra Costa includes case studies that are relevant to the jobs of its audience in its mandatory training.

\section{Cultural Competency Training}

As noted earlier, a culturally competent workforce is needed to execute active representation fully (Rice, 2010). Counties such as Marin have a robust cultural competency training program easily made available to all employees. As of December 2020, 97\% of its employees have already completed the program (Muse, 2020). 


\section{Conflict Resolution Strategies}

Conflicts due to misunderstandings are common issues among diverse workgroups (Klinger, et al., 2010). The City and County of San Francisco and San Mateo County incorporate conflict management training in leadership and diversity training. San Francisco is planning on expanding and enhancing the curriculum of training and resources for its Skelly officers to help them better identify incidents of race-based biases in disciplinary actions (City and County, 2020, p. 35). Skelly Review Officers are impartial or uninvolved employees within the same public organization responsible for "evaluating whether there are reasonable grounds for believing that the employee engaged in the alleged misconduct and whether the misconduct supports the proposed sanction" (Simas, 2019, p. 1). Additionally, San Francisco has created a Citywide Peer Mediation program that trains employees to fix minor conflicts that are not considered EEO complaints (City and County, 2020, p. 50). This peer-to-peer mediation program is an excellent example of a non-adversarial dispute resolution technique that has proven to be a better approach in conflict resolution, particularly in a diverse working environment (Klingner, et al., 2010).

\section{Cross Cultural Communication}

Miscommunication or communication breakdown is another common issue experienced by a diverse workforce (Rice, 2010; Klinger, et al., 2010). The City and County of San Francisco has its own dedicated cross-cultural communication program to help employees develop strategies and styles to mitigate its effect (City and County, n.d.-h).

\section{Implicit Bias Training}

Most county organizations that have established diversity plans have mentioned their awareness of the inequitable effect of implicit bias in recruitment processes or the provision of employment 
opportunities in general. To address this issue, these jurisdictions have required their hiring managers and hiring panels (internal and external) to attend anti-bias training sessions, to provide them with strategies to increase their awareness of this phenomenon (City and County, 2018; Marin County, 2017b, p. 7; County of San Mateo, 2017b, p. 41; Contra Costa County, 2020b, p. 14).

BP 4: Best practice organizations use workforce data and demographics to compare statistics reported for the civilian labor force. Occupations with under-utilization are identified, and goals are established to reduce the underutilization. All of the best-practice organizations add creative and innovative recruitment strategies that get results and set their programs apart.

Several organizations use underutilization reports, generally provided by their respective EEO Offices, that are incorporated in the development of their recruitment strategies. Proactive strategies, such as the one in Contra Costa, mandate departments to include this best practice by requiring them to develop their recruitment and outreach strategic plans whenever a severe underutilization is reported in their shop (Contra Costa County, 2017). In addition to the data on underutilization, jurisdictions such as San Francisco also consider data on migration patterns, and impact analysis of public policies, when planning their recruitment outreach.

For some county organizations, data analysis does not stop at just comparing their current workforce to their available labor pools. Some of the recruitment plans include continuous monitoring of the applicant demographic before, during, and after the recruitment to assess whether a particular recruitment plan is adversely impacting a specific group due to unintended barriers (Marin County, 2019b; County of San Mateo, 2020, p. 4; Contra Costa County, 2017). Counties such as Contra Costa even developed an Adverse Impact Review guide to assist their human resources analysts in monitoring such occurrences in their job exams. 


\section{Hiring Toolkits}

This research was able to gather a wealth of information on various diversity recruitment strategies that are being used in these county organizations. To memorialize and operationalize these strategies, counties such as Marin and San Francisco have developed diversity hiring toolkits that both HR and departments can use as a guide to successfully attract a diverse applicant pool in their recruitments (Marin County, 2019b).

\section{Popular Outreach Strategies}

Some of the popular outreach strategies mentioned across the nine jurisdictions include participating in job fairs and community events, targeted advertising, coordinating with schools and professional organizations, and collaborating with diverse organizations on recruitment. Most of the nine Bay Area county organizations' outreach strategies can also be found in their Equal Employment Opportunity Plan Utilization Reports, which the Department of Justice requires them to complete (Department of Justice, n.d.).

\section{Strategies that Promote Equity}

Several county organizations have incorporated strategies that create an equal playing field for all aspiring applicants to promote equity in the recruitment process. Such strategies include mitigating the effect of implicit bias through blind application screening, such as what is being done in Napa; ensuring diversity in the interview panel (e.g., requiring one man, one woman and one person of color); asking uniform questions to all interviewees, and; requiring to interview at least one woman and one person of color for leadership positions (City and County, 2020; Nicholson, 2019; County of Santa Clara, 2019a, p. 5; County of San Mateo, 2020, p. 4). One could argue that using gender and color as a basis on who to interview violates Proposition 209; 
however, this strategy only intends to widen the pool of applicants to be considered and is not meant to influence the hiring decision based on a candidate's protective traits.

Counties such as Marin, San Francisco, and Napa have updated their job postings by using terms and descriptions that are both neutral and inclusive. The county organizations of Marin and San Francisco have updated their jobs' minimum qualifications to make it less stringent so that it can cast a wider net for potential candidates. This will make recruitments more accessible, especially for those from disadvantaged minority communities who may not have a strong background but sufficient experience to compete. San Francisco has also made their job examinations easier to understand and allowed make-up examinations for special reasons such as religious observance (City and County, 2020). Another equitable program that the City and County of San Francisco is known for is their Conviction History Program that allows all applicants, regardless of conviction history, to get an equal chance to compete for employment opportunities (City and County, 2020).

\section{Developing a Pipeline}

Another effective strategy that has helped several county organizations diversify their workforces is the creation of a worker's pipeline through internship programs. Counties such as Marin, San Francisco and San Mateo have opened their internship programs, not only for regular students, but also for other youths, or people in general, who are affected by socioeconomic inequities, or those who went through life experiences that generally bar them from getting employed (Marin County, 2018; Opportunities for All, n.d.; County of San Mateo, 2017b). These include youth with a juvenile justice system involvement, foster youth, or people who did not get the support needed to pursue an education. Participants in these programs get the benefits of a 
regular job and the support, training and education they need to grow, not only within the county but also in life.

\section{Leveraging Technology in Promoting Equity}

Several counties have also used technology as a tool to facilitate a more equitable recruitment process. Counties such as San Francisco are using a software called Textio that helps enhance job descriptions to use inclusive languages. Social media recruiting and video conferencing technology (Zoom), used in county organizations such as San Francisco and San Mateo, have opened the possibility for remote recruitment outreach that can easily be accessed by a wider audience (County of San Mateo, 2017b). Counties such as San Mateo have also expressed their intention to expand the online testing option, especially for those who cannot come onsite (County of San Mateo, 2017b).

BP 5: Best practice agencies have found that requiring diversity programs through the law, executive order, or other mandates compels agencies to establish serious goals and make earnest efforts toward meeting those goals.

Most diversity programs were effectuated through the Board of Supervisors' mandates and executive directives. The importance of leadership support and countywide participation is also stated numerous times as a critical element in these plans' successes. Each plan assigns specific goals and strategies to administrators, departments, department heads, workgroups, and committees. County organizations such as San Francisco require all departments to create racial equity action plans, which also have the element of diversity management (City and County, 2019). Contra Costa also requires departments to develop recruitment and outreach strategic plans when underutilization is reported (Contra Costa County, 2017). Unlike some examples 
cited in the original IPMA Best Practice article, there are no extreme consequences for failing to comply or meet certain goals in the Bay Area plans.

BP 6: Best practice organizations have established a review committee responsible for establishing policies, providing technical assistance, reviewing/approving plans, and monitoring progress toward achieving goals.

A few county organizations, such as Marin, San Mateo, Sonoma, and Contra Costa, have established their committees with varying oversight and responsibilities (Muse, 2020; County of San Mateo, n.d.-b; County of Sonoma, 2021b; Contra Costa County, 2021a). Most of these are limited to functions such as providing technical assistance, monitoring and evaluating plans and providing feedback and recommendations. The role of establishing and approving policies generally falls on the consensus of the leadership team, such as EEO Manager or Equity leaders.

Also, in the spirit of inclusivity, the County of Marin has opened the membership on its newly established committee - known as Workforce Equity Champions or WE Champions - to the public, encouraging the participation of representatives from diverse groups and underserved populations (Muse, 2020).

BP 7: Effective diversity programs also link recruitment, development, and retention strategies to organizational performance. They integrate employee development processes and map career paths to see what critical skills are necessary to advance, then communicate these skills to employees and provide training.

Although most counties have various career development programs, some programs are more proactive than others. Some of the popular employee development strategies include 
management development programs, such as supervisor and leadership training academies, published career maps, or ladders of different career classifications, to help employees navigate their career growth within the organization (Nicholson, 2019; Muse, 2020; Marin, n.d.; City and County, 2020; County of San Mateo, 2017b; Alameda County, 2021c). In addition to published career tracks, county organizations such as San Francisco and San Mateo have created Career Development Assessment Centers and a Career Development Advisors program to advise their employees (City and County, 2020; County of San Mateo, 2017b). Stretch assignments, acting roles, and working out of class opportunities are open to all employees interested in gaining experience working in higher roles (Nicholson, 2019; City and County, 2020; County of San Mateo, 2017b). In addition to mentorship programs, supervisors are also trained to develop coaching skills and provide feedback to their subordinates (Marin, 2015). Performance management tools and systems such as TalentQuest (Marin, 2015) and CPMS (County of San Mateo, 2017b) are also available for supervisors and regular employees. County organizations, such as Marin and San Francisco, have also partnered with local colleges to develop customizable degrees and certificate programs, not only for their employees but also community members who are interested in pursuing a career within the organization (e.g., Marin County's Firefighter Pilot Program) (Muse, 2020; Marin County,2015; City and County, n.d.-e). Not only is it a good career development program, but it also creates an equitable career pipeline, especially for disadvantaged members of the community.

BP 8: Accountability for the results of diversity programs is another attribute of best-practice organizations. Accountability is determined through metrics, surveys, focus groups, customer surveys, management, employee evaluations, and training and education evaluations. 
In addition to the underutilization reports that most county EEO offices track, which could give the overall impact analysis of diversity efforts, counties such as Marin and San Francisco also keep track of demographic trends in the employment lifecycle - from hiring to separations (Marin County, 2020c). Data collection tools such as ATS are being used for data monitoring (City and County, 2020). In addition, they also monitor diversity trends in the candidate pool, promotions, and employee discipline to monitor for potential disparities (City and County, 2020).

Qualitative data is also collected before and after the execution of each diversity action plan. The County of Sonoma plans on conducting employee engagement and racial equity awareness surveys to serve as their baseline for their diversity management strategies (County of Sonoma, 2021b). Marin County also collects data to monitor the effectiveness of its training and employee satisfaction through focus groups (e.g., Possibility Posse) and surveys (Muse, 2020).

County organizations of Marin, Santa Clara, and San Francisco also express accountability by sharing their workforce demographic data and pay equity reports with the public through their websites (Marin County, 2021; County of Santa Clara; 2020; City and County, n.d.-k). The public's feedback is also deemed critical in the development and improvement of diversity efforts, particularly in recruitment, which is why counties such as San Francisco and Marin also invite community partners to share their insights (Muse, 2020; City and County, 2020). 


\section{Unique Diversity Management Strategies}

\section{Initiatives About Inclusion and Belonging}

Although the strategies that promote inclusion and belonging are not heavily emphasized in IMPA's list of best practices, most Bay Area counties are following the recent trend of incorporating inclusion and belonging in their diversity strategies. Both Marin and San Francisco have formed Affinity Groups composed of different ethnic subgroups that are coming together to promote cross-cultural understanding and inclusivity (City and County, 2020; Marin County, 2019c). Also, since the feeling of inclusion manifests when a person or a group of people feel that they are being heard, supported, and included, counties such as Marin facilitate group sessions, such as Safe Space Brave Space, that not only discuss contemporary racial issues but also give attendees a venue to discuss their opinions and workplace concerns (Muse, 2020). Other inclusion and belonging strategies found in these counties include recognition of religious and cultural holidays and considering them for paid time offs, offering awards to recognize and encourage programs that foster diversity and inclusion, and creating an art décor committee that ensures that all artworks and decor within county facilities reflect the diversity of the organization (City and County, 2020; County of San Mateo, 2017b; City and County, 2020).

\section{Initiatives About Equity of Opportunities and Pay}

As a testament to their commitment to an equitable provision of benefits and opportunities, counties such as Santa Clara and San Francisco have implemented policies that monitor and ensure equity in pay for all employees regardless of race and gender (City and County, 2020). The County of Santa Clara has even created a publicly available dashboard on their website that presents this data in real-time (County of Santa Clara; 2020). 


\section{Special Employee Benefits}

\section{Unique Benefits (e.g., leaves, child and elder care support, flexible work locations, EAP)}

As mentioned in this research, one of the diversity management strategies that HRM uses to promote and sustain diversity in the workforce is through benefit policies.

All nine Bay Area counties have versions of Employee Assistance Programs (EAP) that benefit employees and their families. Popular EAP services include counseling services, legal and financial advice, and work/life balance support (Marin County, 2019d; San Francisco Health, 202; County of San Mateo, n.d.-c; County of Sonoma, 2021f; Contra Costa County, 2021b; County of Santa Clara Employee Assistance, 2021; Alameda County, 2021d; Napa County, 2021; Solano County, n.d.).

Since housing and commuting are significant challenges for Bay Area residents, several county organizations offer commuter subsidies and benefits to help employees reduce the cost of commuting (Marin County, 2019e; Contra Costa, 2021f; County of Santa Clara Employee Services, 2021b). The County of Marin is also piloting an employee housing assistance program to assist its employees with the initial cost of relocating closer to their workplace (Marin County, 2019e). As an alternative solution to commuting, Sonoma and San Mateo counties encourage departments to explore teleworking options to promote employee work/life balance (County of Sonoma, 2021b, p. 11; County of San Mateo, 2017b). It has been said that "when a company has a commuter benefits program in place, it communicates to employees that the organization is invested in them and wants them to be happy at their jobs" (SHRM, 2019, p. 1). 
As mentioned earlier in this paper, diversity management programs are not only meant to promote and maintain racial diversity. Certain benefits are also aimed at promoting diversity in gender. Some employee benefits offered in the nine Bay Area county organizations popular with women include the flexible provision of leaves and paid time off for childcare, parental and sibling care (City and County, n.d.-i; County of San Mateo, 2017b). In addition, Bay Area county organizations also offer Dependent Care Assistance Programs or DCAP, a tax-advantage benefit which can help workers in cutting down the cost of dependent care (e.g. children under 13, care for physically and mentally disabled dependents) (Contra Costa County, 2021d; County of Santa Clara Employee Services, 2021a; Alameda County, 2021e; Solano County, n.d.). County organizations such as Contra Costa and Alameda also offer child daycare services options to their employees (Alameda County, 2021f; Contra Costa County, 2021c).

Another HRM diversity management program mentioned in this research is identifying and preventing harassment. Sexual harassment trainings are now required by law in California, and this research confirmed that all nine Bay Area county organizations are compliant with this requirement. Supervisors and non-supervisory employees must take the training (County of San Mateo, n.d.-d). 
Table 1 summarizes the best practices currently implemented in each county, the year when they were established, and the current size of their workforce.

Table 1: Summary of Best Practices in Bay Area Counties

\begin{tabular}{|c|c|c|c|c|c|c|c|c|c|}
\hline & Marin & San Francisco & San Mateo & Sonoma & Contra Costa & Santa Clara & Alameda & Napa & Solano \\
\hline BP1 & $V$ & $V$ & $V$ & $V$ & $\checkmark$ & $V$ & & & \\
\hline BP2 & & $V$ & & & & & & & \\
\hline BP3 & $\checkmark$ & $\checkmark$ & $\checkmark$ & $\checkmark$ & $\checkmark$ & $\checkmark$ & & & \\
\hline BP4 & $\checkmark$ & $\checkmark$ & $\checkmark$ & $\checkmark$ & $V$ & & & $V$ & \\
\hline BP5 & $\checkmark$ & $V$ & $V$ & $\checkmark$ & $V$ & & & & \\
\hline BP6 & $\checkmark$ & & $\checkmark$ & $\checkmark$ & $\checkmark$ & & & & \\
\hline BP7 & $V$ & $\checkmark$ & $V$ & $\checkmark$ & & & $\checkmark$ & & \\
\hline BP8 & $\checkmark$ & $V$ & $V$ & $\checkmark$ & $\checkmark$ & & & & \\
\hline Year Plan Started & 2015 & 2018 & 2014 & 2021 & 2017 & 1993 & NA & NA & NA \\
\hline $\begin{array}{c}\text { Size of } \\
\text { Organization }\end{array}$ & $2075 \mathrm{FTE}$ & 35,557 FTE & $6,882 \mathrm{FTE}$ & 3,855 FTE & 10,748 FTE & 17,185 FTE & 8,296 FTE & 1,366 FTE & 3,141 FTE \\
\hline
\end{tabular}

Source: The organization's size was either taken from the county's online workforce dashboard or requested via Public Records Request. 


\section{Bay Area Population Breakdown}

Figure 1 shows the nine Bay Area county organizations' most recent population estimates by percentage of the population, broken down by the four major ethnicities, taken from the U.S. Census QuickFacts website. Solano is recorded to have the highest percentage of Black or African American residents (14.80\%) in the population, Napa has the highest percentage of Latino or Hispanic residents $(34.60 \%)$ in the population, Santa Clara has the highest percentage of Asian residents (39\%) in the population, which is also the largest racial group within the jurisdiction, as it is in Alameda County, where Asian residents represent (32.30\%) of the population. Marin County is the jurisdiction that has the highest percentage of white residents $(71.10 \%)$ in the population.

Figure 1: Population of the Nine Bay Area Counties by Race and Ethnicity

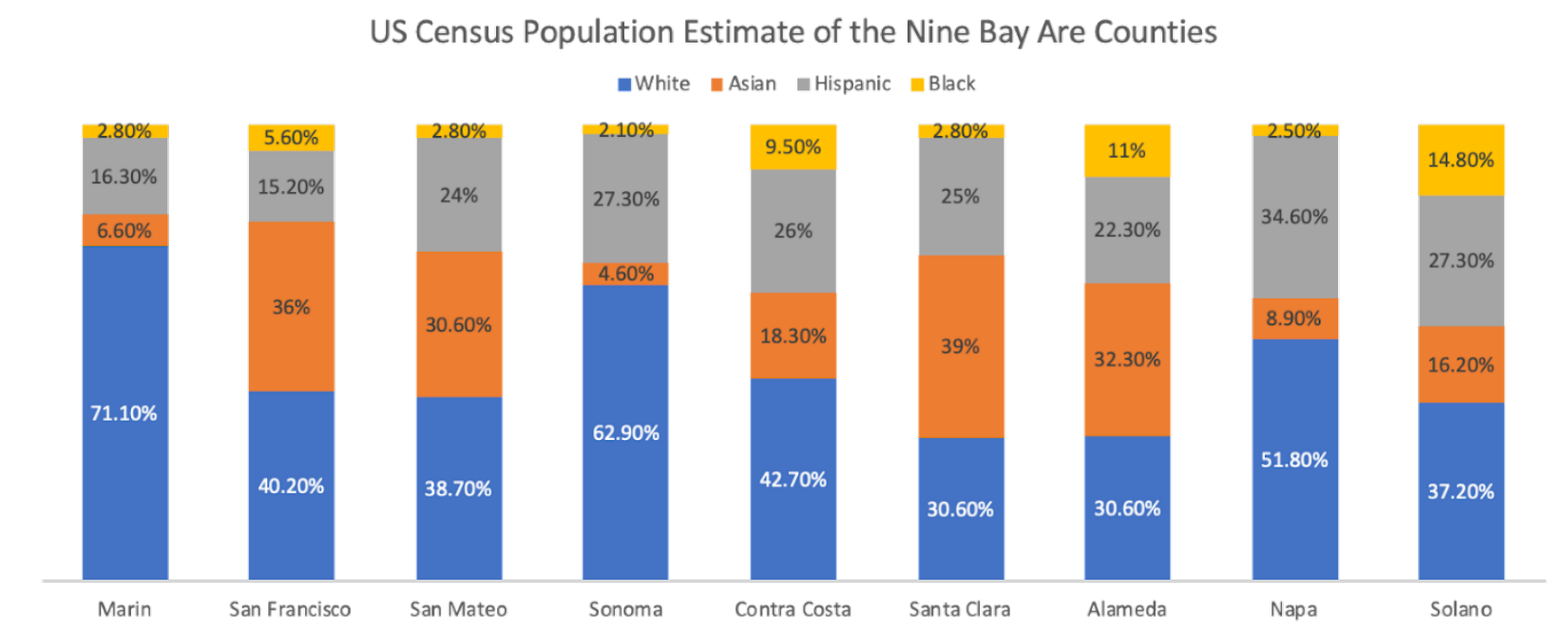

Source: [The county population estimates were taken from the U.S. Census Bureau Quick Facts portal. These are estimates as of July 1,2019 .] 
Table 2: Nine Bay Area Counties’ Workforce By County By Race

\begin{tabular}{|c|c|c|c|c|c|c|c|c|c|}
\hline & Marin & San Francisco & San Mateo & Sonoma & Contra Costa & Santa Clara & Alameda & Napa & Solano \\
\hline Total County Population & 258,826 & 881,549 & 766,573 & 494,336 & $1,153,526$ & $1,927,852$ & $1,671,329$ & 137,744 & 447,643 \\
\hline Total Employee Count & 2075 & 35,557 & 6882 & 3855 & 10748 & 17,185 & 8296 & 1366 & 3,141 \\
\hline County Employees & 2020 & 2020 & 2021 & 2021 & 2021 & 2021 & 2019 & 2021 & 2021 \\
\hline Black or African American & $6.50 \%$ & $15.13 \%$ & $4.9 \%$ & $2 \%$ & $14.50 \%$ & $5.20 \%$ & $25 \%$ & $4.83 \%$ & $14.94 \%$ \\
\hline Hispanic or Latino & $18.90 \%$ & $15 \%$ & $21.80 \%$ & $21 \%$ & $19.28 \%$ & $26.70 \%$ & $17 \%$ & $29.36 \%$ & $21.10 \%$ \\
\hline Asian alone & $9.90 \%$ & $39.80 \%$ & $24.6 \%$ & $3 \%$ & $12.78 \%$ & $32.00 \%$ & $23 \%$ & $6.66 \%$ & $8.92 \%$ \\
\hline White Alone, not Hispanic or Latino & $60.60 \%$ & $28.57 \%$ & $27.60 \%$ & $68 \%$ & $37 \%$ & $19.50 \%$ & $30 \%$ & $55.56 \%$ & $47.19 \%$ \\
\hline \multicolumn{10}{|c|}{ US Census Population Estimate (July 1, 2019) } \\
\hline Black or African American & $2.80 \%$ & $5.60 \%$ & $2.80 \%$ & $2.10 \%$ & $9.50 \%$ & $2.80 \%$ & $11.00 \%$ & $2.50 \%$ & $14.80 \%$ \\
\hline Hispanic or Latino & $16.30 \%$ & $15.20 \%$ & $24.00 \%$ & $27.30 \%$ & $26 \%$ & $25 \%$ & $22.30 \%$ & $34.60 \%$ & $27.30 \%$ \\
\hline Asian alone & $6.60 \%$ & $36.00 \%$ & $30.60 \%$ & $4.60 \%$ & $18.30 \%$ & $39 \%$ & $32.30 \%$ & $8.90 \%$ & $16.20 \%$ \\
\hline White Alone, not Hispanic or Latino & $71.10 \%$ & $40.20 \%$ & $38.70 \%$ & $62.90 \%$ & $42.70 \%$ & $30.6 \%$ & $30.60 \%$ & $51.80 \%$ & $37.20 \%$ \\
\hline \multicolumn{10}{|c|}{ Underrepresentation (County Employees) } \\
\hline Black or African American & $3.70 \%$ & $9.53 \%$ & $2.10 \%$ & $-0.10 \%$ & $5.00 \%$ & $2.40 \%$ & $14.00 \%$ & $2.33 \%$ & $0.14 \%$ \\
\hline Hispanic or Latino & $2.60 \%$ & $-0.20 \%$ & $-2.20 \%$ & $-6.30 \%$ & $-6.72 \%$ & $1.70 \%$ & $-5.30 \%$ & $-5.24 \%$ & $-6.20 \%$ \\
\hline Asian alone & $3.30 \%$ & $3.80 \%$ & $-6.00 \%$ & $-1.60 \%$ & $-5.52 \%$ & $-7.00 \%$ & $-9.30 \%$ & $-2.24 \%$ & $-7.28 \%$ \\
\hline White Alone, not Hispanic or Latino & $-10.50 \%$ & $-11.63 \%$ & $-11.10 \%$ & $5.10 \%$ & $-5.70 \%$ & $-11.10 \%$ & $-0.60 \%$ & $3.76 \%$ & $9.99 \%$ \\
\hline \multicolumn{10}{|l|}{ Census 2014-18 EEO FTP } \\
\hline Black or African American & $1.80 \%$ & $3.83 \%$ & $2.10 \%$ & $1.40 \%$ & $8.26 \%$ & $2.48 \%$ & $9.82 \%$ & $1.90 \%$ & $12.98 \%$ \\
\hline Asian alone & $6.30 \%$ & $31.78 \%$ & $29.94 \%$ & $4.24 \%$ & $16.68 \%$ & $36.41 \%$ & $29.65 \%$ & $8.19 \%$ & $16.07 \%$ \\
\hline White Alone, not Hispanic or Latino & $73.25 \%$ & $45.54 \%$ & $39.10 \%$ & $64.73 \%$ & $45.55 \%$ & $33.00 \%$ & $34.50 \%$ & $52.99 \%$ & $39.78 \%$ \\
\hline \multicolumn{10}{|l|}{ Underutilization (County Employees) } \\
\hline Black or African American & $4.70 \%$ & $11.30 \%$ & $2.80 \%$ & $0.60 \%$ & $6.24 \%$ & $2.72 \%$ & $15.18 \%$ & $2.93 \%$ & $1.96 \%$ \\
\hline Hispanic or Latino & $3.56 \%$ & $0.44 \%$ & $-2.29 \%$ & $-4.61 \%$ & $-5.20 \%$ & $1.98 \%$ & $-4.09 \%$ & $-4.64 \%$ & $-4.11 \%$ \\
\hline Asian alone & $3.60 \%$ & $8.02 \%$ & $-5.34 \%$ & $-1.24 \%$ & $-3.90 \%$ & $-4.41 \%$ & $-6.65 \%$ & $-1.53 \%$ & $-7.15 \%$ \\
\hline White Alone, not Hispanic or Latino & $-12.65 \%$ & $-16.97 \%$ & $-11.50 \%$ & $3.27 \%$ & $-8.55 \%$ & $-13.50 \%$ & $-4.50 \%$ & $2.57 \%$ & $7.41 \%$ \\
\hline
\end{tabular}

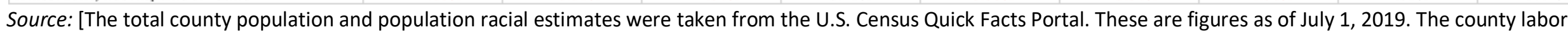
market data was taken from California's 5-Year EEO Tabulation Report by county and state from 2014-2018 requested from the U.S. Census Bureau.]

Note: Underrepresentation is calculated by subtracting the U.S. Census population estimate from county employees for each race. Underutilization is calculated by subtracting the county labor market data (Census 2014-18 EEO) from county employees for each race. 
Table 3: Nine Bay Area Counties’ Workforce By County By Race for Officials and Administrators

\begin{tabular}{|c|c|c|c|c|c|c|c|c|c|}
\hline & Marin & San Francisco & San Mateo & Sonoma & Contra Costa & Santa Clara & Alameda & Napa & Solano \\
\hline Officials and Administrators (County) & 2020 & 2020 & 2021 & 2021 & 2021 & 2021 & 2019 & 2021 & 2021 \\
\hline Black or African American & $12.00 \%$ & $11.59 \%$ & $5.10 \%$ & $3.00 \%$ & $13.84 \%$ & $3.74 \%$ & $26.84 \%$ & $7 \%$ & $14.55 \%$ \\
\hline Hispanic or Latino & $10.20 \%$ & $7.93 \%$ & $18.30 \%$ & $6 \%$ & $11.58 \%$ & $5.88 \%$ & $13.42 \%$ & $13 \%$ & $9.09 \%$ \\
\hline Asian alone & $14.00 \%$ & $20.73 \%$ & $22.10 \%$ & $1 \%$ & $8 \%$ & $37.97 \%$ & $14.09 \%$ & $4 \%$ & $4.24 \%$ \\
\hline White Alone, not Hispanic or Latino & $63.80 \%$ & $58.54 \%$ & $41.40 \%$ & $84 \%$ & $55 \%$ & $20.86 \%$ & $38.25 \%$ & $72 \%$ & $64.85 \%$ \\
\hline \multicolumn{10}{|c|}{ US Census Population Estimate (July 1, 2019) } \\
\hline Black or African American & $2.80 \%$ & $5.60 \%$ & $2.80 \%$ & $2.10 \%$ & $9.50 \%$ & $2.80 \%$ & $11.00 \%$ & $2.50 \%$ & $14.80 \%$ \\
\hline Hispanic or Latino & $16.30 \%$ & $15.20 \%$ & $24.00 \%$ & $27.30 \%$ & $26 \%$ & $25 \%$ & $22.30 \%$ & $34.60 \%$ & $27.30 \%$ \\
\hline Asian alone & $6.60 \%$ & $36.00 \%$ & $30.60 \%$ & $4.60 \%$ & $18.30 \%$ & $39 \%$ & $32.30 \%$ & $8.90 \%$ & $16.20 \%$ \\
\hline White Alone, not Hispanic or Latino & $71.10 \%$ & $40.20 \%$ & $38.70 \%$ & $62.90 \%$ & $42.70 \%$ & $30.6 \%$ & $30.60 \%$ & $51.80 \%$ & $37.20 \%$ \\
\hline \multicolumn{10}{|l|}{ Underrepresentation (O\&A) } \\
\hline Black or African American & $9.20 \%$ & $5.99 \%$ & $2.30 \%$ & $0.90 \%$ & $4.34 \%$ & $0.94 \%$ & $15.84 \%$ & $4.50 \%$ & $-0.25 \%$ \\
\hline Hispanic or Latino & $-6.10 \%$ & $-7.27 \%$ & $-5.70 \%$ & $-21.30 \%$ & $-14.42 \%$ & $-19.12 \%$ & $-8.88 \%$ & $-21.60 \%$ & $-18.21 \%$ \\
\hline Asian alone & $7.40 \%$ & $-15.27 \%$ & $-8.50 \%$ & $-3.60 \%$ & $-10.40 \%$ & $-1.03 \%$ & $-18.21 \%$ & $-4.90 \%$ & $-11.96 \%$ \\
\hline White Alone, not Hispanic or Latino & $-7.30 \%$ & $18.34 \%$ & $2.70 \%$ & $21.10 \%$ & $12.30 \%$ & $-9.74 \%$ & $7.65 \%$ & $20.20 \%$ & $27.65 \%$ \\
\hline \multicolumn{10}{|l|}{ Census 2014-18 EEO FTP (O\&A) } \\
\hline Black or African American & $1.40 \%$ & $2.60 \%$ & $1.50 \%$ & $0.70 \%$ & $5.60 \%$ & $2.10 \%$ & $8.20 \%$ & $1.80 \%$ & $11.70 \%$ \\
\hline Hispanic or Latino & $5.80 \%$ & $8.50 \%$ & $11.10 \%$ & $12.4 \%$ & $12.80 \%$ & $13.10 \%$ & $11.50 \%$ & $17 \%$ & $14.50 \%$ \\
\hline Asian alone & $5.10 \%$ & $24.80 \%$ & $29.90 \%$ & $4.3 \%$ & $17 \%$ & $35.90 \%$ & $29.30 \%$ & $5.70 \%$ & $13.00 \%$ \\
\hline White Alone, not Hispanic or Latino & $84.70 \%$ & $60.00 \%$ & $53.60 \%$ & $79 \%$ & $59.60 \%$ & $45.90 \%$ & $46.80 \%$ & $72 \%$ & $54.50 \%$ \\
\hline \multicolumn{10}{|l|}{ Underutilization (O\&A) } \\
\hline Black or African American & $10.60 \%$ & $8.99 \%$ & $3.60 \%$ & $2.30 \%$ & $8.24 \%$ & $1.64 \%$ & $18.64 \%$ & $5.20 \%$ & $2.85 \%$ \\
\hline Hispanic or Latino & $4.40 \%$ & $-0.57 \%$ & $7.20 \%$ & $-6.40 \%$ & $-1.22 \%$ & $-7.22 \%$ & $1.92 \%$ & $-4.00 \%$ & $-5.41 \%$ \\
\hline Asian alone & $8.90 \%$ & $-4.07 \%$ & $-7.80 \%$ & $-3.30 \%$ & $-9.40 \%$ & $2.07 \%$ & $-15.21 \%$ & $-1.70 \%$ & $-8.76 \%$ \\
\hline White Alone, not Hispanic or Latino & $-20.90 \%$ & $-1.46 \%$ & $-12.20 \%$ & $5.00 \%$ & $-4.60 \%$ & $-25.04 \%$ & $-8.55 \%$ & $0.00 \%$ & $10.35 \%$ \\
\hline
\end{tabular}

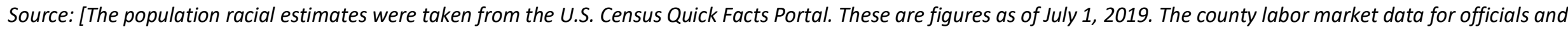
administrators were taken from California's 5-Year EEO Tabulation Report by county and state from 2014-2018 requested from the U.S. Census Bureau.]

Note: Underrepresentation is calculated by subtracting the U.S. Census population estimate from each county's number of officials and administrators for each race. Underutilization is calculated by subtracting the county labor market data of officials and administrators (Census 2014-18 EEO) from the number of officials and administrators of each county for each race. 


\section{Testing for Passive Representation}

As mentioned earlier, one of the ways that a bureaucracy can attain representativeness is through passive representation. Passive representation is achieved when the workforce of a public organization is able to mirror the demographic makeup of the community that it is serving. It is also said that this is the original intent of Affirmative Action and that its dissolution in California, with the passage of Proposition 209, has worsened the underrepresentation of minorities, particularly with the Latino or Hispanic minority group. This is particularly troubling because the Latino community is said to be the fastest growing minority group and could potentially become the largest minority group by the year 2050 (Riccucci, 2016; Passel \& Cohn, 2008). Rice (2010) also said that Asian Americans are significantly underrepresented in both state and local governments. Table 2 shows several instances of underrepresentation across the nine Bay Area county organizations, particularly with the Hispanic or Latino, Asian, and even the white employee groups.

Citizen status is often required for public sector jobs, which may be a barrier for Hispanics disproportionately from other races/ethnicities. A 2017 report done by Pew Research shows that Latinos, particularly Mexican lawful immigrants are least likely to consider U.S. citizenship compared to other races (Gonzales-Barrera, 2017). Furthermore, a high school diploma is a minimum requirement for most government jobs. The 2019 Census data on educational attainment in the United States shows that only $73 \%$ of Hispanics over 18 have a high school diploma, compared with $87 \%$ of Black, $91 \%$ of Asian, and $94 \%$ of the white population (U.S. Census, 2019). Because of these and other knowledge, skills, and abilities characteristics of the Hispanic population, a more meaningful statistical method, such as comparing applicants versus employees, may be useful in determining whether or not there is 
ethnicity-based prejudice against Hispanics in public employment. This data would also inform decisions on remedies that would provide more opportunities for Hispanic residents in the future, such as mentoring high school students toward earning a degree or enhancing their knowledge, skills, and abilities credentials.

Though the Asian employee group is underrepresented in several jurisdictions, they are well represented in Santa Clara and San Francisco - even more than the white employee group. Cultural standards may account for low Asian American representation in government employment. Parental and social pressure to enter more prestigious professions may deter Asians from applying for government jobs (Ip, 2013). This area would require more study, such as collecting data on applications versus employment, to determine whether Asian applicants are being discriminated against, or simply not in the hiring pool.

Although Black or African Americans have ended up at the bottom of nearly every category in the private sector (Adejumo, 2021), this group is doing relatively well in the public sector (Klingner, et al., 2010). As shown in Table 2, the Black or African American employee group is the only group within the nine county organizations that is consistently overrepresented in almost every jurisdiction, except for Sonoma County. This trend could be a testament to the continuous appeal of public sector jobs to the African American community (Riccucci \& Van Ryzin, 2017, p 22) or could be due to sampling reasons as a result of having a small percentage of Black or African American population in each of the Bay Area counties (Figure 1). A few number of employees may result in an "overrepresentation" statistically. 


\section{Testing for Active Representation}

To promote better policy making and more equitable administration of programs, bureaucracies must have diverse leaders in the ranks who can actively represent their counterparts in the community (Riccucci, 2009; Feeney \& Camarena, 2021). White employees are purported to be disproportionately the majority in the leadership category (Riccucci, 2016; Adejumo, 2021). In fact, Table 3 ascertains that the white employee group is still disproportionately the majority in the officials and administrators category in the nine Bay Area county organizations. Similar to what Holzer \& Neumark (1999) said, it can be argued that the white overrepresentation in the Bay Area leadership ranks may be remnants of historic hiring practices rather than current equity-informed practices. Further study could collect data on the length of service of employees by ethnicity to determine whether current practices are moving toward an employee group that better represents the community.

The Hispanic or Latino employee group has recorded underrepresentation in every single jurisdiction in the officials and administrators category, which is notable in Napa County (Table 3), which ironically has the most significant percentage of Hispanic and Latino residents among the nine county organizations (Figure 1). This again goes back to the prediction that Latinos could become the largest minority group in the country. According to the theory of representative bureaucracy, leaders that mirror the ethnicity of residents will be needed for active representation to work.

Even though Blacks or African Americans are generally well represented in the public sector, they are still underrepresented in the middle and upper management (Rice, 2010). Table 3 shows that the Black or African American employee group is overrepresented in almost all jurisdictions in the officials and administrators category, except for a slight underrepresentation 
in Solano County, where its population percentage is the highest (Figure 1). Alameda County recorded the highest significant overrepresentation of African Americans in the Officials and Administrators category and had the second-highest population of African Americans (Figure 1).

Although Asians are predominantly underrepresented across the nine county organizations, the Asian employee group in Santa Clara County is the only non-white employee group that has consistently surpassed the number of its white counterpart overall (Table 2) and so as in the Official and Administrators category (Table 3). Although this research cannot ascertain the correlation of having a majority Asian constituency with having an Asian-majority leadership in Santa Clara County, it could be that having diverse leaders could lead to hiring diverse employees. A County of Santa Clara employee has confirmed that this trend has only happened recently, since Asians in the Officials and Administrator category in 2017 were only 33\% of employees, while the white employee group during that time was at $44 \%$ of employees (P. Laprairie, personal communication, April 15, 2021). Though unproven, it may be possible that the white population numbers and percentage of residents in the county also dropped during this time.

Feeney \& Camarena (2021) said that “when a city's minority population increases, governments become more representative in political and bureaucratic leadership" (p. 123). As shown in Figure 1, Marin County has the least diverse population but the most representative of them all, with zero underrepresentation in the overall workforce (Table 2). 
20-Year Trend for Officials and Administrators (Figure 3)
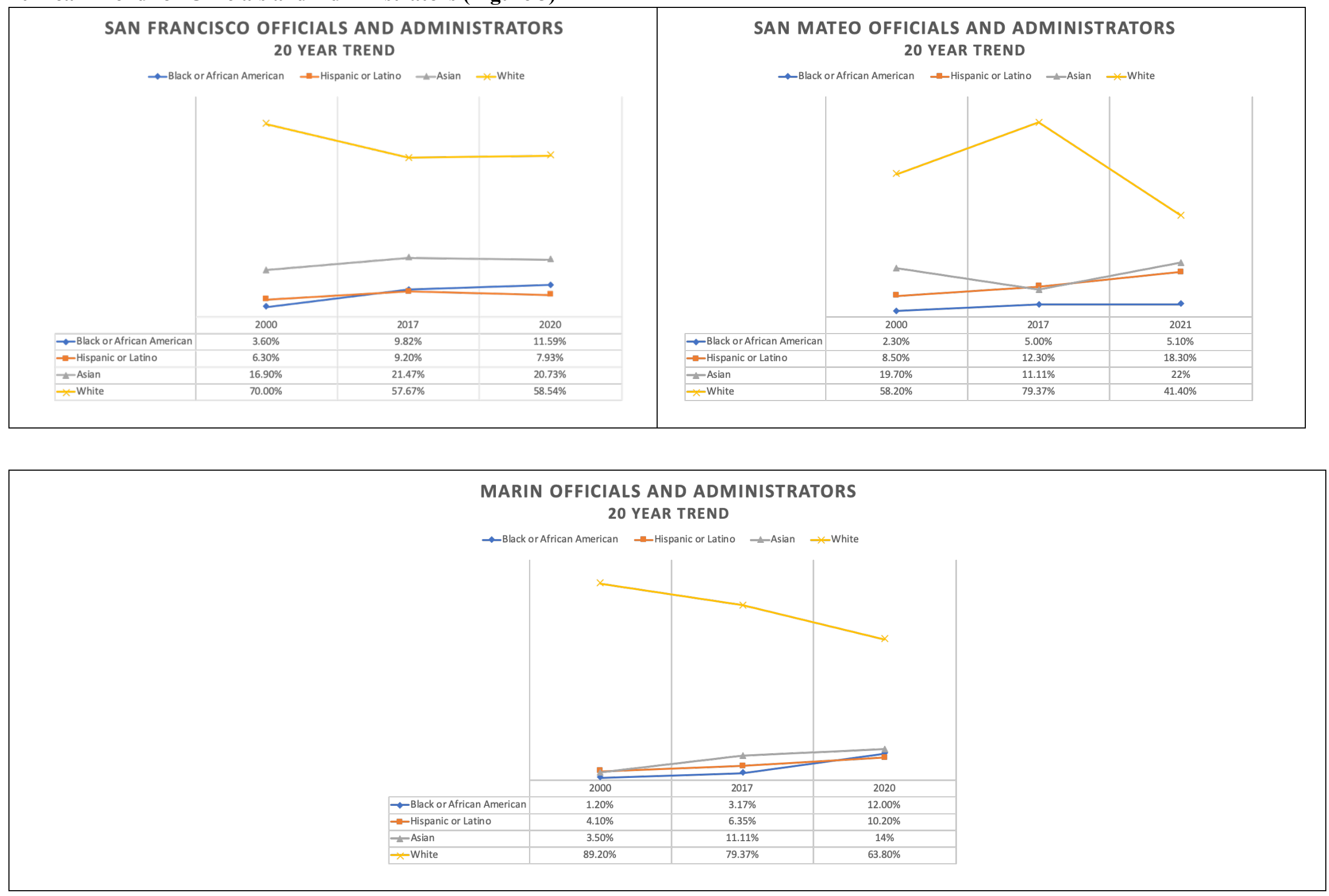

Source: Workforce data dashboard from the county website (Marin, San Mateo and San Francisco) 
Geshekter (2008) has shown the overall drop in white employees and increase of minority employees in the California public labor workforce, which he attributed to the public sector's significant strides in improving their process in recruiting a more diverse employee group. This has included concerns for equity in hiring. Marin, San Mateo, and San Francisco provided public employee data that allowed for comparing ethnicities in the county organization's employment. Comparing the 20-year trend for Marin, San Mateo, and San Francisco counties corroborates this trend in the nine county organizations (Figure 3). A similar pattern is seen showing the decrease of the white population in the nine county Bay Area region between 1980 to 2014, which may have also contributed to this trend (Bay Area Equity Atlas, 2017). The Bay Area's population has jumped from 5.1 million to 7.4 million, with its ethnic minority demographic increasing from $31 \%$ to $59 \%$ (Bay Area Equity Atlas, 2017).

\section{Workforce Underutilization}

When comparing the nine Bay Area county organizations' workforce with its available labor market, tables 2 and 3 show that the Hispanic or Latino, Asian, and white employee groups have all recorded a degree of underutilization. Data also shows that the Black or African American employee group is consistently overutilized across the nine Bay Area county organizations on the overall workforce (Table 2) and leadership (Table 3). Same as its record of having zero underrepresentation, Marin County recorded zero underutilization of ethnic groups for both its overall workforce (Table 2) and in leadership (Table 3). Its neighboring jurisdiction, the City and County of San Francisco, also recorded zero underutilization on the overall workforce (Table 2).

When creating workforce underutilization reports, the Department of Justice has said that the percent threshold for identifying significant underutilization could vary depending on the 
workforce's size being studied (Department of Justice, n.d.). For example, "having a 20 percent underutilization of Asian males in the Skilled Craft job category would be less significant for an employer who has a total of five employees in this job category than for an employer that has 500 employees in the same job category" (Department of Justice, n.d.). DOJ also states that absolute parity is not expected (Department of Justice, n.d.).

One possible cause of the differences between the percentage of an ethnic group in the county organization's workforce and the percentage of the ethnic group in the residential population may result from employees not necessarily residing within the same jurisdiction where they work. In Marin County, for example, 55\% of its workforce lives outside the county (Marin County, 2019e). This occurrence is exacerbated by the Bay Area's ongoing housing crisis (Plan Bay Area, 2019), which often forces employees to live outside the area where they work. This could be the reason why the City and County of San Francisco also considers data on migration patterns and public policies impact when creating their recruitment strategies.

Every county has its way of defining its relevant labor markets. Some counties, like Santa Clara, represent it based on the geographic origin of most of their applicants (County of Santa Clara, 2019b). For its 2019 utilization report, Santa Clara County used the weighted average of Santa Clara County, Alameda County, California State and United States as its baseline; however, in its 2017 report, it has only used the Santa Clara County labor market data (County of Santa Clara, 2019b). Marin County also shared in its 2020 EEO Plan that it used relevant labor market data that combines Sonoma, Alameda, Contra Costa, and Solano (Marin County, 2020). This research was not able to ascertain how the other counties decide what relevant labor market to use. Therefore, the labor market may differ from the residential population's ethnic diversity. 
The labor market statistics for Hispanics may be misleading regarding the numbers of qualified applicants in a population. For example, all people under 18 would have to be removed, since they should mostly still be in high school and not in the full-time job market. The Hispanic population is by far the youngest of the ethnicities in the Bay Area (Bay Area Equity Atlas, 2017).

Comparing the employee group with either the population or available labor market is a simple metric to seek information on diversity within a county government organization. Multiple other measures would be needed to determine a path toward equal representation in county government employment. Studies of applicant pool versus selected employees, longevity in county employment of current employees, and whether elements of the knowledge, skills, and abilities are inherently discriminatory would be critical in redesigning recruitment, job descriptions, and recruitment practices to achieve less disparity in employee ethnicities.

This study has provided a starting point to understand ethnic disparities in employee groups in county government employment in the Bay Area. Though this research has presented a case on the benefits of implementing diversity management programs, further study is needed to understand the overall employment practices and how they might be altered to enhance employment opportunities for a more equally representative group of employees. 


\section{CONCLUSION}

Though some county organizations in the Bay Area region have long expressed their awareness of the importance of workforce diversity, most counties are either at the beginning stages of their diversity management initiatives or still do not consider creating diversity policies a top priority.

Some jurisdictions have incorporated the strategies and best practices that IPMA has shared in the year 2000, but what is even more encouraging is the progress made over the last two decades, particularly with the greater attention given to the concepts of inclusion and equity. Organizations such as GARE seem to be at the forefront of driving these initiatives in the Bay Area.

Though not heavily emphasized in IPMA's list of best practices, county organizations such as Marin have also mentioned the inclusion of community members, particularly from underrepresented and disadvantaged communities, in developing their EEO and diversity plans, particularly in the conversation about equity. This conversation not only focuses on improving hiring practices but also on improving service delivery which is a testament to a bureaucracy's intent to move from a passive type of representation to an active one.

Although the Black or African American employee group is well represented across all nine Bay Area county organizations, the data in this research show that work is still needed when it comes to diversifying other ethnicities. Furthermore, the aspiration for representativeness should also manifest at the leadership level for these jurisdictions to become more effective in actively representing their constituents. Diversity management programs that standardize equity in every stage of the recruitment process, and inclusionary strategies that would boost morale and make every employee feel valued, could help attain this objective. 
Though most diversity management policies examined in this research have not been implemented long enough to be assessed for effectiveness soundly, future researchers could assess the long-term impact, internally and externally. Quantitative and qualitative data are now being gathered, as indicated in most of these plans. Future research could include a more complete analysis, such as how each of these programs compares with each other using variables like the population size of the jurisdiction, length of the diversity management program, types of strategies, and reporting structure (e.g., decentralization). Counties such as Marin, one of the first to create a robust diversity management plan, may serve as a case study on its effectiveness considering its low instance of underrepresentation. Conversely, research may show that restoring the policy of requiring racial quotas is needed to increase the representation of some ethnicities in county-level government employment. Many of the nine county organizations found disparities for Asian and Hispanic employee groups, particularly in leadership levels.

This research has also presented a high-level version of the labor market utilization report similar to what's being reported by the Equal Employment Opportunity (EEO) offices, focusing only on the employees' ethnicities overall and the employees in the Officials and Administrators category. Future research could consider other EEO job categories, such as Professionals, Technicians, Sworn Officers, Administrative Support, and Service Maintenance, to give a better picture of the dispersal of the different ethnic groups among the categories of county employment. Such a study might also cross-classify employees by gender and race (Department of Justice, n.d.). By doing so, EEO can easily spot hidden occurrences of underutilization. For example, though Hispanic employee groups may be overrepresented in Santa Clara County, a closer look suggests that most of them are overutilized in the Service Maintenance category but underutilized in middle management or leadership roles (Santa Clara, 2020). This research is 
merely a reflection of the racial and ethnic diversity of the Bay Area county governments' employees and not of other local governments (e.g., cities or special districts) or private organizations in the region.

Countless research and data sources have continuously proven the racial and ethnic diversification of the United States' population and its workforce. Workforce diversity is no longer just an optional strategy to attain organizational efficiency; it is now an American way of life. As Rice (2010) states, "we cannot avoid diversity, we face it and engage it everyday" (p. 55). And to stay efficient, attuned, and relevant in the eyes of its clientele, the public sector must learn to adapt. It has also been said that culture takes time to develop (Rice, 2010); therefore, public organizations must develop strategies that will prepare their workforce sooner rather than later to not fall victim to this change. Inclusion is more than just attaining demographic parity or representativeness; it is about creating a culture "that values members of the minority or outgroup in a way that enables those individuals to fully participate and view themselves as valued" (Feeney \& Camarena, 2021, p. 122).

This research may serve as an additional reference, not only for local government entities, but also for organizations exploring different strategies to promote and cultivate diversity within the workforce. It is also important to note that diversity management solutions may vary, depending on the need and current challenges of the organization (Rice, 2010). Additionally, although race is said to be the most important consideration in representative bureaucracy, other facets to diversity also warrant the same level of attention due to inequities, such as gender. If left unattended, diversity may come with many undesirable consequences and challenges, but it also comes with great opportunities if managed correctly. 


\section{REFERENCES}

Adejumo, V. (2021). Beyond diversity, inclusion, and belonging. Leadership (London, England), 17(1), 62-73. https://doi.org/10.1177/1742715020976202

Alameda County Training and Education Center. (n.d.). About Us. https://trainingcenter.acgov.org/about-us/

Alameda County. (2021a). Diversity Programs. http://www.acgov.org/diversity/

Alameda County. (2021b). Diversity Programs - What We Do.

http://www.acgov.org/diversity/whatwedo.htm

Alameda County. (2021c). Career Ladders. http://acgov.org/hrs/divisions/ps/ladders.htm

Alameda County. (2021d). Employee Assistance Program (EAP).

http://acgov.org/hrs/divisions/ebc/other_eap.htm

Alameda County. (2021e). Flexible Spending Accounts.

http://acgov.org/hrs/divisions/ebc/other_flexible.htm\#3

Alameda County. (2021f). Child Care Resources.

http://acgov.org/hrs/divisions/ebc/other_child.htm

Alameda County. (2021g). Commuter Benefits Program.

http://acgov.org/hrs/divisions/ebc/other_commuter.htm

Alameda County. (2021h). Catastrophic Sick Leave Program.

http://acgov.org/hrs/divisions/ebc/other_catastrophic.htm 
Ballotpedia. (2020). Federal and state affirmative action and anti-discrimination laws.

https://ballotpedia.org/Federal_and_state_affirmative_action_and_antidiscrimination_laws\#cite_note-BLRok-106

Bassett-Jones, N. (2005). The Paradox of Diversity Management, Creativity and Innovation. Creativity and Innovation Management, 14(2), 169-175. https://doi.org/10.1111/j.14678691.00337.x

Bay Area Equity Atlas. (2017). An Equity Profile of the Nine-County Bay Area Region [Data file].https://bayareaequityatlas.org/sites/default/files/Final_9_County_BayAreaProfile_0. pdf

Boden, S. (2020). Leading the way - Start Here: A Primer on Diversity and Inclusion (Part 1 of 2). Harvard Business Publishing. https://www.harvardbusiness.org/start-here-a-primer-on-diversity-and-inclusion-part-1of- $2 /$

California Legislative Information. (2002). Bill Analysis: SB 1045. California State Senate 8/24/02 Assembly Floor Analysis. https://leginfo.legislature.ca.gov/faces/billAnalysisClient.xhtml

City and County of San Francisco. (2012). Policy Regarding The Treatment of Co-Workers and Members of The Public [Data file]. https://sfdhr.org/sites/default/files/documents/DEI/Policy-Regarding-Treatment-ofCoworkers-and-Members-of-the-Public.pdf 
City and County of San Francisco. (2018). Executive Directive 18-02 [Data file].

https://sfdhr.org/sites/default/files/documents/Resources/Executive-Directive-18-02.pdf

City and County of San Francisco. (2019). Ordinance No. 188-19 - Office of Racial Equity [Data file]. https://sfgov.legistar.com/View.ashx?M=F\&ID=7586870\&GUID=9E0222B97A4D-4082-8CCE-3F397520FC82

City and County of San Francisco. (2020). Racial Equity Action Plan - December 2020 [Data file]. https://sfdhr.org/sites/default/files/documents/Reports/DHR-Racial-Equity-ActionPlan-2020.pdf

City and County of San Francisco. (n.d.-a). Managing Implicit Bias: Creating Awareness and Building Inclusion. https://sfdhr.org/managing-implicit-bias-creating-awareness-andbuilding-inclusion

City and County of San Francisco. (n.d.-b). Implicit Bias Training and Reporting for Department Heads and Commissioners. https://sfdhr.org/implicit-bias-training-and-reportingdepartment-heads-and-commissioners

City and County of San Francisco. (n.d.-c). Bridge to Success. https://sfdhr.org/apprenticeshipsfbridge-to-success

City and County of San Francisco. (n.d.-d). About Us. https://sfdhr.org/about-us

City and County of San Francisco. (n.d.-e). City University. https://sfdhr.org/city-university

City and County of San Francisco. (n.d.-f). SEIU Work Training Program. https://sfdhr.org/seiuwork-training-program 
City and County of San Francisco. (n.d.-g). 24-PLUS for Supervisors \& Managers. https://sfdhr.org/24-plus-supervisors-and-managers

City and County of San Francisco. (n.d.-h). Diversity, Equity, and Inclusion. https://sfdhr.org/node/57730

City and County of San Francisco. (n.d.-i). Pregnancy, Child Bonding, and Caregiving. https://sfdhr.org/pregnancy-child-bonding-and-caregiving

City and County of San Francisco. (n.d.-j). Workforce Development. https://sfdhr.org/workforcedevelopment-division

City and County of San Francisco. (n.d.-k). Race/Ethnicity and Classification. https://sfdhr.org/race-ethnicity-and-classification

Contra Costa County. (2017). Equal Employment Opportunity Outreach and Recruitment Plan [Data File]. https://www.contracosta.ca.gov/DocumentCenter/View/47289/Administrative-Bulletin143--Equal-Employment-Opportunity-Outreach-and-Recruitment-Plan?bidId=

Contra Costa County. (2018). Office of Equal Employment Opportunity FY 2017-2018 Outreach Report [Data

File].http://64.166.146.245/docs/2018/HOO/20181001_1198/35287_FY\%201819\%20H20\%20Report\%20FINAL.pdf

Contra Costa County. (2020a). Establishing a County Office of Racial Equity and Social Justice and Launching a Community Planning Process [Data File]. 
http://64.166.146.245/agenda_publish.cfm?id=\&mt=ALL\&get_month=11\&get_year=20 $20 \& \mathrm{dsp}=\mathrm{agm} \& \mathrm{seq}=43528 \& \mathrm{rev}=0 \& \mathrm{ag}=1592 \& \ln =87592 \& \mathrm{nseq}=43622 \&$ nrev $=0 \& \mathrm{pseq}=4$ 3674\&prev=0\#ReturnTo87592

Contra Costa County. (2020b). Racial Justice Oversight Body - Community Engagement \& Funding Subcommittee Meeting [Data File]. https://www.contracosta.ca.gov/DocumentCenter/View/62424/Racial-Justice-OversightBody-Community-Engagement-and-Funding-Subcommittee-Agenda-182020-PDF

Contra Costa County. (2021a). Hiring Outreach Oversight Committee. https://www.contracosta.ca.gov/4425/Hiring-Outreach-Oversight-Committee

Contra Costa County. (2021b). Employee Assistance Program (EAP). https://www.contracosta.ca.gov/1359/Employee-Assistance-Program

Contra Costa County. (2021c). Kids At Work Child Care Center. https://www.contracosta.ca.gov/3392/Kids-at-Work-Child-Care-Center

Contra Costa County. (2021d). Dependent Care Assistance Program. https://www.contracosta.ca.gov/1353/Dependent-Care-Assistance-Program

Contra Costa County. (2021e). Catastrophic Leave. https://www.contracosta.ca.gov/2251/Catastrophic-Leave

Contra Costa County. (2021f). Commuter Benefit Program. https://www.contracosta.ca.gov/6784/Commuter-Benefit-Program 
Cooper, C. A., \& Gerlach, J. D. (2019). Diversity Management in Action: Chief Diversity Officer Adoption in America's Cities. State \& Local Government Review, 51(2), 113121. https://doi.org/10.1177/0160323X19879735

County of San Mateo. (2017a). Accept and affirm leadership support for County Diversity and Inclusion Strategic Initiative. San Mateo County Board of Supervisors' Meeting, August 2017.

http://sanmateocounty.granicus.com/player/clip/113?view_id=1\&meta_id=22157\&redire ct $=$ true

County of San Mateo. (2017b). County of San Mateo Equal Employment Opportunity Plan [Data File]. https:/hr.smcgov.org/sites/hr.smcgov.org/files/EEO\%20Plan\%202018-2021.pdf

County of San Mateo. (2020). EEO Utilization Report [Data File]. https:/hr.smcgov.org/sites/hr.smcgov.org/files/documents/files/EEOP\%20Report\%20201 9\%20County-Final.do_.pdf

County of San Mateo. (2021). County of San Mateo Names First Equity Officer [Data File].https://www.smcgov.org/sites/smcgov.org/files/press-release/files/NEWS\%20SMC\%20Creates\%20New\%20Chief\%20Equity\%20Officer\%20Position_0.pdf

County of San Mateo. (n.d.-a). County of San Mateo Management Fellows Program [Data File]. https://hr.smcgov.org/sites/hr.smcgov.org/files/Management\%20Fellows2020\%20Guidelines\%20(SMC).pdf

County of San Mateo. (n.d.-b). EEO Advisory Committee. https://hr.smcgov.org/eeo-advisorycommittee 
County of San Mateo. (n.d.-c). Employee Assistance Program (EAP).

https://hr.smcgov.org/employee-assistance-program-eap

County of San Mateo. (n.d.-d). FAQs for Preventing Harassment in the Workplace Training. https://hr.smcgov.org/faqs-preventing-harassment-workplace-training

County of Santa Clara Employee Assistance Program. (2021). About Us. https://www.sccgov.org/sites/eap/About/Pages/About-EAP.aspx

County of Santa Clara Employee Services Agency. (2021a). Dependent Care Assistance Program (DCAP). https://employeeservices.sccgov.org/employee-benefits/life-andfinancial-benefits/dependent-care-assistance-program-dcap

County of Santa Clara Employee Services Agency. (2021b). Additional Benefits - VTA SmartPass. https://employeeservices.sccgov.org/employee-benefits/additional-benefits

County of Santa Clara Equal Opportunity Division. (2021). Training. https://equalopportunity.sccgov.org/training

County of Santa Clara. (1993). County of Santa Clara Policy on Diversity [Data File]. https://equalopportunity.sccgov.org/sites/g/files/exjcpb1126/files/policy-on-diversity.pdf

County of Santa Clara. (2017). Policy on Workforce Diversity - Board Policy 3.7 [Data File]. https://www.sccgov.org/sites/scc/gov/CountyPolicies/Board-Policy-3.7-Policy-onWorkforce-Diversity.pdf 
County of Santa Clara. (2019a). EEO Utilization Report [Data

File].https://equalopportunity.sccgov.org/sites/g/files/exjcpb1126/files/Documents/EEOP -shrt-frm2019-prt-1.pdf

County of Santa Clara. (2019b). Step 4b: EEOP Narrative of Interpretation - The County's

Workforce and Statistics [Data File].

https://equalopportunity.sccgov.org/sites/g/files/exjcpb1126/files/Documents/EEOP-prt2step-4b-2019.pdf

County of Santa Clara. (2020). Pay Equity Dashboard.

https://data.sccgov.org/stories/s/xrk2-3ryi

County of Santa Clara. (2021). Division of Equity and Social Justice.

https://desj.sccgov.org/home

County of Sonoma. (2020). Office of Equity Stablished. https://sonomacounty.ca.gov/EmployeeResources/News/Office-of-Equity-stablished/

County of Sonoma. (2021a). Sonoma County Five-Year Strategic Plan.

https://sonomacounty.ca.gov/Board-of-Supervisors/Strategic-Plan/

County of Sonoma. (2021b). Sonoma County Five-Year Strategic Plan [Data File].

https://sonomacounty.ca.gov/Board-of-Supervisors/Strategic-Plan/PDF/Five-YearStrategic-Plan-2021-26-English/ 
County of Sonoma. (2021c). Racial Equity and Social Justice.

https://sonomacounty.ca.gov/Board-of-Supervisors/Strategic-Plan/Racial-Equity-andSocial-Justice/

County of Sonoma. (2021d). Office of Equity: Meet our Team.

https://sonomacounty.ca.gov/Office-of-Equity/Meet-our-team/

County of Sonoma. (2021e). Workforce Development.

https://sonomacounty.ca.gov/HR/Workforce-Development/

County of Sonoma. (n.d.). Key Competencies \& Skills [Data File].

https://sonomacounty.ca.gov/WorkArea/DownloadAsset.aspx?id=2147521073

County of Sonoma. (2021f). Employee Assistance Program (EAP).

https://sonomacounty.ca.gov/HR/Benefits/Employee-Assistance-Program/

County of Sonoma. (2021g). SoCoLERN: Latinx Employee Resource Network. https://sonomacounty.ca.gov/SoCoLERN/

Crawford, R. (2020). 2020 Equal Employment Opportunity Plan. Marin County Board of Supervisors' Meeting, December 2020. https://marin.granicus.com/player/clip/10438?view_id=33\&redirect=true

Dennissen, M. H., Benschop, Y. W., \& Brink, M. C. L. (2020). Rethinking diversity management: An intersectional analysis of diversity networks. Organization Studies, 4l(2), 219-240. https://doi.org/10.1177/0170840618800103 
Feeney, M. K. \& Camarena, L. (2021). Gender, race, and diversity values among local government leaders. Review of Public Personnel Administration, 41(1), 105-131. https://journals.sagepub.com/doi/abs/10.1177/0734371X19865009

Foley, M. \& Williamson, S. (2019). Managerial Perspectives on Implicit Bias, Affirmative Action, and Merit. Public Administration Review, 79(1), 35-45. https://doi.org/10.1111/puar.12955

Geshekter, C. L. (2008). The Effects of Proposition 209 on California: Higher Education, Public Employment, and Contracting. Academic Questions, 21(3), 296-318. https://doi.org/10.1007/s12129-008-9072-8

Gonzales-Barrera, A. (2017). Recent trends in naturalization, 1995-2015. Pew Research. https://www.pewresearch.org/hispanic/2017/06/29/recent-trends-in-naturalization-1995$2015 /$

Gover, A. R., Harper, S. B., \& Langton, L. (2020). Anti-Asian hate crime during the COVID-19 pandemic: Exploring the reproduction of inequality. American Journal of Criminal Justice, 45(4), 647-667.

Government Alliance on Race \& Equity. (2021). Who We Are. https://www.racialequityalliance.org/about/who-we-are/

Holzer, H. \& Neumark, D. (1999). Are Affirmative Action Hires Less Qualified? Evidence from Employer-Employee Data on New Hires. Journal of Labor Economics, 17(3), 534-569. https://doi.org/10.1086/209930 
Hymel, M. (2020). Marin County Office of Equity Update. Marin County Board of Supervisors' Meeting, December 2020. https://marin.granicus.com/player/clip/10438?view_id=33\&redirect=true

ICMA. (n.d.). Social Equity: The Fourth Pillar of Public Administration. [Data File]. https://icma.org/sites/default/files/SOCIAL\%20EQUITY\%20THE\%20FOURTH\%20PILLAR\%20OF\%20PUBLIC\%20ADMINISTRATION\%20 Wooldridge $\% 20 \% 26 \% 20$ Bilharz.pdf

Ip, A. (2013). Trends and Reasons behind Asian Americans' Professional Choices. Presence Possibilities. https://ppossibilities.org/trends-and-reasons-behind-asian-americansprofessional-choices/

Klingner, D. E., Nalbandian, J. \& Llorens, J. (2010). Public Personnel Management (6th ed.). Pearson Education, Inc.

Koellen, T. (2021). Diversity management: A critical review and agenda for the future. Journal of Management Inquiry, 30(3), 259-272. https://journals.sagepub.com/doi/abs/10.1177/1056492619868025

Lewis, G. B. \& Yoon J. C. (2011). The Aging of the State Government Workforce: Trends and Implications. American Review of Public Administration, 41(1), 48-60. https://doi.org/10.1177/0275074009359308

Marin County. (2015a). County of Marin 5-Year Business Plan 2015-2020. https://www.marincounty.org/-/media/files/maringov/boardactions/2015/october/1510132cao5yearplanattach.pdf?la=en 
Marin County. (2015b, October 15). Supervisors Approve 5-Year Business Plan.

https://www.marincounty.org/main/county-press-releases/press-releases/2015/cao-5-yrplan-101315

Marin County. (2017a). Staff Report - 2017 Marin County Racial Equity Action Plan.https://www.marincounty.org/-/media/files/maringov/boardactions/2017/april/17042511 caoracialequityltr.pdf?la=en

Marin County. (2017b). County of Marin Racial Equity Action Plan 2017. https://www.marincounty.org//media/files/departments/hr/eeo/marin_racial_equity_action_plan2017.pdf?la=en

Marin County. (2017c). News Release-County Commits to Actions on Racial Equity. https://www.marincounty.org/main/county-press-releases/press-releases/2017/cao-equity042817

Marin County. (2018). News Release - Apply Now for Innovative Internship with County. https://www.marincounty.org/main/county-press-releases/press-releases/2018/probexplorers-042518

Marin County. (2019a). Department of Human Resource - Equity Dashboard. https://www.marincountyhr.org/get-to-know-us/diversity-and-inclusion/equitydashboard

Marin County. (2019b). Diversity Hiring Toolkit. https://www.marincountyhr.org/findemployee-tools/diversitytkmain 
Marin County. (2019c). Diversity and Inclusion. https://www.marincountyhr.org/get-to-knowus/diversity-and-inclusion

Marin County. (2019d). Employee Assistance Program. https://www.marincountyhr.org/learnabout-benefits/employee-assistance-program

Marin County. (2019e). Employee Housing Assistance Program. https://www.marincountyhr.org/learn-about-benefits/employee-housing-assistanceprogram

Marin County. (2020a). Diverse Volunteers Needed for Race Equity Planning Committee. https://www.marincounty.org/main/county-press-releases/press-releases/2020/caoequitycommittee-100720

Marin County. (2020b). County Prioritizes Racial Equity with Community. https://www.marincounty.org/main/county-press-releases/press-releases/2020/cao-equity120920

Marin County. (n.d.). Marin County Human Resources Department Career Ladders [Data file]. https://www.marincountyhr.org/-/media/files/departments/hr/employmentopportunities/career_ladders_cover_memo.pdf?la=en

Muse, A. (2020). Marin County Office of Equity Update. Marin County Board of Supervisors' Meeting, December 2020. https://marin.granicus.com/player/clip/10438?view_id=33\&redirect=true 
Myers, C. K. (2007). A Cure for Discrimination? Affirmative Action and the Case of California's Proposition 209. ILR Review, 60(3), 379-396. https://doiorg.libaccess.sjlibrary.org/10.1177/001979390706000304

Nakamura, D. (2021, August 30). Hate crimes rise to highest level in 12 years amid increasing attacks on Black and Asian people, FBI says. Washington Post. https://www.washingtonpost.com/national-security/hate-crimes-fbi-2020-asianblack/2021/08/30/28bede00-09a7-11ec-9781-07796ffb56fe_story.html

Napa County. (2021). Employee Benefits: Public Service Employee [Data File]. https://www.countyofnapa.org/DocumentCenter/View/7508/Benefits-Summary---PublicService-Employees-PSE

Nicholson, A. (2019). 5 Year Business Plan End of Year Four Report. Marin County Board of Supervisors' Meeting, December 2019. https://marin.granicus.com/player/clip/9788?view_id=33\&redirect=true

Office of Racial Equity. (n.d.). About the Office of Racial Equity. https://www.racialequitysf.org/about

Ohemeng, F. L. K. \& McGrandle, J. (2021). Employee perception of inclusivity and organizational performance: The case of the Ontario public service. Canadian Public Administration, 64(1), 26-50. https://doi.org/10.1111/capa.12407

Opie, T., \& Roberts, L. M. (2017). Do black lives really matter in the workplace? Restorative justice as a means to reclaim humanity. Equality, Diversity and Inclusion an International Journal, 36(8), 707-719. https://doi.org/10.1108/EDI-07-2017-0149 
Opportunities for All. (n.d.). Opportunities for All. https://www.opps4allsf.org/

Park, M. (2017). Accept and affirm leadership support for County Diversity and Inclusion Strategic Initiative. San Mateo County Board of Supervisors' Meeting, August 2017. http://sanmateocounty.granicus.com/player/clip/113?view_id=1\&meta_id=22157\&redire $\mathrm{ct}=$ true

Passel, J. S. \& D'Vera C. D. (2008). US population projections, 2005-2050. Pew Research Center. https://www.pewresearch.org/hispanic/2008/02/11/us-population-projections2005-2050/

Pitts, D. W., Hicklin, A. K., Hawes, D. P., \& Melton, E. (2010). What Drives the Implementation of Diversity Management Programs? Evidence from Public Organizations. Journal of Public Administration Research and Theory, 20(4), 867-886. https://doi.org/10.1093/jopart/mup044

Plan Bay Area 2040. (2019). The Bay Area today. http://2040.planbayarea.org/the-bay-areatoday

Porteshawver, A. (2019). 5 Year Business Plan End of Year Four Report.. Marin County Board of Supervisors' Meeting, December 2019. https://marin.granicus.com/player/clip/9788?view_id=33\&redirect=true

Rabl, T., del Carmen T. M., Byun, S.-Y., \& Bosch, L. (2020). Diversity Management Efforts as an Ethical Responsibility: How Employees' Perceptions of an Organizational Integration and Learning Approach to Diversity Affect Employee Behavior. Journal of Business Ethics, 161(3), 531-550. https://doi.org/10.1007/s10551-018-3849-7 
Reichenberg, N. E. (2001). Best Practices in Diversity Management. Cornell University Library. https://digitalcommons.ilr.cornell.edu/gladnetcollect/309/

Researchgate. (2008). The Nine-County San Francisco Bay Area. [Map]. Researchgate. https://www.researchgate.net/figure/The-nine-county-San-Francisco-BayArea_fig1_249994242

Riccucci, N. M. (2009). The Pursuit of Social Equity in the Federal Government: A Road Less Traveled? Public Administration Review, 69(3), 373-382. https://doi.org/10.1111/j.15406210.2009.01984.x

Riccucci, N. M. (2016). Public Personnel Management: Current Concerns, Future Challenges (5th ed.). Routledge.

Riccucci, N. M. \& Van Ryzin, G. G. (2017). Representative bureaucracy: a lever to enhance social equity, coproduction, and democracy. Public Administration Review, 77(1), 21-30. https://onlinelibrary.wiley.com/doi/abs/10.1111/puar.12649

Rice, L. \& Barth, J. M. (2017). A Tale of Two Gender Roles: The Effects of Implicit and Explicit Gender Role Traditionalism and Occupational Stereotype on Hiring Decisions. Gender Issues, 34(1), 86-102. https://doi.org/10.1007/s12147-016-9175-4

Rice, M.F. (2010). Diversity and Public Administration (2nd ed.). M.E. Sharpe, Inc.

San Francisco Health Service System. (2021). Employee Assistance Program. https://sfhss.org/eap 
Sandoval, T. (2018). How to Overcome Implicit Bias in Hiring. The Chronicle of Philanthropy, $30(9), 49$.

Santa Clara County Public Health. (2021). Racial and Health Equity. https://publichealth.sccgov.org/about-us/racial-and-health-equity

Saxena, A. (2014). Workforce Diversity: A Key to Improve Productivity. Procedia Economics and Finance, 11, 76-85. https://doi.org/10.1016/S2212-5671(14)00178-6

Schwartz, S. A. (2020). Police brutality and racism in America. Explore (New York, NY), 16(5), 280. https://www.ncbi.nlm.nih.gov/pmc/articles/PMC7331505/

Segal, J. (2017). The Risk of Bias Testing. https://www.shrm.org/hr-today/news/hr-magazine/0917/pages/the-risks-of-biastesting.aspx

Segrest Purkiss, S. L., Perrewé, P. L., Gillespie, T. L., Mayes, B. T., \& Ferris, G. R. (2006). Implicit sources of bias in employment interview judgments and decisions. Organizational Behavior and Human Decision Processes, 101(2), 152-167. https://doi.org/10.1016/j.obhdp.2006.06.005

Selden, S. C. \& Selden, F. (2001). Rethinking Diversity in Public Organizations for the 21st Century. Administration \& Society, 33(3), 303-329. https://doi.org/10.1177/00953990122019785 
SHRM (2019, October 25). Commuter Benefits Are an Investment in Employees.

https://www.shrm.org/resourcesandtools/hr-topics/benefits/pages/commuter-benefits-arean-investment-in-employees.aspx

Siddiqui, F., Hawkins, D., Shammas, B., \& Thebault, R. (2021, May 27). Authorities look for motive in San Jose transit shooting as community mourns 9 victims. Washington Post. https://www.washingtonpost.com/nation/2021/05/27/san-jose-shooting/

Simas, S. (2019). What is a Skelly Hearing? https://simasgovlaw.com/what-is-a-skelly-hearing/

Solano County. (n.d.). Other Benefits.

https://www.solanocounty.com/depts/hr/employeebenes/other.asp

Soni, V. (2000). A Twenty-First-Century Reception for Diversity in the Public Sector: A Case Study. Public Administration Review, 60(5), 395-408. https://doi.org/10.1111/00333352.00103

Sumner, M. D. (2011). Proposition 209 and Public Employment in California: Trends in Workforce Diversity. Berkeley Law. https://www.law.berkeley.edu/wpcontent/uploads/2016/07/Proposition-209-and-Public-Employment-WorkforceDiversity.pdf

Sylvia, R. \& Sylvia, K. (2012). Program Planning and Evaluation for the Public Manager. Waveland Press, Inc.

The White House. (2011). Executive Order 13583-- Establishing a Coordinated Governmentwide Initiative to Promote Diversity and Inclusion in the Federal Workforce. 
https://obamawhitehouse.archives.gov/the-press-office/2011/08/18/executive-order13583-establishing-coordinated-government-wide-initiativ

U.S. Bureau of Labor Statistics. (2015, December). Labor force projection to 2024: the labor force is growing but slowly. https://www.bls.gov/opub/mlr/2015/article/labor-forceprojections-to-2024.htm

U.S. Department of Justice. (n.d.). Office of Justice Programs: EEOP FAQs. https://www.ojp.gov/program/civil-rights/eeop/faqs\#faq-what-materials-are-needed-tocomplete-an-eeop

U.S. Equal Employment Opportunity Commission. (2011, August 8). President Signs Order To Promote Diversity And Inclusion In The Federal Workforce. https://www.eeoc.gov/newsroom/president-signs-order-promote-diversity-and-inclusionfederal-workforce

United States Census Bureau Quick Facts. (2019). Educational Attainment In the United States: 2019. https://www.census.gov/content/census/en/data/tables/2019/demo/educationalattainment/cps-detailed-tables.html

United States Census Bureau Quick Facts. (2021). Dashboard-United States. https://www.census.gov/quickfacts/fact/dashboard/US/PST045219

United States Census Bureau. (2020a, October 16). About. https://www.census.gov/topics/population/race/about.html 
United States Census Bureau. (2020b, October 16). About Hispanic Origin.

https://www.census.gov/topics/population/hispanic-origin/about.html

Vaillancourt, D. (2017). Accept and affirm leadership support for County Diversity and

Inclusion Strategic Initiative. Staff presentation, San Mateo County Board of Supervisors' Meeting, August 8, 2017.

http://sanmateocounty.granicus.com/player/clip/113?view_id=1\&meta_id=22157\&redire ct $=$ true

Valley Water. (2021). Diversity and Inclusion Program.

https://www.valleywater.org/how-we-operate/about-valley-water/diversity-and-inclusionprogram 